\title{
Heterotic-string amplitudes at one loop: modular graph forms and relations to open strings
}

\author{
Jan E. Gerken, ${ }^{a}$ Axel Kleinschmidt ${ }^{a, b}$ and Oliver Schlotterer ${ }^{a, c}$ \\ ${ }^{a}$ Max-Planck-Institut für Gravitationsphysik, Albert-Einstein-Institut, \\ 14476 Potsdam, Germany \\ ${ }^{b}$ International Solvay Institutes, \\ ULB-Campus Plaine CP231, BE-1050 Brussels, Belgium \\ ${ }^{c}$ Perimeter Institute for Theoretical Physics, \\ Waterloo, ON N2L 2Y5, Canada \\ E-mail: jan.gerken@aei.mpg.de, axel.kleinschmidt@aei.mpg.de, \\ olivers@aei.mpg.de
}

ABSTRACT: We investigate one-loop four-point scattering of non-abelian gauge bosons in heterotic string theory and identify new connections with the corresponding open-string amplitude. In the low-energy expansion of the heterotic-string amplitude, the integrals over torus punctures are systematically evaluated in terms of modular graph forms, certain non-holomorphic modular forms. For a specific torus integral, the modular graph forms in the low-energy expansion are related to the elliptic multiple zeta values from the analogous open-string integrations over cylinder boundaries. The detailed correspondence between these modular graph forms and elliptic multiple zeta values supports a recent proposal for an elliptic generalization of the single-valued map at genus zero.

Keywords: Scattering Amplitudes, Superstrings and Heterotic Strings, Supergravity Models

ARXIV EPRINT: 1811.02548 


\section{Contents}

1 Introduction 1

2 Basics of heterotic-string amplitudes 3

2.1 Kronecker-Eisenstein series and elliptic functions 3

2.2 One-loop gauge amplitudes of the heterotic string 5

$\begin{array}{lll}2.3 & \text { Spin-summed current correlators } & 7\end{array}$

2.4 The key integrals over torus punctures 9

3 Basics of modular graph forms $\quad 11$

$\begin{array}{ll}3.1 \text { Modular graph functions } & 11\end{array}$

$\begin{array}{lll}3.2 & \text { Modular graph forms } & 14\end{array}$

$\begin{array}{lll}3.2 .1 & \text { Relations among modular graph forms } & 16\end{array}$

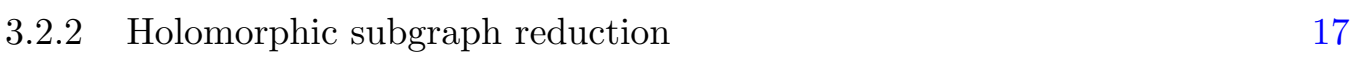

$\begin{array}{lll}3.2 .3 & \text { Trihedral modular graph forms } & 18\end{array}$

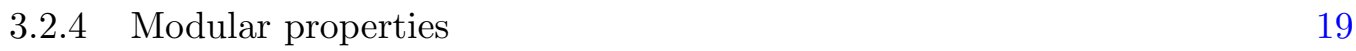

$\begin{array}{lll}3.3 & \text { Differential equations and iterated Eisenstein integrals } & 19\end{array}$

$\begin{array}{lll}\text { 3.3.1 Cauchy-Riemann derivatives of modular graph forms } & 19\end{array}$

$\begin{array}{lll}3.3 .2 & \text { Iterated Eisenstein integrals } & 20\end{array}$

$4 \alpha^{\prime}$-expansion of the heterotic-string amplitude and modular graph forms 23

$4.1 \alpha^{\prime}$-expansions from modular graph forms 23

4.1.1 Fourier expansion of the $V_{w}$ functions 24

4.1.2 Heterotic graph forms 24

$\begin{array}{ll}4.2 & \text { Planar heterotic graph forms } \\ \end{array}$

4.2.1 Leading orders $\alpha^{\prime 0}$ and $\alpha^{\prime 1} \quad 26$

$\begin{array}{ll}\text { 4.2.2 Subleading orders } \alpha^{\prime 2} \text { and } \alpha^{\prime 3} & 27\end{array}$

$\begin{array}{lll}\text { 4.2.3 Checking and assembling the results } & 28\end{array}$

$\begin{array}{lll}4.3 & \text { Non-planar heterotic graph forms } & 29\end{array}$

4.3.1 Leading orders $\alpha^{\prime 0}$ and $\alpha^{\prime 1} \quad 30$

4.3.2 Subleading orders $\alpha^{\prime 2}, \alpha^{3}$ and beyond 31

4.3.3 Assembling the results 32

4.4 Uniform transcendentality and $\alpha^{\prime}$-expansions 32

4.4.1 The planar results in terms of uniform-transcendentality integrals 34

4.4.2 The non-planar results in terms of uniform-transcendentality integrals 35

4.5 The integrated amplitude and the low-energy effective action 35

4.5.1 Planar amplitude up to second order in $\alpha^{\prime} \quad 36$

4.5.2 Non-planar amplitude up to second order in $\alpha^{\prime} \quad 36$

$\begin{array}{lll}\text { 4.5.3 Consistency with tree-level amplitudes } & 37\end{array}$

4.6 Modular graph forms in the massless $n$-point function 38

$\begin{array}{lll}4.6 .1 & n \text { external gauge bosons } & 38\end{array}$

$\begin{array}{lll}\text { 4.6.2 Adjoining external gravitons } & 39\end{array}$ 
$5 \quad$ Heterotic strings versus open superstrings

5.1 Review of the single-valued map in tree amplitudes $\quad 40$

5.2 Open-superstring integrals at genus one 41

5.2.1 Decomposition into symmetry components 42

5.2.2 Modular transformation $\quad 43$

5.3 A proposal for a single-valued map at genus one $\quad 45$

5.4 The closed-string integral over $V_{2}(1,2,3,4)$ versus esv $Z_{1234}^{(2)} \quad 46$

5.4.1 The closed-string expansion in terms of iterated Eisenstein integrals 47

5.4.2 The $P_{\text {Re }}$ projection 48

5.4.3 The relation between $Z_{1234}^{(2)}$ and $\mathcal{I}_{1234}^{(2,0)} \quad 49$

$\begin{array}{ll}5.4 .4 & \text { Integration cycles versus elliptic functions } \\ \end{array}$

6 Conclusions $\quad 51$

A Relations among modular graph forms $\quad \mathbf{5 2}$

A.1 Relations among dihedral graphs at weight $(4,0)$ and order $\alpha^{\prime 2} \quad 53$

A.2 Relations among dihedral graphs at weight $(2,0)$ and order $\alpha^{\prime 3} \quad 53$

A.3 Eisenstein regularized sums

$\begin{array}{lll}\text { A.4 Relations among trihedral graphs } & 55\end{array}$

A.5 Tetrahedral holomorphic subgraph reduction $\quad 56$

B The $V_{2}(1,2,3,4)$ integral at the third order in $\alpha^{\prime} \quad 57$

B.1 The twelve inequivalent heterotic graph forms $\quad 57$

B.2 Identities among modular graph forms at order $\alpha^{\prime 3} \quad 58$

C The $V_{2}(i, j)$ integrals at the third order in $\alpha^{\prime} \quad 58$

C.1 The inequivalent heterotic graph forms of weight $(2,0) \quad 58$

C.2 The inequivalent heterotic graph forms of weight $(4,0) \quad 59$

D Decomposition into uniform-transcendentality integrals $\quad 59$

$\begin{array}{lll}\text { D.1 Planar integrals } & 59\end{array}$

$\begin{array}{lll}\text { D.1.1 Integration-by-parts manipulations } & 60\end{array}$

D.1.2 Uniform transcendentality decomposition 61

D.1.3 Consistency check of the leading contributions to $\widehat{\mathcal{I}}_{1234}^{(4,0)} \quad 62$

$\begin{array}{lll}\text { D.2 Non-planar integrals } & 62\end{array}$

D.2.1 Rewriting the integral $\mathcal{I}_{12 \mid 34}^{(2,0)} \quad 63$

$\begin{array}{lll}\text { D.2.2 Rewriting the integral } \mathcal{I}_{12 \mid 34}^{(4,0)} & 64\end{array}$

D.2.3 Towards a uniform-transcendentality basis 64

D.3 Efficiency of the new representations for higher-order expansions 65

E The elliptic single-valued map at the third order in $\alpha^{\prime} \quad 65$

E.1 Modular transformation of iterated Eisenstein integrals $\quad 65$

E.2 The third $\alpha^{\prime}$-order of $\mathcal{I}_{1234}^{(2,0)}$ and iterated Eisenstein integrals 66 


\section{Introduction}

Scattering amplitudes in string theories have become a rewarding laboratory to encounter modern number-theoretic concepts in a simple setup. String amplitudes are derived from integrating over moduli spaces of punctured Riemann surfaces whose genus matches the loop order in perturbation theory. Accordingly, the low-energy expansion of string amplitudes provides generating functions for the periods of the relevant moduli spaces multiple zeta values at tree level [1-4] and various elliptic generalizations at loop level.

At genus one, a variety of mathematical structures have recently been revealed in maximally supersymmetric open- and closed-string amplitudes. For one-loop amplitudes of the open superstring, elliptic multiple zeta values (eMZVs) [5] were identified as the natural language to capture the expansion in the inverse string tension $\alpha^{\prime}[6,7]$. Closedstring amplitudes of type-II superstrings in turn introduce an intriguing system of nonholomorphic modular functions known as modular graph functions [8].

One-loop closed-string worldsheets are toroidal with complex modulus $\tau$ on which the modular group acts. Modular graph functions are invariant under this action and depend on a graph on the worldsheet describing the contractions of the punctures related to external states. The properties and relations of modular graph functions have been studied from various perspectives [9-22]. For open strings at genus one, eMZVs result from integration over the punctures on the boundary of a cylindrical open-string worldsheet at one-loop level, where $\tau$ is treated as the modulus of the cylinder.

This work is dedicated to one-loop amplitudes of the heterotic string, where the modulispace integrals are constrained by only half of the supersymmetries as compared to the type-II setup. Accordingly, the $\alpha^{\prime}$-expansion of heterotic-string amplitudes admits a larger class of integrals over torus punctures which we will evaluate in terms of modular graph forms in representative four-point examples. Modular graph forms have been introduced as generalizations of modular graph functions [15] by allowing a non-trivial transformation under the modular group action that is expressed through non-vanishing modular weights $(w, \bar{w})$. They can often be related to modular invariant functions by differential equations in $\tau$.

In this article, we will simplify the four-point amplitude among gauge bosons in a way such that the integrals over the punctures at any order of the $\alpha^{\prime}$-expansions manifestly evaluate to modular graph forms. Explicit results are given for both the single-trace and the double-trace sector up to the third subleading order in $\alpha^{\prime}$. Moreover, we give general arguments that modular graph forms capture the integrations over the torus punctures in the $n$-point heterotic-string amplitude involving any combination of gauge bosons and gravitons. Earlier work on the correlation functions and integrations over the punctures in multi-particle amplitudes of the heterotic string includes [23-25]. Furthermore, the subleading order in the $\alpha^{\prime}$-expansion of four-point amplitudes involving gravitons has been recently studied in [26, 27].

A major motivation for this work concerns the connection between open- and closedstring amplitudes at the level of their low-energy expansion. At genus zero, the multiple zeta values (MZVs) from open- and closed-string scattering on a disk and a sphere, re- 
spectively, are related by the single-valued map [3, 28-32]. ${ }^{1}$ The latter refers to the origin of MZVs from polylogarithms, where the defining property of single-valued MZVs is their descent from single-valued polylogarithms [33, 34]. The notion of a single-valued map applies to a variety of periods [35], and it is natural to expect loop-level relations between open- and closed strings on these grounds. An ambitious long-term goal is to completely sidestep the moduli-space integrations of closed-string amplitudes at various genera and to infer their results from suitable maps acting on open-string quantities.

In particular, by comparing the eMZVs and modular graph functions in one-loop amplitudes of open and closed strings, a conjecture for the explicit form of an elliptic singlevalued map has been made in [22]. This conjecture is based on a graphical organization of the open-string $\alpha^{\prime}$-expansion, but it has been limited to the scattering of abelian gauge bosons on the open-string side and modular invariant functions of $\tau$ on the closed-string side. As one of our main results, we extend the proposal of [22] to non-abelian open-string states, where the conjectural elliptic single-valued map reproduces substantial parts of modular graph forms of modular weight $(2,0)$. The integration cycles for four open-string punctures on a cylinder boundary are proposed to relate to certain elliptic functions of the closed-string punctures through a Betti-deRham duality [36, 37].

In the mathematics literature, a general construction of single-valued eMZVs has been given by Brown [38, 39]. So far, it remains conjectural that modular graph functions or modular graph forms are contained in the image of this elliptic single-valued map. The real-analytic functions in Brown's construction [38, 39] include modular forms of various holomorphic and antiholomorphic weights. Hence, our extension of the string-theory motivated conjecture for an elliptic single-valued map to modular graph forms should be helpful to identify the missing link to the setup of $[38,39]$. Given that the heterotic string relaxes the maximal supersymmetry of type-II superstrings, its extended set of moduli-space integrals (as investigated here at four points) is hoped to give a more general picture of an elliptic single-valued map.

As another consequence of the half-maximal supersymmetry of the heterotic string, the coefficients of a given modular graph form in massless one-loop ${ }^{2}$ amplitudes usually mix different orders in $\alpha^{\prime}$. In superstring amplitudes in turn, the order in the $\alpha^{\prime}$-expansion correlates with the transcendental weights of the accompanying iterated integrals - (elliptic) multiple zeta values or modular graph functions $[3,4,6,10]$. This property known as uniform transcendentality can also be found in the context of dimensionally regularized Feynman integrals with the regularization parameter $\varepsilon$ taking the rôle of $\alpha^{\prime}$ [42-46]. We decompose the four-point gauge amplitude of the heterotic string into integrals that are individually believed to be uniformly transcendental — both in the single-trace and the double-trace sector. The non-uniform transcendentality of the overall amplitude is then reflected by the coefficients of these basis integrals. The classification of uniformly transcendental moduli-space integrals is expected to give important clues about the mathematical properties of the underlying twisted cohomologies [47].

\footnotetext{
${ }^{1}$ The conjectures of $[3,28,29]$ have been proven recently in [30, 32]; see also [31] for a derivation where certain transcendentality conjectures on MZVs are assumed.

${ }^{2}$ See $[40,41]$ for the analogous phenomenon in tree-level amplitudes of the heterotic string, where the coefficients of a given multiple zeta value are usually geometric series in $\alpha^{\prime}$.
} 
In summary, the main results of this work are the following:

- a general argument and explicit four-point examples that modular graph forms capture the integrals over the punctures in massless one-loop amplitudes of the heterotic string

- relating an integral of modular weight $(2,0)$ in the four-point gauge amplitude of the heterotic string to a cylinder integral of the open superstring by applying the tentative elliptic single-valued map of [22] order by order in the $\alpha^{\prime}$-expansion

- an explicit decomposition of the four-point one-loop gauge amplitude of the heterotic string into integrals of conjecturally uniform transcendentality

This paper is structured as follows. We first review the worldsheet building blocks for constructing one-loop heterotic-string amplitudes in section 2. Then we develop the notion of modular graph forms and review their salient properties in section 3. In section 4, we bring the two concepts together and show how the integrands of one-loop amplitudes of the heterotic string can be expressed as modular graph forms. In particular, we explicitly perform the analysis of the leading planar and non-planar contributions to four-point gauge amplitudes in the $\alpha^{\prime}$-expansion. We also reorganize the integrals in terms of representatives of uniform transcendentality, determine the integrated amplitude to second order in $\alpha^{\prime}$ and comment on general $n$-point amplitudes and their relation to modular graph forms. In section 5, we investigate the relation between open-string amplitudes and heterotic strings and develop the conjectural notion of single-valued map relevant in this context. Section 6 contains concluding remarks. Several technical details needed in the analysis have been relegated to a number of appendices.

\section{Basics of heterotic-string amplitudes}

In this section, we introduce the basic building blocks for correlation functions in heteroticstring amplitudes at one loop and how they combine to produce modular forms after integration over the punctures corresponding to the external states.

\subsection{Kronecker-Eisenstein series and elliptic functions}

The relevant heterotic-string correlation functions are defined to live on a worldsheet of torus topology that will be parametrized by the parallelogram in figure 1 . The homology cycles of the torus are mapped to the periodicities $z \cong z+1$ and $z \cong z+\tau$ for the torus coordinate $z \in \mathbb{C}$, and the modular parameter $\tau \in \mathbb{C}$ is taken to be in the upper half plane $\operatorname{Im} \tau>0$.

In this setup, a universal starting point for constructing elliptic functions and iterated integrals is the following definition of the Kronecker-Eisenstein series [48, 49]

$$
F(z, \beta, \tau):=\frac{\theta_{1}^{\prime}(0, \tau) \theta_{1}(z+\beta, \tau)}{\theta_{1}(z, \tau) \theta_{1}(\beta, \tau)}
$$



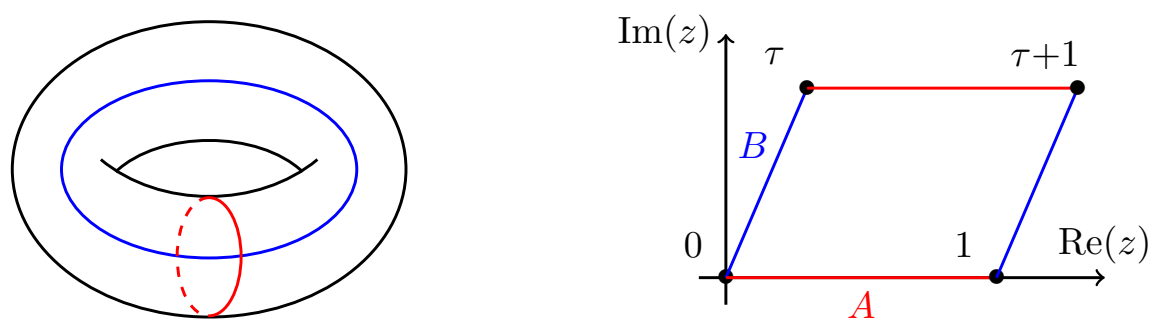

Figure 1. Throughout this work, toroidal genus-one worldsheets are parametrized through the depicted parallelogram with discrete identifications $z \cong z+1$ and $z \cong z+\tau$ corresponding to the $A$-cycle and $B$-cycle, respectively.

involving the odd Jacobi theta function

$$
\theta_{1}(z, \tau):=2 q^{1 / 8} \sin (\pi z) \prod_{n=1}^{\infty}\left(1-q^{n}\right)\left(1-e^{2 \pi i z} q^{n}\right)\left(1-e^{-2 \pi i z} q^{n}\right)
$$

and its $z$-derivative. The formal parameter $\beta$ appearing in definition (2.1) will be used below to define an infinite family of doubly-periodic functions. The Kronecker-Eisenstein series itself is quasi-periodic with [50]

$$
F(z+1, \beta, \tau)=F(z, \beta, \tau), \quad F(z+\tau, \beta, \tau)=e^{-2 \pi i \beta} F(z, \beta, \tau),
$$

but its monodromies around the $B$-cycle cancel from the following doubly-periodic completion

$$
\Omega(z, \beta, \tau):=\exp \left(2 \pi i \beta \frac{\operatorname{Im} z}{\operatorname{Im} \tau}\right) F(z, \beta, \tau) .
$$

When viewing $\beta$ as a bookkeeping variable, a Laurent expansion of (2.4) introduces doublyperiodic but non-meromorphic functions $f^{(w)}[6]$

$$
\Omega(z, \beta, \tau)=: \sum_{w=0}^{\infty} \beta^{w-1} f^{(w)}(z, \tau),
$$

starting with $f^{(0)}(z, \tau)=1$ and $f^{(1)}(z, \tau)=\partial_{z} \log \theta_{1}(z, \tau)+2 \pi i \frac{\operatorname{Im} z}{\operatorname{Im} \tau}$. From the KroneckerEisenstein series (2.1), one can see that the only singularity among the $f^{(w)}$ functions is the simple pole $f^{(1)}(z, \tau)=\frac{1}{z}+\mathcal{O}(z, \bar{z})$ at the origin (and its translates by $\mathbb{Z}+\tau \mathbb{Z}$ ).

The non-holomorphic exponentials of (2.4) drop out if several Kronecker-Eisenstein series are combined to cyclic products with first arguments $z_{i j}=z_{i}-z_{j}$ : a formal expansion in the bookkeeping variable $\beta$ defines a series of elliptic functions $V_{w}[25]^{3}$

$$
\begin{aligned}
F\left(z_{12}, \beta, \tau\right) F\left(z_{23}, \beta, \tau\right) \ldots F\left(z_{n 1}, \beta, \tau\right) & =\Omega\left(z_{12}, \beta, \tau\right) \Omega\left(z_{23}, \beta, \tau\right) \ldots \Omega\left(z_{n 1}, \beta, \tau\right) \\
& =: \beta^{-n} \sum_{w=0}^{\infty} \beta^{w} V_{w}(1,2, \ldots, n) .
\end{aligned}
$$

\footnotetext{
${ }^{3}$ Since $(2.6)$ is at the same time an elliptic function of $\beta$, the coefficients $V_{w}(1, \ldots, n), w<n$ of the singular terms in $\beta$ determine the coefficients $V_{w}(1, \ldots, n), w>n$ of the regular terms $\beta^{\geq 1}$ [25].
} 
Here and in later places of this work, the dependence of $V_{w}(1,2, \ldots, n)=V_{w}(1,2, \ldots, n \mid \tau)$ on the modular parameter is left implicit. The definition (2.6) and $F(-z,-\beta, \tau)=$ $-F(z, \beta, \tau)$ immediately manifest the following dihedral symmetry properties

$$
V_{w}(1,2, \ldots, n)=V_{w}(2, \ldots, n, 1), \quad V_{w}(n, n-1, \ldots, 2,1)=(-1)^{w} V_{w}(1,2, \ldots, n)
$$

under cyclic shifts and reversal. The $V_{w}$ functions can be conveniently expressed in terms of the doubly-periodic coefficients $f^{(w)}$ in (2.5), for instance (with cyclic identification $\left.z_{n+1}=z_{1}\right)$

$$
\begin{aligned}
& V_{0}(1,2, \ldots, n)=1, \quad V_{1}(1,2, \ldots, n)=\sum_{j=1}^{n} f^{(1)}\left(z_{j}-z_{j+1}, \tau\right) \\
& V_{2}(1,2, \ldots, n)=\sum_{j=1}^{n} f^{(2)}\left(z_{j}-z_{j+1}, \tau\right)+\sum_{i=1}^{n} \sum_{j=i+1}^{n} f^{(1)}\left(z_{i}-z_{i+1}, \tau\right) f^{(1)}\left(z_{j}-z_{j+1}, \tau\right) .
\end{aligned}
$$

The modular properties of the Kronecker-Eisenstein series (2.3) [50] give rise to the $\mathrm{SL}_{2}(\mathbb{Z})$ transformations

$$
\begin{gathered}
f^{(w)}\left(\frac{z}{\gamma \tau+\delta}, \frac{\alpha \tau+\beta}{\gamma \tau+\delta}\right)=(\gamma \tau+\delta)^{w} f^{(w)}(z, \tau) \\
\left.V_{w}(1,2, \ldots, n)\right|_{\tau \rightarrow \frac{\alpha \tau+\beta}{\gamma \tau+\delta}} ^{z_{j} \rightarrow \frac{z_{j}}{\gamma \tau+\delta}} \begin{array}{l}
(\gamma \tau+\delta)^{w} V_{w}(1,2, \ldots, n),
\end{array}
\end{gathered}
$$

where $\left(\begin{array}{cc}\alpha & \beta \\ \gamma & \delta\end{array}\right) \in \mathrm{SL}_{2}(\mathbb{Z})$. The purely holomorphic modular weight $(w, 0)$ of the $V_{w}$ functions will be later on seen to resonate with modular invariance of the heterotic string.

\subsection{One-loop gauge amplitudes of the heterotic string}

In this work, we will be interested in one-loop scattering of gauge bosons in the heterotic string. Up to an overall normalization factor, the prescription for the four-point function reads [51-54]

$$
\mathcal{M}_{4}=\int_{\mathcal{F}} \frac{\mathrm{d}^{2} \tau}{(\operatorname{Im} \tau)^{5}} \frac{1}{\eta^{24}(\tau)} \int_{T(\tau)} \mathrm{d}^{2} z_{2} \int_{T(\tau)} \mathrm{d}^{2} z_{3} \int_{T(\tau)} \mathrm{d}^{2} z_{4}\left\langle\prod_{j=1}^{4} \mathcal{V}^{a_{j}}\left(z_{j}, \epsilon_{j}, k_{j}\right)\right\rangle^{\tau},
$$

where $\mathcal{F}$ denotes the fundamental domain of the modular group $\mathrm{SL}_{2}(\mathbb{Z})$, the integration domain $T(\tau)$ for $z_{2}, z_{3}, z_{4}$ is the torus in the parametrization of figure 1 , and translation invariance has been used to fix $z_{1}=0$. Moreover, the inverse factors of the Dedekind eta function

$$
\eta(\tau):=q^{1 / 24} \prod_{n=1}^{\infty}\left(1-q^{n}\right), \quad q:=e^{2 \pi i \tau}
$$

arise as the partition function of the 26 worldsheet bosons in the non-supersymmetric sector, and $\mathcal{V}^{a}(z, \epsilon, k)$ denotes the vertex operator for an external gauge boson [51]

$$
\mathcal{V}^{a}(z, \epsilon, k)=J^{a}(z) V_{\mathrm{SUSY}}(\bar{z}, \epsilon, k) e^{i k \cdot X(z, \bar{z})}
$$


with polarization vector $\epsilon$, lightlike momentum $k$ and adjoint index $a$. The correlation function $\langle\ldots\rangle^{\tau}$ in (2.10) is evaluated on a torus of modular parameter $\tau$ and allows factoring out the contribution from the Kac-Moody currents $J^{a}(z)$. The leftover correlator involving $V_{\mathrm{SUSY}}(\bar{z}, \epsilon, k)$ and $e^{i k \cdot X(z, \bar{z})}$ matches a chiral half of type-II superstrings, and its four-point instance is completely determined by maximal supersymmetry [55],

$$
\left\langle\prod_{j=1}^{4} V_{\mathrm{SUSY}}\left(\bar{z}_{j}, \epsilon_{j}, k_{j}\right) e^{i k_{j} \cdot X\left(z_{j}, \bar{z}_{j}\right)}\right\rangle^{\tau}=\left(k_{1} \cdot k_{2}\right)\left(k_{2} \cdot k_{3}\right) A_{\mathrm{SYM}}^{\mathrm{tree}}(1,2,3,4)\left\langle\prod_{j=1}^{4} e^{i k_{j} \cdot X\left(z_{j}, \bar{z}_{j}\right)}\right\rangle^{\tau}
$$

The permutation invariant combination of polarizations $\epsilon$ and momenta $k$ on the righthand side has been expressed through a color-ordered tree-level amplitude $A_{\mathrm{SYM}}^{\text {tree }}(1,2,3,4)$ of ten-dimensional super-Yang-Mills. The manifestly supersymmetric calculation in the pure-spinor formalism [56] leads to the same conclusion for any combination of gauge bosons and gauginos. The left-over correlator over the plane waves

$$
\left\langle\prod_{j=1}^{n} e^{i k_{j} \cdot X\left(z_{j}, \bar{z}_{j}\right)}\right\rangle^{\tau}=\exp \left(\sum_{1 \leq i<j}^{n} s_{i j} G_{i j}(\tau)\right)
$$

will be referred to as the Koba-Nielsen factor and involves the bosonic Green function $G_{i j}(\tau):=G\left(z_{i}-z_{j}, \tau\right)$ as well as dimensionless Mandelstam invariants in the exponent,

$$
G(z, \tau):=-\log \left|\frac{\theta_{1}(z, \tau)}{\eta(\tau)}\right|^{2}-\frac{\pi(z-\bar{z})^{2}}{2 \operatorname{Im} \tau}, \quad s_{i j}:=-\frac{\alpha^{\prime}}{2} k_{i} \cdot k_{j}
$$

Note that this representation of the Green function relates it to the function $f^{(1)}(z, \tau)$ defined in (2.5) via

$$
f^{(1)}(z, \tau)=-\partial_{z} G(z, \tau)
$$

We shall now focus on the correlation function of the Kac-Moody currents $J^{a}$, see (2.10) and (2.12), that carries all the dependence on the adjoint indices $a_{1}, \ldots, a_{4}$ of the external gauge bosons. In a fermionic representation $J^{a}(z)=t_{i j}^{a} \psi^{i} \psi^{j}(z)$ of the currents, the correlators receive contributions from different spin structures - the boundary conditions for the worldsheet spinors under $\psi^{j}(z+1)= \pm \psi^{j}(z)$ and $\psi^{j}(z+\tau)= \pm \psi^{j}(z)$. We will be mostly interested in the gauge group $\operatorname{Spin}(32) / \mathbb{Z}_{2}$ with Lie-algebra generators $t_{i j}^{a}$ and fundamental indices $i, j=1,2, \ldots, 32$.

For four-point functions, the only contributions come from the even spin structures that we label with an integer $\nu=2,3,4$, and the corresponding fermionic two-point function or "Szegö kernel" [57] can be brought into the universal form

$$
S_{\nu}(z, \tau):=\frac{\theta_{1}^{\prime}(0, \tau) \theta_{\nu}(z, \tau)}{\theta_{\nu}(0, \tau) \theta_{1}(z, \tau)}
$$


where the even theta functions analogous to (2.2) read

$$
\begin{aligned}
& \theta_{2}(z, \tau):=2 q^{1 / 8} \cos (\pi z) \prod_{n=1}^{\infty}\left(1-q^{n}\right)\left(1+e^{2 \pi i z} q^{n}\right)\left(1+e^{-2 \pi i z} q^{n}\right) \\
& \theta_{3}(z, \tau):=\prod_{n=1}^{\infty}\left(1-q^{n}\right)\left(1+e^{2 \pi i z} q^{n-1 / 2}\right)\left(1+e^{-2 \pi i z} q^{n-1 / 2}\right) \\
& \theta_{4}(z, \tau):=\prod_{n=1}^{\infty}\left(1-q^{n}\right)\left(1-e^{2 \pi i z} q^{n-1 / 2}\right)\left(1-e^{-2 \pi i z} q^{n-1 / 2}\right)
\end{aligned}
$$

In the fermionic realization of Kac-Moody currents, the contribution of a given spin structure to the correlation function reduces to (sums of products of) Szegö kernels (2.17), e.g. [25]

$$
\begin{gathered}
\left\langle J^{a_{1}}\left(z_{1}\right) J^{a_{2}}\left(z_{2}\right)\right\rangle_{\nu}^{\tau}=\operatorname{Tr}\left(t^{a_{1}} t^{a_{2}}\right) S_{\nu}\left(z_{12}, \tau\right) S_{\nu}\left(z_{21}, \tau\right) \\
\left\langle J^{a_{1}}\left(z_{1}\right) J^{a_{2}}\left(z_{2}\right) J^{a_{3}}\left(z_{3}\right)\right\rangle_{\nu}^{\tau}=\stackrel{\leftrightarrow}{\operatorname{Tr}}\left(t^{a_{1}} t^{a_{2}} t^{a_{3}}\right) S_{\nu}\left(z_{12}, \tau\right) S_{\nu}\left(z_{23}, \tau\right) S_{\nu}\left(z_{31}, \tau\right) \\
\left\langle J^{a_{1}}\left(z_{1}\right) J^{a_{2}}\left(z_{2}\right) J^{a_{3}}\left(z_{3}\right) J^{a_{4}}\left(z_{4}\right)\right\rangle_{\nu}^{\tau}=\stackrel{\leftrightarrow}{\operatorname{Tr}}\left(t^{a_{1}} t^{a_{2}} t^{a_{3}} t^{a_{4}}\right) S_{\nu}\left(z_{12}, \tau\right) S_{\nu}\left(z_{23}, \tau\right) S_{\nu}\left(z_{34}, \tau\right) S_{\nu}\left(z_{41}, \tau\right) \\
+\operatorname{Tr}\left(t^{a_{1}} t^{a_{2}}\right) \operatorname{Tr}\left(t^{a_{3}} t^{a_{4}}\right) S_{\nu}\left(z_{12}, \tau\right) S_{\nu}\left(z_{21}, \tau\right) S_{\nu}\left(z_{34}, \tau\right) S_{\nu}\left(z_{43}, \tau\right)+\operatorname{cyc}(2,3,4),
\end{gathered}
$$

where we use the following shorthand for parity-weighted traces relevant for $n \geq 3$ currents,

$$
\stackrel{\leftrightarrow}{\operatorname{Tr}}\left(t^{a_{1}} t^{a_{2}} \ldots t^{a_{n}}\right):=\operatorname{Tr}\left(t^{a_{1}} t^{a_{2}} \ldots t^{a_{n}}\right)+(-1)^{n} \operatorname{Tr}\left(t^{a_{n}} \ldots t^{a_{2}} t^{a_{1}}\right)
$$

The sum over cyclic permutations refers to both lines of (2.21), and it acts on both the adjoint indices $a_{2}, a_{3}, a_{4}$ and the punctures $z_{2}, z_{3}, z_{4}$. Furthermore, each of the spinstructure dependent current correlators is weighted by the fermionic partition function of the $\operatorname{Spin}(32) / \mathbb{Z}_{2}$ model,

$$
Z_{\nu}^{\text {het }}(\tau):=2 \zeta_{4}^{2} \theta_{\nu}^{16}(0, \tau)
$$

Since we do not track the overall normalization of the amplitude in (2.10), the prefactor $2 \zeta_{4}^{2}$ is introduced along the way for later convenience. The end results for the current correlators in (2.10) are proportional to the spin-summed expressions,

$$
\left\langle J^{a_{1}}\left(z_{1}\right) J^{a_{2}}\left(z_{2}\right) \ldots J^{a_{n}}\left(z_{n}\right)\right\rangle^{\tau}=\sum_{\nu=2}^{4} Z_{\nu}^{\text {het }}(\tau)\left\langle J^{a_{1}}\left(z_{1}\right) J^{a_{2}}\left(z_{2}\right) \ldots J^{a_{n}}\left(z_{n}\right)\right\rangle_{\nu}^{\tau},
$$

and we will next construct convenient representations of (2.24) from the elliptic functions of section 2.1 .

\subsection{Spin-summed current correlators}

By the form of the spin-structure dependent correlators (2.19) and (2.21), we will decompose the spin sums (2.24) according to the traces of Lie-algebra generators. For $n$ gauge 
currents we get in general both single- and multi-trace contributions

$$
\begin{aligned}
\mathcal{H}_{12 \ldots n}(\tau):= & \left.\left\langle J^{a_{1}}\left(z_{1}\right) J^{a_{2}}\left(z_{2}\right) \ldots J^{a_{n}}\left(z_{n}\right)\right\rangle^{\tau}\right|_{\operatorname{Tr}\left(t^{a_{1}} t^{a_{2} \ldots} t^{a_{n-1}} t^{a_{n}}\right)} \\
= & \sum_{\nu=2}^{4} Z_{\nu}^{\text {het }}(\tau) S_{\nu}\left(z_{12}, \tau\right) S_{\nu}\left(z_{23}, \tau\right) \ldots S_{\nu}\left(z_{n-1, n}, \tau\right) S_{\nu}\left(z_{n 1}, \tau\right) \\
\mathcal{H}_{12 \ldots p \mid p+1 \ldots n}(\tau):= & \left.\left\langle J^{a_{1}}\left(z_{1}\right) J^{a_{2}}\left(z_{2}\right) \ldots J^{a_{n}}\left(z_{n}\right)\right\rangle^{\tau}\right|_{\operatorname{Tr}\left(t^{a_{1}} t^{a_{2} \ldots} t^{a_{p}}\right) \operatorname{Tr}\left(t^{a_{p+1} \ldots} t^{a_{n}}\right)} \\
= & \sum_{\nu=2}^{4} Z_{\nu}^{\text {het }}(\tau) S_{\nu}\left(z_{12}, \tau\right) S_{\nu}\left(z_{23}, \tau\right) \ldots S_{\nu}\left(z_{p-1, p}, \tau\right) S_{\nu}\left(z_{p 1}, \tau\right) \\
& \times S_{\nu}\left(z_{p+1, p+2}, \tau\right) S_{\nu}\left(z_{p+2, p+3}, \tau\right) \ldots S_{\nu}\left(z_{n-1, n}, \tau\right) S_{\nu}\left(z_{n, p+1}, \tau\right) .
\end{aligned}
$$

The dependence of the Szegö kernels (2.17) on the spin structure $\nu$ can be simplified by relating them to Kronecker-Eisenstein series (2.1) with one of the half-periods

$$
\omega_{2}=\frac{1}{2}, \quad \omega_{3}=-\frac{1+\tau}{2}, \quad \omega_{4}=\frac{\tau}{2}
$$

in the second argument $\beta$. Given that the $\theta_{\nu=1,2,3,4}$ functions can be mapped into each other by a half-period shift in the first argument, ${ }^{4}$ we have $S_{\nu}\left(z_{i j}, \tau\right) \sim F\left(z_{i j}, \omega_{\nu}, \tau\right)$ up to phase factors that drop out from the cyclic products of Szegö kernels in the spin sums (2.25) and $(2.26)[58]$

$$
S_{\nu}\left(z_{12}, \tau\right) S_{\nu}\left(z_{23}, \tau\right) \ldots S_{\nu}\left(z_{n 1}, \tau\right)=F\left(z_{12}, \omega_{\nu}, \tau\right) F\left(z_{23}, \omega_{\nu}, \tau\right) \ldots F\left(z_{n 1}, \omega_{\nu}, \tau\right)
$$

This naturally introduces the elliptic functions $V_{w}$ generated by the cycles of KroneckerEisenstein series in (2.6). Given that the right-hand side of (2.28) defines an elliptic function of $\omega_{\nu}$, all the spin-structure dependence can be absorbed into Weierstrass invariants

$$
e_{\nu}(\tau):=\wp\left(\omega_{\nu}, \tau\right), \quad \wp(z, \tau):=-\partial_{z}^{2} \log \theta_{1}(z, \tau)+\frac{\partial_{z}^{3} \theta_{1}(0, \tau)}{3 \partial_{z} \theta_{1}(0, \tau)} .
$$

The vanishing of $\partial_{z} \wp(z, \tau)$ at $z=\omega_{\nu}$ and the differential equation $\partial_{z}^{2} \wp(z, \tau)=6(\wp(z, \tau))^{2}-$ $30 \mathrm{G}_{4}$ then lead to a polynomial appearance of the Weierstrass invariants which carry the entire $\nu$-dependence $[58,59]$

$$
\begin{aligned}
S_{\nu}\left(z_{12}, \tau\right) S_{\nu}\left(z_{21}, \tau\right) & =V_{2}(1,2)+e_{\nu} \\
S_{\nu}\left(z_{12}, \tau\right) S_{\nu}\left(z_{23}, \tau\right) S_{\nu}\left(z_{31}, \tau\right) & =V_{3}(1,2,3)+e_{\nu} V_{1}(1,2,3) \\
S_{\nu}\left(z_{12}, \tau\right) S_{\nu}\left(z_{23}, \tau\right) S_{\nu}\left(z_{34}, \tau\right) S_{\nu}\left(z_{41}, \tau\right) & =V_{4}(1,2,3,4)+e_{\nu} V_{2}(1,2,3,4)+e_{\nu}^{2}-6 \mathrm{G}_{4} .
\end{aligned}
$$

\footnotetext{
${ }^{4}$ More explicitly, the theta functions defined in (2.2) and (2.18) are related by $\theta_{2}\left(z+\frac{1}{2}, \tau\right)=-\theta_{1}(z, \tau)$ and

$$
\theta_{4}\left(z+\frac{\tau}{2}, \tau\right)=i e^{-i \pi z} q^{-1 / 8} \theta_{1}(z, \tau), \quad \theta_{4}\left(z+\frac{1}{2}, \tau\right)=\theta_{3}(z, \tau), \quad \theta_{3}\left(z+\frac{\tau}{2}, \tau\right)=e^{-i \pi z} q^{-1 / 8} \theta_{2}(z, \tau) .
$$
}


We are using the following normalization conventions for holomorphic Eisenstein series, ${ }^{5}$

$$
\mathrm{G}_{k}(\tau):=\sum_{(m, n) \neq(0,0)} \frac{1}{(m \tau+n)^{k}}=2 \zeta_{k}+\frac{2(2 \pi i)^{k}}{(k-1) !} \sum_{m, n=1}^{\infty} m^{k-1} q^{m n}, \quad k \geq 4 \text { and even }
$$

where $(m, n) \neq(0,0)$ is a shorthand for the summation domain $(m, n) \in \mathbb{Z}^{2} \backslash\{(0,0)\}$. Since the Weierstrass invariants are furthermore related by $e_{\nu}^{3}-15 \mathrm{G}_{4} e_{\nu}-35 \mathrm{G}_{6}=0$, the spin sums of the $n$-point current correlators in (2.25) and (2.26) can be reduced to the three inequivalent cases $^{6}[53]$

$$
\sum_{\nu=2}^{4} Z_{\nu}^{\text {het }}=\mathrm{G}_{4}^{2}, \quad \sum_{\nu=2}^{4} Z_{\nu}^{\text {het }} e_{\nu}=-\frac{7}{2} \mathrm{G}_{4} \mathrm{G}_{6}, \quad \sum_{\nu=2}^{4} Z_{\nu}^{\text {het }} e_{\nu}^{2}=\frac{49}{6} \mathrm{G}_{6}^{2}+\frac{5}{3} \mathrm{G}_{4}^{3} .
$$

As a bottom line, (2.30) and (2.32) lead us to the following representations

$$
\begin{aligned}
\mathcal{H}_{12} & =\mathrm{G}_{4}^{2} V_{2}(1,2)-\frac{7}{2} \mathrm{G}_{4} \mathrm{G}_{6} \\
\mathcal{H}_{123} & =\mathrm{G}_{4}^{2} V_{3}(1,2,3)-\frac{7}{2} \mathrm{G}_{4} \mathrm{G}_{6} V_{1}(1,2,3) \\
\mathcal{H}_{1234} & =\mathrm{G}_{4}^{2} V_{4}(1,2,3,4)-\frac{7}{2} \mathrm{G}_{4} \mathrm{G}_{6} V_{2}(1,2,3,4)-\frac{13}{3} \mathrm{G}_{4}^{3}+\frac{49}{6} \mathrm{G}_{6}^{2} \\
\mathcal{H}_{12 \mid 34} & =\mathrm{G}_{4}^{2} V_{2}(1,2) V_{2}(3,4)-\frac{7}{2} \mathrm{G}_{4} \mathrm{G}_{6}\left[V_{2}(1,2)+V_{2}(3,4)\right]+\frac{5}{3} \mathrm{G}_{4}^{3}+\frac{49}{6} \mathrm{G}_{6}^{2}
\end{aligned}
$$

for the spin sums in (2.25) and (2.26) which enter the trace decomposition of the four-point correlator,

$$
\begin{aligned}
\left\langle J^{a_{1}}\left(z_{1}\right) J^{a_{2}}\left(z_{2}\right) J^{a_{3}}\left(z_{3}\right) J^{a_{4}}\left(z_{4}\right)\right\rangle^{\tau}=\stackrel{\leftrightarrow}{\operatorname{Tr}}\left(t^{a_{1}} t^{a_{2}} t^{a_{3}} t^{a_{4}}\right) \mathcal{H}_{1234} \\
+\operatorname{Tr}\left(t^{a_{1}} t^{a_{2}}\right) \operatorname{Tr}\left(t^{a_{3}} t^{a_{4}}\right) \mathcal{H}_{12 \mid 34}+\operatorname{cyc}(2,3,4) .
\end{aligned}
$$

As before, the sum over cyclic permutations refers to both lines of (2.37).

\subsection{The key integrals over torus punctures}

We shall now pinpoint the integrals over torus punctures that need to be performed in the four-point gauge amplitude (2.10) and whose low-energy expansion will be the main topic of the later sections. After factoring out the polarization dependent parts $(2.13),{ }^{7}$

$$
\mathcal{M}_{4}=\left(k_{1} \cdot k_{2}\right)\left(k_{2} \cdot k_{3}\right) A_{\mathrm{SYM}}^{\text {tree }}(1,2,3,4) \int_{\mathcal{F}} \frac{\mathrm{d}^{2} \tau}{(\operatorname{Im} \tau)^{2} \eta^{24}(\tau)} M_{4}(\tau),
$$

\footnotetext{
${ }^{5}$ Note that our normalization conventions in the expression (2.31) for holomorphic Eisenstein series $\mathrm{G}_{2 m}$ differ from (3.11) in [15] by a factor of $\pi^{m}$. The style of the letter $\mathrm{G}_{k}$ and the number of subscripts distinguishes the holomorphic Eisenstein series $\mathrm{G}_{k}$ in (2.31) from the bosonic Green functions $G_{i j}(\tau)=$ $G\left(z_{i}-z_{j}, \tau\right)$ in $(2.15)$.

${ }^{6}$ These identities are a consequence of the relations $e_{2}+e_{3}+e_{4}=0, e_{2} e_{3}+e_{3} e_{4}+e_{4} e_{2}=-15 \mathrm{G}_{4}$ and $e_{2} e_{3} e_{4}=35 \mathrm{G}_{6}$ among the Weierstrass invariants as well as the connection with theta functions via $\pi^{2}\left(\theta_{4}(0, \tau)\right)^{4}=e_{2}-e_{3}, \pi^{2}\left(\theta_{2}(0, \tau)\right)^{4}=e_{3}-e_{4}$ and $\pi^{2}\left(\theta_{3}(0, \tau)\right)^{4}=e_{2}-e_{4}$.

${ }^{7}$ In order to reproduce the normalization conventions for the four-point gauge amplitude in [53], the right-hand side of (2.38) needs to be multiplied by $\frac{1}{\left(2 \zeta_{4}\right)^{2}}\left(\frac{\alpha^{\prime} g}{64 \pi^{5}}\right)^{2}$, where $g$ denotes the gauge coupling. The inverse factor of $2 \zeta_{4}^{2}$ compensates for our choice of normalization of the partition function in (2.23).
} 
we will be interested in the following integral over the punctures,

$$
M_{4}(\tau)=\int \mathrm{d} \mu_{4}\left\langle J^{a_{1}}(0) J^{a_{2}}\left(z_{2}\right) J^{a_{3}}\left(z_{3}\right) J^{a_{4}}\left(z_{4}\right)\right\rangle^{\tau} \exp \left(\sum_{1 \leq i<j}^{4} s_{i j} G_{i j}(\tau)\right) .
$$

We use the following notation for the modular invariant integration measure,

$$
\int \mathrm{d} \mu_{4}:=\prod_{j=2}^{4} \int_{T(\tau)} \frac{\mathrm{d}^{2} z_{j}}{\operatorname{Im} \tau}
$$

and have used translation invariance on the torus to fix the first coordinate $z_{1} \rightarrow 0$ such that the integral is only over the remaining three punctures. The dimensionless Mandelstam invariants $s_{i j}$ obey the following constraints by momentum conservation and the mass-shell condition $k_{j}^{2}=0$ for external gauge bosons $j=1,2,3,4$,

$$
s_{34}=s_{12}, \quad s_{14}=s_{23}, \quad s_{13}=s_{24}=-s_{12}-s_{23} .
$$

With the results for the current correlators (2.37) in terms of the spin sums (2.35) and (2.36), the right-hand side of (2.39) boils down to five inequivalent Koba-Nielsen integrals $\mathcal{I}^{(w, 0)}$ over elliptic functions,

$$
\begin{aligned}
& M_{4}(\tau)=\stackrel{\leftrightarrow}{\operatorname{Tr}}\left(t^{a_{1}} t^{a_{2}} t^{a_{3}} t^{a_{4}}\right)\left[\mathrm{G}_{4}^{2} \mathcal{I}_{1234}^{(4,0)}-\frac{7}{2} \mathrm{G}_{4} \mathrm{G}_{6} \mathcal{I}_{1234}^{(2,0)}-\frac{13}{3} \mathrm{G}_{4}^{3} \mathcal{I}^{(0,0)}+\frac{49}{6} \mathrm{G}_{6}^{2} \mathcal{I}^{(0,0)}\right] \\
& +\operatorname{Tr}\left(t^{a_{1}} t^{a_{2}}\right) \operatorname{Tr}\left(t^{a_{3}} t^{a_{4}}\right)\left[\mathrm{G}_{4}^{2} \mathcal{I}_{12 \mid 34}^{(4,0)}-\frac{7}{2} \mathrm{G}_{4} \mathrm{G}_{6} \mathcal{I}_{12 \mid 34}^{(2,0)}+\frac{5}{3} \mathrm{G}_{4}^{3} \mathcal{I}^{(0,0)}+\frac{49}{6} \mathrm{G}_{6}^{2} \mathcal{I}^{(0,0)}\right]+\operatorname{cyc}(2,3,4) .
\end{aligned}
$$

The notation ' $+\operatorname{cyc}(2,3,4)$ ' refers to cyclic permutations of both lines w.r.t. the adjoint indices $a_{2}, a_{3}, a_{4}$ and the Mandelstam invariants $s_{i j}$. Two of the integrals in the single-trace or 'planar' sector of (2.42) are defined by a cyclic ordering in the subscript

$$
\begin{aligned}
& \mathcal{I}_{1234}^{(4,0)}\left(s_{i j}, \tau\right):=\int \mathrm{d} \mu_{4} V_{4}(1,2,3,4) \exp \left(\sum_{1 \leq i<j}^{4} s_{i j} G_{i j}(\tau)\right) \\
& \mathcal{I}_{1234}^{(2,0)}\left(s_{i j}, \tau\right):=\int \mathrm{d} \mu_{4} V_{2}(1,2,3,4) \exp \left(\sum_{1 \leq i<j}^{4} s_{i j} G_{i j}(\tau)\right) .
\end{aligned}
$$

Furthermore, the permutation-invariant integral

$$
\mathcal{I}^{(0,0)}\left(s_{i j}, \tau\right):=\int \mathrm{d} \mu_{4} \exp \left(\sum_{1 \leq i<j}^{4} s_{i j} G_{i j}(\tau)\right)
$$

is universal to the single- and double-trace sectors of (2.42), and it furthermore occurs in the four-point one-loop amplitude of type-II superstrings [55]. In the double-trace or 
'non-planar' sector of (2.42), we have further instances of $\mathcal{I}^{(0,0)}$ and

$$
\begin{aligned}
& \mathcal{I}_{12 \mid 34}^{(4,0)}\left(s_{i j}, \tau\right):=\int \mathrm{d} \mu_{4} V_{2}(1,2) V_{2}(3,4) \exp \left(\sum_{1 \leq i<j}^{4} s_{i j} G_{i j}(\tau)\right) \\
& \mathcal{I}_{12 \mid 34}^{(2,0)}\left(s_{i j}, \tau\right):=\int \mathrm{d} \mu_{4}\left[V_{2}(1,2)+V_{2}(3,4)\right] \exp \left(\sum_{1 \leq i<j}^{4} s_{i j} G_{i j}(\tau)\right) .
\end{aligned}
$$

In all of (2.43) to (2.47), translation invariance has been used to fix $z_{1}=0$.

The superscripts in the notation for the integrals keep track of their modular weights: by the modular properties (2.9) of the elliptic $V_{w}$-functions, the integrals are easily checked to transform as modular forms of holomorphic weights $(w, 0)$,

$$
\mathcal{I}_{\ldots}^{(w, 0)}\left(s_{i j}, \frac{\alpha \tau+\beta}{\gamma \tau+\delta}\right)=(\gamma \tau+\delta)^{w} \mathcal{I}_{\ldots}^{(w, 0)}\left(s_{i j}, \tau\right),
$$

where the ellipsis may represent any permutation of 1234 and 12|34 (or be empty to incorporate modular invariance of $\left.\mathcal{I}^{(0,0)}\right)$. With the modular weight $(k, 0)$ of $\mathrm{G}_{k}$, each term in the four-point integral $(2.42)$ is a form of weight $(12,0)$. In the integrated amplitude (2.38), this compensates the weight $(-12,0)$ of the bosonic partition function $\eta^{-24}$ in agreement with modular invariance.

In the rest of this work, we will develop and apply methods for a systematic lowenergy expansion of the integrals (2.43) to (2.47) over the punctures. This amounts to a simultaneous Taylor expansion in all the Mandelstam invariants (2.15) and will also be referred to as $\alpha^{\prime}$-expansion. The modular weights (2.48) apply to each order in the $\alpha^{\prime}$-expansion of the integrals $\mathcal{I}^{(w, 0)}$. For all of $(2.43)$ to $(2.47)$, the coefficients of any monomial in $s_{i j}$ will be shown to line up with modular graph forms [15] which we will review in the next section. Hence, the $\mathcal{I}^{(w, 0)}$ are generating series for modular graph forms of weight $(w, 0)$, and the modular invariants obtained from $\mathcal{I}^{(0,0)}$ are known as modular graph functions [8].

\section{Basics of modular graph forms}

In this section, we introduce some basic material and notation for modular graph functions (that are invariant under the modular group) and modular graph forms (that transform covariantly under the modular group).

\subsection{Modular graph functions}

One of the main goals in this paper is to perform the integrals over the punctures $z_{2}, z_{3}, z_{4}$ in the low-energy expansion of the $\tau$-integrand (2.42) of the heterotic-string amplitude (2.38). We will start with a review of the techniques that apply to the simplest integral $\mathcal{I}^{(0,0)}\left(s_{i j}, \tau\right)$ in (2.45) known from the type-II superstring [55]. While a closed-form evaluation of $\mathcal{I}^{(0,0)}\left(s_{i j}, \tau\right)$ is currently out of reach, its Taylor expansion in the $s_{i j}$ has been thoroughly investigated in $[8-11,20]$. As explained in the references, the $\alpha^{\prime}$-expansion of $\mathcal{I}^{(0,0)}\left(s_{i j}, \tau\right)$ probes low-energy effective interactions at the one-loop order of type-II superstrings and has important implications for S-duality of the type-IIB theory [60-64]. 


$$
G_{i j}(\tau) \leftrightarrow i \bullet \cdot j, \quad \int \mathrm{d} \mu_{4} G_{23} G_{24} G_{34}^{2} \leftrightarrow \searrow_{1}^{3}
$$

Figure 2. Graphical representation of a bosonic Green function $G_{i j}(\tau)$ and a sample integral in the low-energy expansion of $\mathcal{I}^{(0,0)}$.

However, by keeping $\tau$ fixed in this calculation, one cannot detect the logarithmic dependence of the full amplitude on $s_{i j}$ which arises from the $\tau \rightarrow i \infty$ region of the later integration over modular parameters, cf. (2.38). As explained in [10], Taylor expansion of (2.45) amounts to isolating the analytic momentum dependence of the one-loop amplitude as opposed to non-analytic threshold contributions including factors of log $s_{i j}$ that are determined from the tree amplitude via unitarity. The analytic momentum dependence of graviton and gauge-boson amplitudes in turn can be used to identify curvature and fieldstrength operators in the low-energy effective action. Still, a subtle interplay between the analytic and non-analytic sectors has to be taken into account $[10,11]$.

After Taylor expanding the exponentials in the integrand of (2.45), the leftover challenge is to integrate monomials in Green functions $\prod_{1 \leq i<j}^{4} G_{i j}^{n_{i j}}$ with $n_{i j} \in \mathbb{N}_{0}$ over the torus. This kind of order-by-order integration is most conveniently performed by means of the lattice-sum representation [9]

$$
G(z, \tau)=\frac{\operatorname{Im} \tau}{\pi} \sum_{(m, n) \neq(0,0)} \frac{e^{2 \pi i(m v-n u)}}{|m \tau+n|^{2}}, \quad z=u \tau+v,
$$

where $(m, n) \neq(0,0)$ is again a shorthand for $(m, n) \in \mathbb{Z}^{2} \backslash\{(0,0)\}$, and we use the following real coordinates $u, v \in[0,1)$ for the torus puncture $z=u \tau+v$ in (3.1) and later equations,

$$
u=\frac{\operatorname{Im} z}{\operatorname{Im} \tau}, \quad v=\operatorname{Re} z-\frac{\operatorname{Im} z \operatorname{Re} \tau}{\operatorname{Im} \tau} .
$$

Note that the lattice-sum representation (3.1) of the Green function requires a summation prescription as the sum is not absolutely convergent. We shall use the Eisenstein summation prescription defined in (A.4). The lattice sum exhibits the modular invariance of the Green function, i.e. the integral $\mathcal{I}^{(0,0)}\left(s_{i j}, \tau\right)$ is modular invariant term by term in its $\alpha^{\prime}$-expansion.

By the absence of the zero mode $(m, n)=(0,0)$ in $(3.1)$, a single Green function integrates to zero under the measure $\mathrm{d} \mu_{4}$ in (2.40). A large class of integrals over $\prod_{1 \leq i<j}^{4} G_{i j}^{n_{i j}}$ vanishes for the same reason, and the conditions on the exponents $n_{i j} \in \mathbb{N}_{0}$ can be characterized by the following graphical organization: assign a vertex for each puncture $z_{j}, j=1,2,3,4$ and draw an undirected edge between vertices $i$ and $j$ for each factor of $G_{i j}=G_{j i}$ in the integrand, see figure 2. The contributions from disconnected graphs factorize in this setup, and trivial graphs with just one zero-valent vertex evaluate to one by $\int_{T(\tau)} \frac{\mathrm{d}^{2} z}{\operatorname{Im} \tau}=1$. Furthermore, a monomial in $G_{i j}$ integrates to zero whenever the associated graph is one-particle reducible, i.e. whenever it can be disconnected by cutting a single line. 


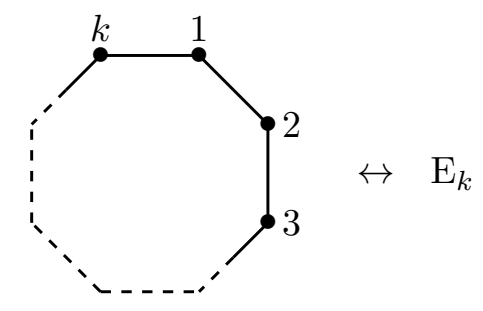

Figure 3. One-loop graphs due to a length- $k$ cycle of Green functions $G_{12} G_{23} \ldots G_{k-1, k} G_{k 1}$ correspond to non-holomorphic Eisenstein series $\mathrm{E}_{k}$ defined in (3.3).

The simplest non-vanishing integrals admitted by this graphical selection rule turn out to be non-holomorphic Eisenstein series

$$
\mathrm{E}_{k}(\tau):=\left(\frac{\operatorname{Im} \tau}{\pi}\right)^{k} \sum_{(m, n) \neq(0,0)} \frac{1}{|m \tau+n|^{2 k}}, \quad k \geq 2 .
$$

One can check from the lattice-sum representation (3.1) of the Green functions that closed cycles $G_{12} G_{23} \ldots G_{k-1, k} G_{k, 1}$ associated with one-loop graphs as depicted in figure 3 integrate to $\mathrm{E}_{k}(\tau)$, e.g.

$$
\int \mathrm{d} \mu_{4} G_{12}^{2}=\mathrm{E}_{2}, \quad \int \mathrm{d} \mu_{4} G_{12} G_{23} G_{31}=\mathrm{E}_{3}, \quad \int \mathrm{d} \mu_{4} G_{12} G_{23} G_{34} G_{41}=\mathrm{E}_{4} .
$$

In the graphical organization of the $\alpha^{\prime}$-expansion of $\mathcal{I}^{(0,0)}\left(s_{i j}, \tau\right)$, the denominators in (3.3) can be interpreted as Feynman propagators of a scalar field on a torus. The discrete momenta $m \tau+n$ arise from the Fourier coefficients of the $G_{i j}$ associated with the edges, see (3.1), and integration over the $z_{j}$ imposes momentum conservation at each vertex. The results of integrating more general monomials $\prod_{1 \leq i<j}^{N} G_{i j}^{n_{i j}}$ over the measure $\prod_{j=2}^{N} \frac{\mathrm{d}^{2} z_{j}}{\operatorname{Im} \tau}$ for $N$ punctures are dubbed modular graph functions [8]. Modular invariance of modular graph functions is a direct consequence of the modular invariance of the integration measure and the Green function.

Modular graph functions as the ones depicted in figure 3 with one loop on the toroidal worldsheet involve only a single lattice sum. Modular graph functions with more than one loop on the toroidal worldsheet as e.g. the ones depicted in figure 4 involve several lattice sums. In order to avoid cluttering notation, we shall use the shorthand $\sum_{p \neq 0}$ for the lattice sum $\sum_{(m, n) \neq(0,0)}$ over the discrete momenta $p=m \tau+n$ (instead of writing out $p \in(\mathbb{Z} \tau+\mathbb{Z}) \backslash\{0\})$. In this way, the expression (3.3) for one-loop graph functions condenses to $\mathrm{E}_{k}=\left(\frac{\operatorname{Im} \tau}{\pi}\right)^{k} \sum_{p \neq 0} \frac{1}{|p|^{2 k}}$, and the two-loop graphs depicted in figure 4 give rise to double lattice sums ${ }^{8}$

$$
C_{a, b, c}(\tau):=\left(\frac{\operatorname{Im} \tau}{\pi}\right)^{a+b+c} \sum_{\substack{p_{1}, p_{2} \neq 0 \\ p_{1}+p_{2} \neq 0}} \frac{1}{\left|p_{1}\right|^{2 a}\left|p_{2}\right|^{2 b}\left|p_{1}+p_{2}\right|^{2 c}} .
$$

\footnotetext{
${ }^{8}$ Without the notation for discrete momenta $p_{i}=m_{i} \tau+n_{i},(3.5)$ takes the lengthier form $C_{a, b, c}(\tau)=\left(\frac{\operatorname{Im} \tau}{\pi}\right)^{a+b+c} \sum_{\substack{\left(m_{1}, n_{1}\right),\left(m_{2}, n_{2}\right) \neq(0,0) \\\left(m_{1}, n_{1}\right)+\left(m_{2}, n_{2}\right) \neq(0,0)}} \frac{1}{\left|m_{1} \tau+n_{1}\right|^{2 a}\left|m_{2} \tau+n_{2}\right|^{2 b}\left|\left(m_{1}+m_{2}\right) \tau+\left(n_{1}+n_{2}\right)\right|^{2 c}}$.
} 


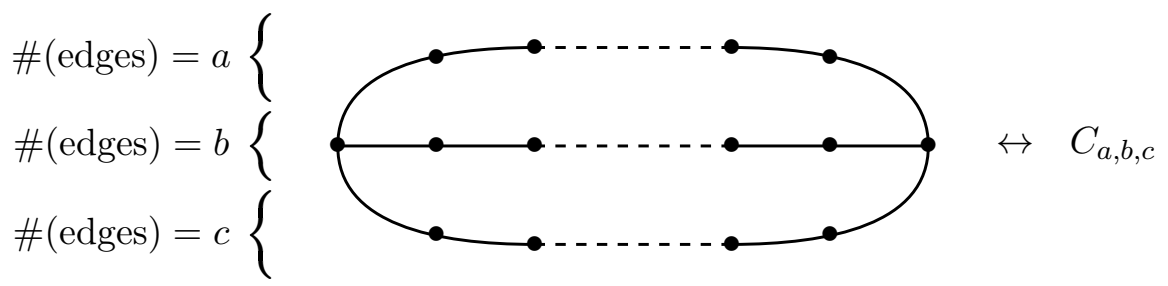

Figure 4. Two loop graphs associated with the modular graph functions $C_{a, b, c}$ defined in (3.5).

The modular invariant functions $C_{a, b, c}$ can be viewed as a generalization of non-holomorphic Eisenstein series, and many of their properties including Laplace eigenvalue equations and Fourier zero modes w.r.t. $\operatorname{Re} \tau$ have been studied for generic $a, b, c \geq 1[11,21]$. Their simplest examples in the $\alpha^{\prime}$-expansion of the integral (2.45) are

$$
\int \mathrm{d} \mu_{4} G_{12}^{3}=C_{1,1,1}, \quad \int \mathrm{d} \mu_{4} G_{12}^{2} G_{23} G_{31}=C_{2,1,1} .
$$

One of the fascinating properties of modular graph functions is their multitude of relations which often involve multiple zeta values (MZVs) and mix different loop orders $[11,12,19]$. For instance, the simplest two- and three-loop modular graph functions turn out to reduce to lower-complexity objects $[11,12]$

$$
C_{1,1,1}=\mathrm{E}_{3}+\zeta_{3}, \quad D_{4}:=\int \mathrm{d} \mu_{4} G_{12}^{4}=24 C_{2,1,1}-18 \mathrm{E}_{4}+3 \mathrm{E}_{2}^{2} .
$$

In general MZVs $\zeta_{n_{1}, n_{2}, \ldots, n_{r}}$ are defined by the conical sums

$$
\zeta_{n_{1}, n_{2}, \ldots, n_{r}}:=\sum_{0<k_{1}<k_{2}<\ldots<k_{r}} k_{1}^{-n_{1}} k_{2}^{-n_{2}} \ldots k_{r}^{-n_{r}}, \quad n_{i} \in \mathbb{N}, \quad n_{r} \geq 2,
$$

where $r$ and $n_{1}+n_{2}+\ldots+n_{r}$ are referred to as their depth and weight, respectively.

With the above input, the leading orders in the $\alpha^{\prime}$-expansion of the integral $(2.45)$ $\operatorname{read}^{9}$

$$
\begin{aligned}
\mathcal{I}^{(0,0)}\left(s_{i j}, \tau\right)= & 1+2 \mathrm{E}_{2}\left(s_{13}^{2}-s_{12} s_{23}\right)+\left(5 \mathrm{E}_{3}+\zeta_{3}\right) s_{12} s_{23} s_{13} \\
& +2\left(2 C_{2,1,1}+\mathrm{E}_{2}^{2}-\mathrm{E}_{4}\right)\left(s_{13}^{2}-s_{12} s_{23}\right)^{2}+\mathcal{O}\left(\alpha^{\prime 5}\right)
\end{aligned}
$$

and we will later on give similar expansions for the additional integrals that enter the heterotic-string amplitude (2.42).

\subsection{Modular graph forms}

The lattice-sum representations of holomorphic Eisenstein series $\mathrm{G}_{k}$ in (2.31) and their nonholomorphic counterparts $\mathrm{E}_{k}$ in (3.3) can be unified in the framework of modular graph

\footnotetext{
${ }^{9}$ Note that the relation $s_{12}+s_{13}+s_{23}=0$ among the Mandelstam variables in (3.9) has been used to attain compact expressions in the $\alpha^{\prime}$-expansion. Elimination of $s_{13}$ leaves $\mathcal{I}^{(0,0)}$ to be function of $s_{12}, s_{23}$ and $\tau$.
} 


$$
\sum_{p \neq 0} \frac{1}{p^{a} \bar{p}^{b}} \leftrightarrow \bullet(a, b) \longrightarrow, \quad \mathcal{C}\left[\begin{array}{llll}
a_{1} & a_{2} & \ldots & a_{R} \\
b_{1} & b_{2} & \ldots & b_{R}
\end{array}\right] \leftrightarrow \leftrightarrow
$$

Figure 5. Graph associated to a single lattice sum (left panel) and the general dihedral modular graph form $\mathcal{C}\left[\begin{array}{llll}a_{1} & a_{2} & \ldots & a_{R} \\ b_{1} & b_{2} & \ldots & b_{R}\end{array}\right]$ (right panel). The holomorphic and antiholomorphic decorations of the edges are denoted by $\left(a_{j}, b_{j}\right)$. The arrows indicate the directions of the momenta whose relative orientation is important for the momentum-conserving delta function.

forms [15]. The idea is to study lattice sums of the type $\sum_{p \neq 0} \frac{1}{p^{a} \bar{p}^{b}}$, where the holomorphic and antiholomorphic momenta $p=m \tau+n$ and $\bar{p}=m \bar{\tau}+n$ may have different integer powers $a \neq b$ with $a+b \geq 2$. Generalizations to multiple sums $\sum_{p_{1}, p_{2}, \ldots \neq 0}$ arise naturally from the following dictionary to one-particle irreducible graphs: associate powers of momenta $p^{-a} \bar{p}^{-b}$ with an edge decorated by the two integers $(a, b)$ as depicted in figure 5 and take vertices to impose momentum conservation as before. Under a change of direction $p \rightarrow-p$, the term $p^{-a} \bar{p}^{-b}$ changes sign if $a+b$ is odd, hence odd values of $a+b$ give rise to directed edges, while even values of $a+b$ give rise to undirected edges. Flipping the direction of a directed edge flips the sign of the associated modular graph form. It will be shown in section 4.1 that the $\alpha^{\prime}$-expansions of the integrals $\mathcal{I}_{\ldots}^{(w, 0)}$ defined in $(2.43)$ to $(2.47)$ are expressible in terms of modular graph forms.

For the dihedral graphs with two vertices and $R$ decorated edges $\left(a_{1}, b_{1}\right),\left(a_{2}, b_{2}\right), \ldots$, $\left(a_{R}, b_{R}\right)$ depicted in figure 5 , the associated modular graph form is

$$
\mathcal{C}\left[\begin{array}{cccc}
a_{1} & a_{2} & \ldots & a_{R} \\
b_{1} & b_{2} & \ldots & b_{R}
\end{array}\right]:=\left(\frac{\operatorname{Im} \tau}{\pi}\right)^{\sum_{i=1}^{R} b_{i}} \sum_{p_{1}, p_{2}, \ldots, p_{R} \neq 0} \frac{\delta\left(p_{1}+p_{2}+\ldots+p_{R}\right)}{p_{1}^{a_{1}} \bar{p}_{1}^{b_{1}} p_{2}^{a_{2}} \bar{p}_{2}^{b_{2}} \ldots p_{R}^{a_{R}} \bar{p}_{R}^{b_{R}}}
$$

which matches the notation of [15] up to overall powers of $\pi$ and $\operatorname{Im} \tau$. The normalization conventions in (3.10) with holomorphic and antiholomorphic momenta entering on asymmetric footing are chosen for later convenience. ${ }^{10}$ The momentum-conserving delta function is introduced to manifest the invariance of (3.10) under permutations $\sigma \in S_{R}$ of the edges and their decorations $\left(a_{i}, b_{i}\right)$,

$$
\mathcal{C}\left[\begin{array}{llll}
a_{1} & a_{2} & \ldots & a_{R} \\
b_{1} & b_{2} & \ldots & b_{R}
\end{array}\right]=\mathcal{C}\left[\begin{array}{cccc}
a_{\sigma(1)} & a_{\sigma(2)} & \ldots & a_{\sigma(R)} \\
b_{\sigma(1)} & b_{\sigma(2)} & \ldots & b_{\sigma(R)}
\end{array}\right] \quad \forall \sigma \in S_{R}
$$

After resolving the delta functions, the remaining summation variables $p_{j} \neq 0$ in

$$
\mathcal{C}\left[\begin{array}{ccc}
a_{1} & \ldots & a_{R} \\
b_{1} & \ldots & b_{R}
\end{array}\right]=\left(\frac{\operatorname{Im} \tau}{\pi}\right)^{\sum_{i=1}^{R} b_{i}} \sum_{\substack{p_{1}, \ldots, p_{R-1} \neq 0 \\
p_{1}+\ldots+p_{R-1} \neq 0}} \frac{(-1)^{a_{R}+b_{R}}}{p_{1}^{a_{1}} \bar{p}_{1}^{b_{1}} \ldots p_{R-1}^{a_{R-1}} \bar{p}_{R-1}^{b_{R-1}}\left(p_{1}+\ldots+p_{R-1}\right)^{a_{R}}\left(\bar{p}_{1}+\ldots+\bar{p}_{R-1}\right)^{b_{R}}}
$$

\footnotetext{
${ }^{10}$ The quantities which are denoted by $\mathcal{C}\left[\begin{array}{cccc}a_{1} & a_{2} & \ldots & a_{R} \\ b_{1} & b_{2} & \ldots & b_{R}\end{array}\right], \mathcal{C}^{+}\left[\begin{array}{cccc}a_{1} & a_{2} & \ldots & a_{R} \\ b_{1} & b_{2} & \ldots & b_{R}\end{array}\right]$ and $\mathcal{C}^{-}\left[\begin{array}{cccc}a_{1} & a_{2} & \ldots & a_{R} \\ b_{1} & b_{2} & \ldots & b_{R}\end{array}\right]$ in $[15]$ are obtained by multiplying (3.10) by factors of $\left(\frac{\operatorname{Im} \tau}{\pi}\right)^{\frac{1}{2}(a-b)},\left(\frac{(\operatorname{Im} \tau)^{2}}{\pi}\right)^{\frac{1}{2}(a-b)}$ and $\left(\frac{1}{\pi}\right)^{\frac{1}{2}(a-b)}$, respectively, where $a=\sum_{i=1}^{R} a_{i}$ and $b=\sum_{i=1}^{R} b_{i}$.
} 
are additionally constrained by $p_{1}+p_{2}+\ldots+p_{R-1} \neq 0$, and similar expressions are obtained upon eliminating a different momentum. Hence, a single summation variable is attained from (3.12) by formally starting with a two-edge graph,

$$
\mathcal{C}\left[\begin{array}{ll}
a & 0 \\
b & 0
\end{array}\right]=\left(\frac{\operatorname{Im} \tau}{\pi}\right)^{b} \sum_{p \neq 0} \frac{1}{p^{a} \bar{p}^{b}}
$$

and the two classes of Eisenstein series $\mathrm{G}_{k}$ and $\mathrm{E}_{k}$ are recovered via

$$
\mathrm{G}_{k}=\mathcal{C}\left[\begin{array}{ll}
k & 0 \\
0 & 0
\end{array}\right], \quad \mathrm{E}_{k}=\mathcal{C}\left[\begin{array}{cc}
k & 0 \\
k & 0
\end{array}\right] .
$$

Note that we require $a_{i}+b_{i}+a_{R}+b_{R}>2$ for all $i=1, \ldots, R-1$ in order to ensure absolute convergence of the series. ${ }^{11}$ If $a_{i}+b_{i}+a_{R}+b_{R}=2$ for some $i$, the sum is only conditionally convergent, as e.g. in the case of $G_{2}$. We define $G_{2}$ using the Eisenstein summation prescription $\sum_{\mathrm{E}}$ (cf. appendix A.3 and in particular (A.4)),

$$
\mathrm{G}_{2}(\tau):=\sum_{p \neq 0} \frac{1}{p^{2}}=\sum_{n \in \mathbb{Z} \backslash\{0\}} \frac{1}{n^{2}}+\sum_{m \in \mathbb{Z} \backslash\{0\}} \sum_{n \in \mathbb{Z}} \frac{1}{(m \tau+n)^{2}},
$$

which gives rise to a holomorphic but non-modular expression. One can also obtain a modular but non-holomorphic version $\hat{\mathrm{G}}_{2}$ via [65]

$$
\hat{\mathrm{G}}_{2}(\tau):=\lim _{s \rightarrow 0} \sum_{(m, n) \neq(0,0)} \frac{1}{(m \tau+n)^{2}|m \tau+n|^{s}}=\mathrm{G}_{2}(\tau)-\frac{\pi}{\operatorname{Im} \tau} .
$$

For some of these conditionally convergent lattice sums, regularized values $\mathcal{C}_{\mathrm{reg}}\left[\begin{array}{llll}a_{1} & a_{2} & \ldots & a_{R} \\ b_{1} & b_{2} & \ldots & b_{R}\end{array}\right]$ will be given in section 4.3 .

By definition, modular graph functions are special cases of modular graph forms, where the edges have decorations of the form $(a, a)$, i.e. where the holomorphic and antiholomorphic momenta always have the same exponents. For instance, the two-loop graph function in (3.5) and the three-loop graph function in (3.7) line up as follows with the notation of (3.12):

$$
C_{a, b, c}=\mathcal{C}\left[\begin{array}{lll}
a & b & c \\
a & b & c
\end{array}\right], \quad D_{4}=\mathcal{C}\left[\begin{array}{llll}
1 & 1 & 1 & 1 \\
1 & 1 & 1 & 1
\end{array}\right]
$$

\subsubsection{Relations among modular graph forms}

The momentum-conserving delta function in (3.10) gives rise to a variety of algebraic relations among absolutely convergent modular graph forms [15]. By inserting the vanishing momenta $\sum_{j=1}^{R} p_{j}$ or $\sum_{j=1}^{R} \bar{p}_{j}$ into the numerators for dihedral graphs, one finds

$$
0=\sum_{j=1}^{R} \mathcal{C}\left[\begin{array}{cccccc}
a_{1} & a_{2} & \ldots & a_{j}-1 & \ldots & a_{R} \\
b_{1} & b_{2} & \ldots & b_{j} & \ldots & b_{R}
\end{array}\right]=\sum_{j=1}^{R} \mathcal{C}\left[\begin{array}{cccccc}
a_{1} & a_{2} & \ldots & a_{j} & \ldots & a_{R} \\
b_{1} & b_{2} & \ldots & b_{j}-1 & \ldots & b_{R}
\end{array}\right] .
$$

\footnotetext{
${ }^{11}$ Since the columns in the matrix $\left(\begin{array}{ccccc}a_{1} & a_{2} & \ldots & a_{R} \\ b_{1} & b_{2} & \ldots & b_{R}\end{array}\right)$ can be rearranged arbitrarily by (3.11), this is equivalent to the criterion that $a_{i}+b_{i}+a_{j}+b_{j}>2$ for any $i, j=1,2, \ldots, R$.
} 
Whenever an edge in a dihedral graph carries the trivial decoration $\left(a_{j}, b_{j}\right)=(0,0)$, one can reduce the number of summation variables via

$$
\mathcal{C}\left[\begin{array}{lllll}
a_{1} & a_{2} & \ldots & a_{R-1} & 0 \\
b_{1} & b_{2} & \ldots & b_{R-1} & 0
\end{array}\right]=\prod_{j=1}^{R-1} \mathcal{C}\left[\begin{array}{ll}
a_{j} & 0 \\
b_{j} & 0
\end{array}\right]-\mathcal{C}\left[\begin{array}{llll}
a_{1} & a_{2} & \ldots & a_{R-1} \\
b_{1} & b_{2} & \ldots & b_{R-1}
\end{array}\right]
$$

where the last term stems from the constraint $\sum_{j=1}^{R-1} p_{j} \neq 0$ on the left-hand side. With $R=3$ edges, one can use the $R=2$ identity $\mathcal{C}\left[\begin{array}{ll}a & c \\ b & d\end{array}\right]=(-1)^{c+d} \mathcal{C}\left[\begin{array}{ll}a+c & 0 \\ b+d & 0\end{array}\right]$ to reduce the right-hand side to the simplest lattice sums (3.13),

$$
\mathcal{C}\left[\begin{array}{lll}
a & c & 0 \\
b & d & 0
\end{array}\right]=\mathcal{C}\left[\begin{array}{ll}
a & 0 \\
b & 0
\end{array}\right] \mathcal{C}\left[\begin{array}{ll}
c & 0 \\
d & 0
\end{array}\right]-(-1)^{c+d} \mathcal{C}\left[\begin{array}{lll}
a+c & 0 \\
b+d & 0
\end{array}\right] .
$$

By combinations of (3.18) and (3.19), one can often reduce the number of summation variables as in

$$
\mathcal{C}\left[\begin{array}{lll}
2 & 1 & 1 \\
0 & 1 & 1
\end{array}\right]=\mathcal{C}\left[\begin{array}{ll}
4 & 0 \\
2 & 0
\end{array}\right], \quad \mathcal{C}\left[\begin{array}{lll}
2 & 1 & 1 \\
1 & 1 & 0
\end{array}\right]=-\frac{1}{2} \mathcal{C}\left[\begin{array}{ll}
4 & 0 \\
2 & 0
\end{array}\right],
$$

and a variety of similar identities is spelled out in appendix A.1.

\subsubsection{Holomorphic subgraph reduction}

Another opportunity to reduce the number of summation variables in a modular graph form arises for graphs that have closed subgraphs of only holomorphic momenta [15, 66]. By the methods of [15], in the dihedral case, such "holomorphic subgraphs" can be expressed in terms of subgraphs without closed loops with coefficients $\mathrm{G}_{k \geq 4}$ or $\hat{\mathrm{G}}_{2}$. Given collective labels $A=\left(a_{1}, a_{2}, \ldots, a_{R-2}\right)$ and $B=\left(b_{1}, b_{2}, \ldots, b_{R-2}\right)$ for the decorations and $a_{+}+a_{-} \geq 3$, we have

$$
\begin{aligned}
& \mathcal{C}\left[\begin{array}{ccc}
a_{+} & a_{-} & A \\
0 & 0 & B
\end{array}\right]=\mathcal{C}\left[\begin{array}{cc}
a_{+} & a_{-} \\
0 & 0
\end{array}\right] \mathcal{C}\left[\begin{array}{c}
A \\
B
\end{array}\right]-\left(\begin{array}{c}
a_{+}+a_{-} \\
a_{-}
\end{array}\right) \mathcal{C}\left[\begin{array}{cc}
a_{+}+a_{-} & A \\
0 & B
\end{array}\right]
\end{aligned}
$$

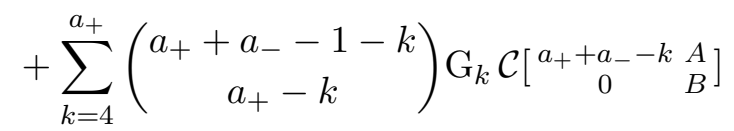

$$
\begin{aligned}
& +\sum_{k=4}^{a_{-}}\left(\begin{array}{c}
a_{+}+a_{-}-1-k \\
a_{-}-k
\end{array}\right) \mathrm{G}_{k} \mathcal{C}\left[\begin{array}{cc}
a_{+}+a_{-}-k & A \\
0 & B
\end{array}\right] \\
& +\left(\begin{array}{c}
a_{+}+a_{-}-2 \\
a_{+}-1
\end{array}\right)\left\{\hat{\mathrm{G}}_{2} \mathcal{C}\left[\begin{array}{cc}
a_{+}+a_{-}-2 & A \\
0 & B
\end{array}\right]+\mathcal{C}\left[\begin{array}{cc}
a_{+}+a_{-}-1 & A \\
-1 & B
\end{array}\right]\right\}
\end{aligned}
$$

where the entry -1 of the last term can be resolved via momentum conservation (3.18). As detailed in [15] and reviewed in appendix A.3, the contributions of $\hat{\mathrm{G}}_{2}$ or $\mathcal{C}\left[\begin{array}{cc}a_{+}+a_{-}-1 & A \\ -1 & B\end{array}\right]$ in the last line stem from Eisenstein-regularized sums which cancel from certain linear combination of terms with the same value of $a_{+}+a_{-}$, e.g.

$$
\begin{aligned}
& \mathcal{C}\left[\begin{array}{lll}
2 & 2 & A \\
0 & 0 & B
\end{array}\right]-2 \mathcal{C}\left[\begin{array}{lll}
3 & 1 & A \\
0 & 0 & B
\end{array}\right]=2 \mathcal{C}\left[\begin{array}{ll}
4 & A \\
0 & B
\end{array}\right]+3 \mathrm{G}_{4} \mathcal{C}\left[\begin{array}{l}
A \\
B
\end{array}\right] \\
& \mathcal{C}\left[\begin{array}{lll}
3 & 2 & A \\
0 & 0 & B
\end{array}\right]-3 \mathcal{C}\left[\begin{array}{lll}
4 & 1 & A \\
0 & 0 & B
\end{array}\right]=5 \mathcal{C}\left[\begin{array}{ll}
5 & A \\
0 & B
\end{array}\right]-3 \mathrm{G}_{4} \mathcal{C}\left[\begin{array}{ll}
1 & A \\
0 & B
\end{array}\right] \text {. }
\end{aligned}
$$




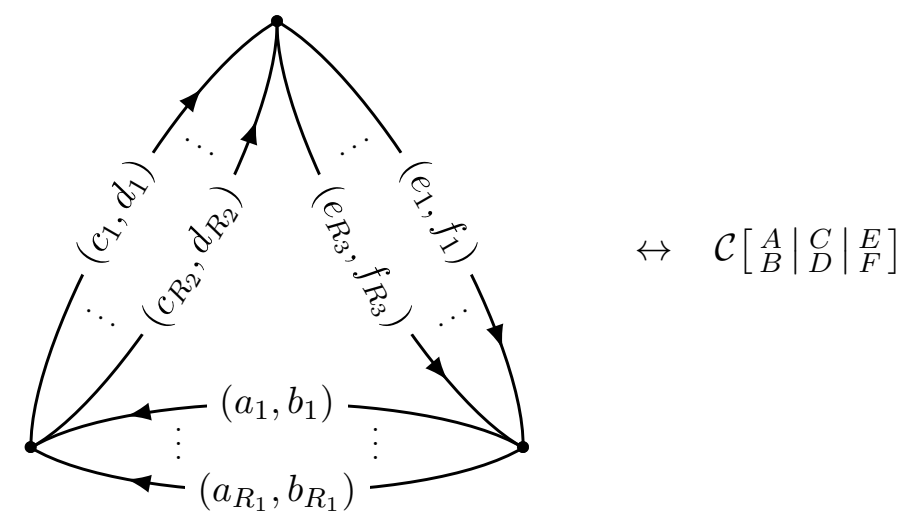

Figure 6. Graph associated to a trihedral modular graph form.

In fact, some of the integrals in the heterotic-string amplitude will involve the Eisensteinregularized contributions, namely

$$
\begin{aligned}
& \mathcal{C}\left[\begin{array}{lll}
2 & 1 & 2 \\
0 & 0 & 1
\end{array}\right]=3 \mathcal{C}\left[\begin{array}{ll}
5 & 0 \\
1 & 0
\end{array}\right]-\hat{\mathrm{G}}_{2} \mathcal{C}\left[\begin{array}{ll}
3 & 0 \\
1 & 0
\end{array}\right]-\mathrm{G}_{4} \\
& \mathcal{C}\left[\begin{array}{lll}
2 & 2 & 1 \\
0 & 0 & 1
\end{array}\right]=-6 \mathcal{C}\left[\begin{array}{ll}
5 & 0 \\
1 & 0
\end{array}\right]+2 \hat{\mathrm{G}}_{2} \mathcal{C}\left[\begin{array}{ll}
3 & 0 \\
1 & 0
\end{array}\right]+2 \mathrm{G}_{4} \\
& \mathcal{C}\left[\begin{array}{lll}
3 & 1 & 1 \\
0 & 0 & 1
\end{array}\right]=-4 \mathcal{C}\left[\begin{array}{ll}
5 & 0 \\
1 & 0
\end{array}\right]+\hat{\mathrm{G}}_{2} \mathcal{C}\left[\begin{array}{ll}
3 & 0 \\
1 & 0
\end{array}\right]+\mathrm{G}_{4}
\end{aligned}
$$

and

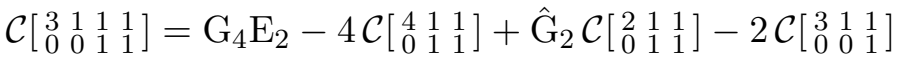

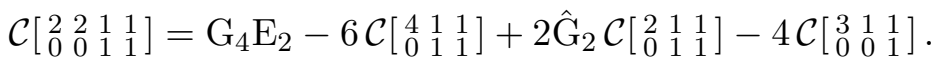

The right-hand sides of the identities (3.25) can be further simplified using the relations (3.21) and (A.1) as well as the holomorphic subgraph reductions (3.24).

\subsubsection{Trihedral modular graph forms}

We will also need modular graph forms descending from the trihedral topology. As depicted in figure 6, trihedral graphs are characterized by three vertices and three sets of edges each of which connects a different pair of vertices. We will denote the decorations for the $R_{1}, R_{2}$ and $R_{3}$ edges in the first, second and third set by $\left(a_{i}, b_{i}\right),\left(c_{i}, d_{i}\right)$ and $\left(e_{i}, f_{i}\right)$, respectively. By gathering the decorations $a_{1}, a_{2}, \ldots, a_{R_{1}}$ in a collective label $A$ (and a similar convention for $B, C, \ldots, F)$, the most general trihedral modular graph form can be written as

$$
\mathcal{C}\left[\begin{array}{c}
A \\
B
\end{array}\left|\begin{array}{l}
C \\
D
\end{array}\right| \underset{F}{E}\right]:=\left(\frac{\operatorname{Im} \tau}{\pi}\right)^{|B|+|D|+|F|} \sum_{\substack{p_{1}, p_{2}, \ldots, p_{R_{1}} \neq 0 \\
k_{1}, k_{2}, \ldots, k_{R_{2}} \neq 0 \\
\ell_{1}, \ell_{2}, \ldots, \ell_{R_{3}} \neq 0}} \frac{\delta\left(\sum_{i=1}^{R_{1}} p_{i}-\sum_{i=1}^{R_{2}} k_{i}\right) \delta\left(\sum_{i=1}^{R_{2}} k_{i}-\sum_{i=1}^{R_{3}} \ell_{i}\right)}{\left(\prod_{j=1}^{R_{1}} p_{j}^{a_{j}} \bar{p}_{j}^{b_{j}}\right)\left(\prod_{j=1}^{R_{2}} k_{j}^{c_{j}} \bar{k}_{j}^{d_{j}}\right)\left(\prod_{j=1}^{R_{3}} \ell_{j}^{e_{j}} \bar{\ell}_{j}^{f_{j}}\right)},
$$

where $|B|:=\sum_{i=1}^{R_{1}} b_{i}$ and similarly for $|D|$ and $|F|$, and the delta functions in the numerator enforce that each of the three sets of edges carries the same overall momentum, 
$\sum_{i=1}^{R_{1}} p_{i}=\sum_{i=1}^{R_{2}} k_{i}=\sum_{i=1}^{R_{3}} \ell_{i}$. Each vertex is taken to connect with more than two edges since the graph would otherwise be dihedral rather than trihedral. Accordingly, the simplest trihedral modular graph function contributing to (2.45) requires integration over five Green functions,

$$
D_{2,2,1}:=\int \mathrm{d} \mu_{4} G_{12}^{2} G_{23}^{2} G_{31}=\mathcal{C}\left[\begin{array}{llllll}
1 & 1 & 1 & 1 & 1 & 1 \\
1 & 1 & 1 & 1 & 1
\end{array}\right],
$$

whereas in the remaining integrals $\mathcal{I}_{\ldots}^{(w, 0)}$ in $(2.42)$, trihedral graphs arise from fewer Green functions. The ideas of momentum conservation and holomorphic subgraph reduction can be applied to generate identities between trihedral modular graph functions and more general graph topologies [66]. Examples and further details can be found in appendix A.4.

\subsubsection{Modular properties}

Under $\mathrm{SL}_{2}(\mathbb{Z})$ transformations, each factor of $p^{-a}$ and $\bar{p}^{-b}$ in the summand of modular graph forms contributes modular weight $(a, 0)$ and $(0, b)$, respectively, and $\operatorname{Im} \tau$ contributes modular weight $(-1,-1)$, i.e. for dihedral graphs

$$
\mathcal{C}\left[{ }_{B}^{A}\right]\left(\frac{\alpha \tau+\beta}{\gamma \tau+\delta}\right)=(\gamma \tau+\delta)^{|A|-|B|} \mathcal{C}\left[{ }_{B}^{A}\right](\tau)
$$

is a non-holomorphic modular form of weight $(|A|-|B|, 0)$. Here, we again use the notation $|A|=\sum_{i=1}^{R} a_{i}$ of (3.26). In the trihedral case, the analogous transformation is,

$$
\mathcal{C}\left[{ }_{B}^{A}\left|{ }_{D}^{C}\right|{ }_{F}^{E}\right]\left(\frac{\alpha \tau+\beta}{\gamma \tau+\delta}\right)=(\gamma \tau+\delta)^{|A|+|C|+|E|-|B|-|D|-|F|} \mathcal{C}\left[{ }_{B}^{A}\left|{ }_{D}^{C}\right|{ }_{F}^{E}\right](\tau) .
$$

\subsection{Differential equations and iterated Eisenstein integrals}

A major motivation for the introduction of modular graph forms in [15] is their descent from modular graph functions under Cauchy-Riemann derivatives

$$
\nabla:=2 i(\operatorname{Im} \tau)^{2} \frac{\partial}{\partial \tau}, \quad \bar{\nabla}:=-2 i(\operatorname{Im} \tau)^{2} \frac{\partial}{\partial \bar{\tau}} .
$$

These operators shift the weights of a non-holomorphic modular form ${ }^{12}$ according to $\nabla$ : $(0, w) \rightarrow(0, w-2)$ and $\bar{\nabla}:(w, 0) \rightarrow(w-2,0)$. By repeated action of $\nabla$ on modular graph functions, their relations including (3.7) can be elegantly proven based on properties of holomorphic modular forms up to integration constants [15]. Moreover, Cauchy-Riemann equations of this type are instrumental to generate the expansions of various modular graph forms around the cusp $\tau \rightarrow i \infty[22]$.

\subsubsection{Cauchy-Riemann derivatives of modular graph forms}

Cauchy-Riemann derivatives of the above lattice sums can be computed from

$$
\nabla\left(\frac{\operatorname{Im} \tau}{p}\right)=(\operatorname{Im} \tau)^{2} \frac{\bar{p}}{p^{2}}, \quad \bar{\nabla}\left(\frac{\operatorname{Im} \tau}{\bar{p}}\right)=(\operatorname{Im} \tau)^{2} \frac{p}{\bar{p}^{2}},
$$

\footnotetext{
${ }^{12}$ One can extend $\nabla$ to a map between modular forms of weights $\left(w_{1}, w_{2}\right) \rightarrow\left(w_{1}, w_{2}-2\right)$ by adding weight-dependent connection terms.
} 
which for instance relate non-holomorphic Eisenstein series to

$$
\nabla^{k} \mathrm{E}_{a}=\frac{(a+k-1) !}{(a-1) !} \frac{(\operatorname{Im} \tau)^{a+k}}{\pi^{a}} \sum_{p \neq 0} \frac{1}{p^{a+k} \bar{p}^{a-k}}=\frac{(a+k-1) !}{(a-1) !} \frac{(\operatorname{Im} \tau)^{2 k}}{\pi^{k}} \mathcal{C}\left[\begin{array}{cc}
a+k & 0 \\
a-k & 0
\end{array}\right]
$$

In particular, setting $k=a$ reproduces holomorphic Eisenstein series by (3.14),

$$
(\pi \nabla)^{k} \mathrm{E}_{k}=\frac{(2 k-1) !}{(k-1) !}(\operatorname{Im} \tau)^{2 k} \mathrm{G}_{2 k} .
$$

The simplest examples of these identities include

$$
\pi \nabla \mathrm{E}_{2}=2(\operatorname{Im} \tau)^{2} \mathcal{C}\left[\begin{array}{ll}
3 & 0 \\
1 & 0
\end{array}\right], \quad \pi \nabla \mathrm{E}_{3}=3(\operatorname{Im} \tau)^{2} \mathcal{C}\left[\begin{array}{ll}
4 & 0 \\
2 & 0
\end{array}\right], \quad(\pi \nabla)^{2} \mathrm{E}_{3}=12(\operatorname{Im} \tau)^{4} \mathcal{C}\left[\begin{array}{ll}
5 & 0 \\
1 & 0
\end{array}\right]
$$

as well as

$$
(\pi \nabla)^{2} \mathrm{E}_{2}=6(\operatorname{Im} \tau)^{4} \mathrm{G}_{4}, \quad(\pi \nabla)^{3} \mathrm{E}_{3}=60(\operatorname{Im} \tau)^{6} \mathrm{G}_{6} .
$$

For more general modular graph functions, iterated Cauchy-Riemann derivatives introduce combinations of holomorphic Eisenstein series and modular graph forms of lower complexity. For instance, the simplest irreducible two-loop graph function (3.5) obeys

$$
\mathrm{E}_{2,2}:=C_{2,1,1}-\frac{9}{10} \mathrm{E}_{4}, \quad(\pi \nabla)^{3} \mathrm{E}_{2,2}=-6(\operatorname{Im} \tau)^{4} \mathrm{G}_{4} \pi \nabla \mathrm{E}_{2},
$$

where $\mathrm{G}_{4}$ on the right-hand side stems from holomorphic subgraph reduction [15], and the subtraction of $\mathrm{E}_{4}$ has been tailored to simplify the differential equation [22].

More generally, the Cauchy-Riemann derivatives of dihedral modular graph forms as defined in (3.10) are given by [15]

$$
\begin{aligned}
\pi \nabla\left((\operatorname{Im} \tau)^{|A|-|B|} \mathcal{C}\left[\begin{array}{l}
A \\
B
\end{array}\right]\right) & =(\operatorname{Im} \tau)^{|A|-|B|+2} \sum_{j=1}^{R} a_{j} \mathcal{C}\left[\begin{array}{cccccc}
a_{1} & a_{2} & \ldots & a_{j}+1 & \ldots & a_{R} \\
b_{1} & b_{2} & \ldots & b_{j}-1 & \ldots & b_{R}
\end{array}\right] \\
\bar{\nabla} \mathcal{C}\left[\begin{array}{l}
A \\
B
\end{array}\right] & \left.=\pi \sum_{j=1}^{R} b_{j} \mathcal{C} C \begin{array}{llllll}
a_{1} & a_{2} & \ldots & a_{j}-1 & \ldots & a_{R} \\
b_{1} & b_{2} & \ldots & b_{j}+1 & \ldots & b_{R}
\end{array}\right],
\end{aligned}
$$

where the appearance of $\pi$ and $\operatorname{Im} \tau$ is due to our normalization convention (3.10) with holomorphic and antiholomorphic momenta entering on asymmetric footing. Negative entries $b_{j}-1<0$ on the right-hand side of (3.37) can be avoided using momentum conservation (3.18) if the left-hand side has already been simplified using holomorphic subgraph reduction (3.22) [15]. Similar differential equations for trihedral and more general modular graph forms are a straightforward consequence of (3.31).

\subsubsection{Iterated Eisenstein integrals}

Given that repeated $\tau$-derivatives of modular graph forms introduce factors of $\mathrm{G}_{k}$, it is natural to attempt to express modular graph forms in terms of iterated Eisenstein integrals 
with $k_{1}, k_{2}, \ldots, k_{r} \in 2 \mathbb{N}_{0}$,

$$
\begin{aligned}
\mathcal{E}_{0}\left(k_{1}, k_{2}, \ldots, k_{r} ; \tau\right) & :=2 \pi i \int_{\tau}^{i \infty} \mathrm{d} \tau_{r} \frac{\mathrm{G}_{k_{r}}^{0}\left(\tau_{r}\right)}{(2 \pi i)^{k_{r}}} \mathcal{E}_{0}\left(k_{1}, k_{2}, \ldots, k_{r-1} ; \tau_{r}\right) \\
& =(-1)^{r} \int_{0 \leq q_{1} \leq q_{2} \leq \ldots \leq q_{r} \leq q} \frac{\mathrm{d} q_{1}}{q_{1}} \frac{\mathrm{d} q_{2}}{q_{2}} \ldots \frac{\mathrm{d} q_{r}}{q_{r}} \frac{\mathrm{G}_{k_{1}}^{0}\left(\tau_{1}\right)}{(2 \pi i)^{k_{1}}} \frac{\mathrm{G}_{k_{2}}^{0}\left(\tau_{2}\right)}{(2 \pi i)^{k_{2}}} \ldots \frac{\mathrm{G}_{k_{r}}^{0}\left(\tau_{r}\right)}{(2 \pi i)^{k_{r}}} .
\end{aligned}
$$

The recursive definition in the first line is based on $\mathcal{E}_{0}(; \tau):=1$, and we have subtracted the zero modes in the $q$-expansion of the Eisenstein series $\mathrm{G}_{k}$ with even weight $k \neq 0$,

$$
\mathrm{G}_{k}^{0}:=\mathrm{G}_{k}-2 \zeta_{k}=\frac{2(2 \pi i)^{k}}{(k-1) !} \sum_{m, n=1}^{\infty} m^{k-1} q^{m n}, k \neq 0, \quad \mathrm{G}_{0}^{0}:=-1 .
$$

In this way, iterated Eisenstein integrals (3.38) are convergent if $k_{1} \neq 0$, and their expansion around the cusp can be inferred from straightforward integration [67]

$$
\begin{aligned}
& \mathcal{E}_{0}\left(k_{1}, 0^{p_{1}-1}, k_{2}, 0^{p_{2}-1}, \ldots, k_{r}, 0^{p_{r}-1} ; \tau\right)=(-2)^{r}\left(\prod_{j=1}^{r} \frac{1}{\left(k_{j}-1\right) !}\right) \\
& \quad \times \sum_{m_{i}, n_{i}=1}^{\infty} \frac{m_{1}^{k_{1}-1} m_{2}^{k_{2}-1} \ldots m_{r}^{k_{r}-1} q^{m_{1} n_{1}+m_{2} n_{2}+\ldots+m_{r} n_{r}}}{\left(m_{1} n_{1}\right)^{p_{1}}\left(m_{1} n_{1}+m_{2} n_{2}\right)^{p_{2}} \ldots\left(m_{1} n_{1}+m_{2} n_{2}+\ldots+m_{r} n_{r}\right)^{p_{r}}},
\end{aligned}
$$

where $k_{j} \neq 0 \forall j=1,2, \ldots, r$, and $0^{p}$ is a shorthand for $p$ successive zeros. The number $r$ of non-zero entries on the left-hand side of (3.40) is also referred to as the depth of an iterated Eisenstein integral as in (3.38). With these definitions, (3.33) can be integrated ${ }^{13}$ to the following representations of non-holomorphic Eisenstein series $[8,22,68]$

$$
\begin{aligned}
\mathrm{E}_{2}= & \frac{y^{2}}{45}+\frac{\zeta_{3}}{y}-12 \operatorname{Re}\left[\mathcal{E}_{0}(4,0)\right]-\frac{6}{y} \operatorname{Re}\left[\mathcal{E}_{0}(4,0,0)\right] \\
\mathrm{E}_{3}= & \frac{2 y^{3}}{945}+\frac{3 \zeta_{5}}{4 y^{2}}-120 \operatorname{Re}\left[\mathcal{E}_{0}(6,0,0)\right]-\frac{180}{y} \operatorname{Re}\left[\mathcal{E}_{0}(6,0,0,0)\right]-\frac{90}{y^{2}} \operatorname{Re}\left[\mathcal{E}_{0}(6,0,0,0,0)\right] \\
\mathrm{E}_{4}= & \frac{y^{4}}{4725}+\frac{5 \zeta_{7}}{8 y^{3}}-1680 \operatorname{Re}\left[\mathcal{E}_{0}(8,0,0,0)\right]-\frac{5040}{y} \operatorname{Re}\left[\mathcal{E}_{0}(8,0,0,0,0)\right] \\
& \quad-\frac{6300}{y^{2}} \operatorname{Re}\left[\mathcal{E}_{0}(8,0,0,0,0,0)\right]-\frac{3150}{y^{3}} \operatorname{Re}\left[\mathcal{E}_{0}(8,0,0,0,0,0,0)\right]
\end{aligned}
$$

Here and in later equations, we are using the shorthand

$$
y:=\pi \operatorname{Im} \tau,
$$

and the Riemann zeta values in (3.41) arise as integration constants whose coefficients can be determined from modular invariance. The modular transformations of the iterated Eisenstein integrals (3.38) has been discussed in [22, 69].

\footnotetext{
${ }^{13}$ In performing these integrations, it is useful to note that [22]

$$
\pi \nabla\left(y^{n}\right)=n y^{n+1}, \quad \pi \nabla\left(\mathcal{E}_{0}\left(k_{1}, k_{2}, \ldots, k_{r}\right)\right)=\frac{4 y^{2} \mathrm{G}_{k_{r}}^{0}}{(2 \pi i)^{k_{r}}} \mathcal{E}_{0}\left(k_{1}, k_{2}, \ldots, k_{r}\right) .
$$
}


For instance, integration of (3.36) yields the following representation of the simplest irreducible two-loop graph function [22]

$$
\begin{aligned}
\mathrm{E}_{2,2}= & -\frac{y^{4}}{20250}+\frac{y \zeta_{3}}{45}+\frac{5 \zeta_{5}}{12 y}-\frac{\zeta_{3}^{2}}{4 y^{2}}-\left(\frac{2 y}{15}-\frac{3 \zeta_{3}}{y^{2}}\right) \operatorname{Re}\left[\mathcal{E}_{0}(4,0,0)\right] \\
& -\frac{9 \operatorname{Re}\left[\mathcal{E}_{0}(4,0,0)\right]^{2}}{y^{2}}-72 \operatorname{Re}\left[\mathcal{E}_{0}(4,4,0,0)\right]-\frac{1}{5} \operatorname{Re}\left[\mathcal{E}_{0}(4,0,0,0)\right] \\
& -\frac{36 \operatorname{Re}\left[\mathcal{E}_{0}(4,0,4,0,0)\right]}{y}-\frac{108 \operatorname{Re}\left[\mathcal{E}_{0}(4,4,0,0,0)\right]}{y}-\frac{\operatorname{Re}\left[\mathcal{E}_{0}(4,0,0,0,0)\right]}{10 y},
\end{aligned}
$$

where the coefficients of the zeta values are again fixed by modular invariance. The corresponding $\mathcal{E}_{0}$-representations of the first Cauchy-Riemann derivatives $\pi \nabla \mathrm{E}_{\ldots}$ are spelled out in (5.31) below.

All the modular graph forms $\mathcal{C}[\Gamma]$ that will be encountered in the $\alpha^{\prime}$-expansions of section 4 have been reduced to iterated Eisenstein integrals with Laurent polynomials in $y$ as their coefficients. In these cases, the $q$-series representations (3.40) of iterated Eisenstein integrals give rise to an expansion

$$
\mathcal{C}[\Gamma]=\sum_{m, n=0}^{\infty} c_{m, n}^{\Gamma}(y) q^{m} \bar{q}^{n}
$$

where $\Gamma$ may represent the labels of the dihedral and trihedral cases (3.10) and (3.26) or a more general graph. The coefficients $c_{m, n}^{\Gamma}(y)$ are Laurent polynomials in $y$, and the terms in (3.41) and (3.43) without any factor of $\operatorname{Re} \mathcal{E}_{0}$ reproduce the zero modes $c_{0,0}^{\Gamma}(y)$ of the simplest modular graph functions [10].

From the methods of [15], an expansion of the form (3.44) is expected to exist for any modular graph form, and a proof has been given in $[14,70]$ for the special case of modular graph functions. Indeed, the proof of the references can be adapted to general modular graph forms ${ }^{14}$ including global bounds (which only depend on the graph $\Gamma$ but not on $m$ and $n$ ) on the highest and lowest powers of $y$ in the above $c_{m, n}^{\Gamma}(y)$. Note that, for modular graph functions, the Laurent coefficients within $c_{m, n}^{\Gamma}(y)$ are proven to be $\mathbb{Q}$-linear combinations of cyclotomic MZVs and conjectured to be single-valued MZVs [14, 70].

A major motivation for expressing modular graph forms in terms of iterated Eisenstein integrals is the connection with open-string amplitudes. As will be detailed in section 5 , the $\alpha^{\prime}$-expansion of the iterated integrals over open-string punctures yields elliptic multiple zeta values $[6,7]$ which are in turn expressible in terms of the $\mathcal{E}_{0}$ in $(3.38)[5,67]$. The representations (3.41) and (3.43) of modular graph functions have been connected with open-string quantities through a candidate prescription for an elliptic single-valued map [22]. In section 5 , we will find a similar correspondence between open strings and the integral $\mathcal{I}_{1234}^{(2,0)}$ in (2.44) which enters the $\tau$-integrand (2.42) of the heterotic-string amplitude.

\footnotetext{
${ }^{14}$ We are grateful to Federico Zerbini for discussions on this point.
} 


\section{$4 \alpha^{\prime}$-expansion of the heterotic-string amplitude and modular graph forms}

This section is dedicated to the low-energy expansion of the four-point integrals $\mathcal{I}_{\text {... }}^{(w)}$ defined in (2.43) to (2.47) which arise in the gauge sector of the heterotic string. We will give general arguments in section 4.1 that each order in their $\alpha^{\prime}$-expansion is expressible via modular graph forms. The low-energy expansion of the modular invariant integral $\mathcal{I}^{(0,0)}$ is well-studied in the literature, see (3.9) for the leading orders. The integrals $\mathcal{I}_{1234}^{(w, 0)}$ and $\mathcal{I}_{12 \mid 34}^{(w, 0)}$ from the planar and the non-planar sector of (2.42) are expanded in sections 4.2 and 4.3, respectively. We will elaborate on their transcendentality properties in section 4.4 and perform the integrals over $\tau$ in the amplitude (2.38) up to the order of $\alpha^{\prime 2}$ in section 4.5. Finally, it will be explained in section 4.6 why the integrals over the punctures in all massless $n$-point amplitudes boil down to modular graph forms.

\section{1 $\alpha^{\prime}$-expansions from modular graph forms}

Our constructive method to extract modular graph forms from the $\alpha^{\prime}$-expansions of (2.43) to (2.47) starts from the double Fourier expansion of the non-holomorphic KroneckerEisenstein series (2.4),

$$
\Omega(z, \beta, \tau)=\sum_{m, n \in \mathbb{Z}} \frac{e^{2 \pi i(m v-n u)}}{m \tau+n+\beta},
$$

where again $z=u \tau+v$. After removing the contribution $\beta^{-1}$ due the origin $(m, n)=(0,0)$, a geometric-series expansion in $\beta$ implies the lattice-sum representation of the doublyperiodic functions in (2.5),

$$
f^{(w)}(z, \tau)=(-1)^{w-1} \sum_{(m, n) \neq(0,0)} \frac{e^{2 \pi i(m v-n u)}}{(m \tau+n)^{w}}, \quad w \geq 1,
$$

which exposes their modular properties (2.9). The lattice sums at $w=1,2$ are not absolutely convergent on the entire torus, and we will comment on regularization prescriptions in section 4.3. Still, the sum at $w=1$ is formally consistent with (2.16) by taking the holomorphic derivative

$$
-\frac{\operatorname{Im} \tau}{\pi} \partial_{z} \frac{e^{2 \pi i(m v-n u)}}{|m \tau+n|^{2}}=\frac{e^{2 \pi i(m v-n u)}}{m \tau+n}
$$

of the summand of the Green function (3.1). The single pole of $f^{(1)}(z, \tau)$ at $z=0$ mentioned below (2.5) is not obvious in the lattice-sum representation (4.2) since the limit $z \rightarrow 0$ does not commute with the sum.

The lattice-sum representation (4.2) manifests that the holomorphic Eisenstein series $\mathrm{G}_{k}$ in (2.31) are recovered when evaluating the even-weight $f^{(w)}$ at the origin,

$$
f^{(k)}(0, \tau)=-\sum_{(m, n) \neq(0,0)} \frac{1}{(m \tau+n)^{k}}=-\mathrm{G}_{k}(\tau), \quad k \geq 4 .
$$

At $w=2$, however, the limit $u, v \rightarrow 0$ introduces conditionally convergent sums, and one can obtain a holomorphic but non-modular expression (3.15). 


\subsubsection{Fourier expansion of the $V_{w}$ functions}

The elliptic integrands $V_{w}$ of $\mathcal{I}^{(w, 0)}$ are defined by cyclic products of Kronecker-Eisenstein series, see (2.6). Hence, the dependence of $V_{w}$ on the punctures can be brought into the same plane-wave form (4.1) as the Green function (3.1). In the planar cases $\mathcal{I}_{1234}^{(w, 0)}$ with $w=2,4$, for instance, the Fourier expansion (4.1) propagates to

$$
\begin{aligned}
& V_{w}(1,2,3,4)=\left.\sum_{\substack{m_{1}, m_{2}, m_{3}, m_{4} \in \mathbb{Z} \\
n_{1}, n_{2}, n_{3}, n_{4} \in \mathbb{Z}}} \frac{1}{\left(m_{1} \tau+n_{1}+\beta\right)\left(m_{2} \tau+n_{2}+\beta\right)\left(m_{3} \tau+n_{3}+\beta\right)\left(m_{4} \tau+n_{4}+\beta\right)}\right|_{\beta^{w-4}} \\
& \times e^{2 \pi i\left[\left(m_{1}-m_{4}\right) v_{12}+\left(m_{2}-m_{4}\right) v_{23}+\left(m_{3}-m_{4}\right) v_{34}-\left(n_{1}-n_{4}\right) u_{12}-\left(n_{2}-n_{4}\right) u_{23}-\left(n_{3}-n_{4}\right) u_{34}\right]}
\end{aligned}
$$

with $z_{j}=u_{j} \tau+v_{j}$ as well as $u_{i j}=u_{i}-u_{j}$ and $v_{i j}=v_{i}-v_{j}$. With the following notation for the scalar product involving discrete momenta $p_{i}=m_{i} \tau+n_{i}$,

$$
\left\langle p_{i}, z_{j}\right\rangle:=m_{i} v_{j}-n_{i} u_{j}
$$

the representation (4.5) of the $V_{w}$ functions shares the lattice sums of modular graph forms, see section 3.2 ,

$$
V_{w}(1,2,3,4)=\left.\sum_{p_{1}, p_{2}, p_{3}, p_{4}} \frac{e^{2 \pi i\left(\left\langle p_{1}-p_{4}, z_{12}\right\rangle+\left\langle p_{2}-p_{4}, z_{23}\right\rangle+\left\langle p_{3}-p_{4}, z_{34}\right\rangle\right)}}{\left(p_{1}+\beta\right)\left(p_{2}+\beta\right)\left(p_{3}+\beta\right)\left(p_{4}+\beta\right)}\right|_{\beta^{w-4}} .
$$

Laurent expansion in $\beta$ will generate arbitrary powers of inverse holomorphic momenta,

$$
\sum_{p} \frac{1}{p+\beta}=\frac{1}{\beta}+\sum_{p \neq 0} \frac{1}{p+\beta}=\frac{1}{\beta}+\sum_{p \neq 0}\left(\frac{1}{p}-\frac{\beta}{p^{2}}+\frac{\beta^{2}}{p^{3}}-\frac{\beta^{3}}{p^{4}}+\ldots\right)=\frac{1}{\beta}+\sum_{p \neq 0} \sum_{a=1}^{\infty} \frac{(-\beta)^{a-1}}{p^{a}},
$$

which correspond to decorated edges $(a, 0)$ in the discussion of modular graph forms. However, in contrast to the lattice-sum definition of modular graph forms, the sums over $p_{j}$ in (4.7) include the origin and must be decomposed according to $\sum_{p_{j}} f\left(p_{j}\right)=$ $f(0)+\sum_{p_{j} \neq 0} f\left(p_{j}\right)$ before making contact with modular graph forms.

The integrands in the non-planar sector of $(2.42)$ boil down to $V_{2}(i, j)$ with the even simpler Fourier expansion

$$
\begin{aligned}
V_{2}(i, j) & =-\wp\left(z_{i j}, \tau\right)=\hat{\mathrm{G}}_{2}-\partial_{z_{i j}}^{2} G\left(z_{i j}, \tau\right) \\
& =\hat{\mathrm{G}}_{2}-\frac{\pi}{\operatorname{Im} \tau} \sum_{(m, n) \neq(0,0)} \frac{m \bar{\tau}+n}{m \tau+n} e^{2 \pi i\left(m v_{i j}-n u_{i j}\right)} \\
& =\hat{\mathrm{G}}_{2}-\frac{\pi}{\operatorname{Im} \tau} \sum_{p \neq 0} \frac{\bar{p}}{p} e^{2 \pi i\left\langle p, z_{i j}\right\rangle}
\end{aligned}
$$

\subsubsection{Heterotic graph forms}

By (4.9) and (4.7), each term in the Taylor expansion of the integrals $\mathcal{I}_{\ldots}^{(w, 0)}$ in $(2.43)$ to (2.47) boils down to straightforward Fourier integrals over $z_{j}=u_{j} \tau+v_{j}$,

$$
\int \mathrm{d} \mu_{4}=\prod_{j=1}^{3} \int_{0}^{1} \mathrm{~d} u_{j, j+1} \int_{0}^{1} \mathrm{~d} v_{j, j+1} \quad \Longrightarrow \quad \int \mathrm{d} \mu_{4} e^{2 \pi i\left(\left\langle p, z_{12}\right\rangle+\left\langle k, z_{23}\right\rangle+\left\langle\ell, z_{34}\right\rangle\right)}=\delta(p) \delta(k) \delta(\ell)
$$


which collapse some of the above sums over $p_{i}$ through the delta functions on the right-hand side. Hence, integrals of this type over (4.9) and each term in the Laurent expansion of (4.7) w.r.t. $\beta$ line up with the framework of modular graph forms: different types of decorated edges arise from the Green functions $G_{i j}$ of the Koba-Nielsen factor, the Laurent-expanded $\frac{1}{p_{j}+\beta}$ in (4.7) and the ratio $\frac{\bar{p}}{p}$ in (4.9). Moreover, the integrations (4.10) yield momentum conserving vertices.

In order to track the different contributions from the expanded Koba-Nielsen factor to $\mathcal{I}_{1234}^{(w, 0)}$, we introduce the following function of $\beta$ for each monomial in Green functions $G_{i j}$,

$$
\begin{aligned}
H_{1234}\left[\prod_{i<j} G_{i j}^{n_{i j}} ; \beta\right] & =\int \mathrm{d} \mu_{4} \Omega\left(z_{12}, \beta, \tau\right) \Omega\left(z_{23}, \beta, \tau\right) \Omega\left(z_{34}, \beta, \tau\right) \Omega\left(z_{41}, \beta, \tau\right) \prod_{i<j} G_{i j}^{n_{i j}} \\
& =\sum_{p_{1}, p_{2}, p_{3}, p_{4}} \int \mathrm{d} \mu_{4} \frac{e^{2 \pi i\left(\left\langle p_{1}-p_{4}, z_{12}\right\rangle+\left\langle p_{2}-p_{4}, z_{23}\right\rangle+\left\langle p_{3}-p_{4}, z_{34}\right\rangle\right)}}{\left(p_{1}+\beta\right)\left(p_{2}+\beta\right)\left(p_{3}+\beta\right)\left(p_{4}+\beta\right)} \prod_{i<j} G_{i j}^{n_{i j}} \\
& =H_{1234}^{(0,0)}\left[\prod_{i<j} G_{i j}^{n_{i j}}\right] \frac{1}{\beta^{4}}+H_{1234}^{(2,0)}\left[\prod_{i<j} G_{i j}^{n_{i j}}\right] \frac{1}{\beta^{2}}+H_{1234}^{(4,0)}\left[\prod_{i<j} G_{i j}^{n_{i j}}\right]+\mathcal{O}\left(\beta^{2}\right) .
\end{aligned}
$$

Since $H_{1234}[\ldots ; \beta]$ is an even function of $\beta$, odd powers of $\beta$ are absent in the Laurent expansions in the third line. The latter define the contributions $H_{1234}^{(w, 0)}[\ldots]$ relevant to $\mathcal{I}_{1234}^{(w, 0)}\left(\right.$ with $w=0,2,4$ and $V_{0}(1,2,3,4)=1$ ),

$$
H_{1234}^{(w, 0)}\left[\prod_{i<j} G_{i j}^{n_{i j}}\right]=\int \mathrm{d} \mu_{4} V_{w}(1,2,3,4) \prod_{i<j} G_{i j}^{n_{i j}}
$$

With the above definitions, the leading orders of the $\alpha^{\prime}$-expansions of $\mathcal{I}_{1234}^{(w, 0)}$ read

$$
\mathcal{I}_{1234}^{(w, 0)}\left(s_{i j}, \tau\right)=H_{1234}^{(w, 0)}[\emptyset]+\sum_{1 \leq i<j}^{4} s_{i j} H_{1234}^{(w, 0)}\left[G_{i j}\right]+\frac{1}{2} \sum_{\substack{1 \leq i<j \\ 1 \leq k<l}}^{4} s_{i j} s_{k l} H_{1234}^{(w, 0)}\left[G_{i j} G_{k l}\right]+\mathcal{O}\left(\alpha^{\prime 3}\right)
$$

Analogous definitions can be made in the non-planar sector, where the bookkeeping variable $\beta$ can be bypassed from the simple form (4.9) of $V_{2}(i, j)$,

$$
\begin{aligned}
& H_{12 \mid 34}^{(4,0)}\left[\prod_{i<j} G_{i j}^{n_{i j}}\right]=\int \mathrm{d} \mu_{4} V_{2}(1,2) V_{2}(3,4) \prod_{i<j} G_{i j}^{n_{i j}} \\
& H_{12 \mid 34}^{(2,0)}\left[\prod_{i<j} G_{i j}^{n_{i j}}\right]=\int \mathrm{d} \mu_{4}\left[V_{2}(1,2)+V_{2}(3,4)\right] \prod_{i<j} G_{i j}^{n_{i j}},
\end{aligned}
$$

and (4.13) with $1234 \rightarrow 12 \mid 34$ applies to the $\alpha^{\prime}$-expansions of the non-planar integrals $\mathcal{I}_{12 \mid 34}^{(w, 0)}$.

By analogy with the definition of modular graph functions through the $\alpha^{\prime}$-expansion of $\mathcal{I}^{(0,0)}$ in $(2.45)$, we will refer to the above $H_{1234}^{(w, 0)}[\ldots]$ and $H_{12 \mid 34}^{(w, 0)}[\ldots]$ as heterotic graph forms. They are modular graph forms of weight $(w, 0)$, as one can see from modular invariance of $G_{i j}$ and $\mathrm{d} \mu_{4}$ and the weight $(w, 0)$ of $V_{w}$. Modular graph functions are recovered from the weight-zero instances $H_{1234}^{(0,0)}[\ldots]$. 


\begin{tabular}{|cc|}
\hline Order & Inequivalent planar heterotic graph forms \\
\hline$\alpha^{\prime 0}$ & $H_{1234}^{(w, 0)}[\emptyset]$ \\
$\alpha^{\prime 1}$ & $H_{1234}^{(w, 0)}\left[G_{12}\right], H_{1234}^{(w, 0)}\left[G_{13}\right]$ \\
$\alpha^{\prime 2}$ & $H_{1234}^{(w, 0)}\left[G_{12}^{2}\right], H_{1234}^{(w, 0)}\left[G_{13} G_{24}\right], H_{1234}^{(w, 0)}\left[G_{12} G_{13}\right]$ \\
& $H_{1234}^{(w, 0)}\left[G_{13}^{2}\right], H_{1234}^{(w, 0)}\left[G_{12} G_{34}\right], H_{1234}^{(w, 0)}\left[G_{12} G_{23}\right]$ \\
$\alpha^{\prime 3}$ & $H_{1234}^{(w, 0)}\left[G_{12}^{3}\right], H_{1234}^{(w, 0)}\left[G_{13}^{2} G_{24}\right], H_{1234}^{(w, 0)}\left[G_{12} G_{23} G_{34}\right], H_{1234}^{(w, 0)}\left[G_{12} G_{23} G_{31}\right]$ \\
& $H_{1234}^{(w, 0)}\left[G_{13}^{3}\right], H_{1234}^{(w, 0)}\left[G_{12}^{2} G_{34}\right], H_{1234}^{(w, 0)}\left[G_{12} G_{13} G_{14}\right], H_{1234}^{(w, 0)}\left[G_{12} G_{13} G_{34}\right]$ \\
& $H_{1234}^{(w, 0)}\left[G_{12}^{2} G_{13}\right], H_{1234}^{(w, 0)}\left[G_{12} G_{13}^{2}\right], H_{1234}^{(w, 0)}\left[G_{12}^{2} G_{23}\right], H_{1234}^{(w, 0)}\left[G_{12} G_{13} G_{24}\right]$ \\
\hline
\end{tabular}

Table 1. Inequivalent planar heterotic graph forms with respect to the dihedral symmetry.

Note that similar techniques have been applied in [24] ${ }^{15}$ to evaluate certain integrals over the punctures in five- and six-point gauge correlators at $\exp \left(\sum_{i<j} s_{i j} G_{i j}\right) \rightarrow 1$ that demonstrate the absence of $\operatorname{Tr}\left(F^{5}\right)$ and $\operatorname{Tr}\left(F^{6}\right)$ operators in the one-loop effective action.

\subsection{Planar heterotic graph forms}

In this section, we compute and simplify the planar heterotic graph forms that arise from the Koba-Nielsen integrals over $V_{w}(1,2,3,4)$ at the leading orders in $\alpha^{\prime}$ and $w=2,4$. These results follow from Laurent expansion of (4.11), but one can equivalently employ the representation of the $V_{w}$ functions in terms of the doubly-periodic $f^{(w)}$ with Fourier expansion in (4.2). In a shorthand with $f_{i j}^{(w)}:=f^{(w)}\left(z_{i}-z_{j}, \tau\right)$, the relevant integrands are

$$
\begin{aligned}
& V_{2}(1,2,3,4)=f_{12}^{(1)} f_{34}^{(1)}+f_{23}^{(1)} f_{41}^{(1)}+\left[f_{12}^{(2)}+f_{12}^{(1)} f_{23}^{(1)}+\operatorname{cyc}(1,2,3,4)\right] \\
& V_{4}(1,2,3,4)=f_{12}^{(1)} f_{23}^{(1)} f_{34}^{(1)} f_{41}^{(1)}+f_{12}^{(1)} f_{34}^{(3)}+f_{12}^{(2)} f_{34}^{(2)}+f_{12}^{(3)} f_{34}^{(1)}+f_{23}^{(1)} f_{41}^{(3)}+f_{23}^{(2)} f_{41}^{(2)}+f_{23}^{(3)} f_{41}^{(1)} \\
& +\left[f_{12}^{(1)} f_{23}^{(1)} f_{34}^{(2)}+f_{12}^{(1)} f_{23}^{(2)} f_{34}^{(1)}+f_{12}^{(2)} f_{23}^{(1)} f_{34}^{(1)}+f_{12}^{(1)} f_{23}^{(3)}+f_{12}^{(2)} f_{23}^{(2)}+f_{12}^{(3)} f_{23}^{(1)}+f_{12}^{(4)}+\operatorname{cyc}(1,2,3,4)\right] .
\end{aligned}
$$

By permutation symmetry of the Koba-Nielsen factor, the dihedral symmetry (2.7) of the $V_{w}$ functions propagates to the integrals $(w=2,4)$,

$$
\mathcal{I}_{1234}^{(w, 0)}=\mathcal{I}_{4321}^{(w, 0)}, \quad \mathcal{I}_{1234}^{(w, 0)}=\mathcal{I}_{2341}^{(w, 0)} .
$$

On these grounds, only a small fraction of heterotic graph forms at a given order in $\alpha^{\prime}$ are inequivalent under dihedral symmetry, see table 1 . In the remainder of this subsection, we will restrict our attention to the heterotic graph forms in the table.

\subsubsection{Leading orders $\alpha^{\prime 0}$ and $\alpha^{\prime 1}$}

In the absence of any $G_{i j}$ in the integrand, we obtain the simplest heterotic graph forms

$$
H_{1234}^{(2,0)}[\emptyset]=0, \quad H_{1234}^{(4,0)}[\emptyset]=\mathrm{G}_{4},
$$

\footnotetext{
${ }^{15}$ The integrations in [24] are performed before summing over the spin structure (2.24), based on a double Fourier expansion of the Szegö kernel similar to (4.1).
} 
where the Eisenstein series $\mathrm{G}_{4}$ on the right-hand side can be traced back to the contribution of $f_{12}^{(1)} f_{23}^{(1)} f_{34}^{(1)} f_{41}^{(1)}$ in (4.15), see [23]. At first order in $\alpha^{\prime}$, the two inequivalent heterotic graph forms in table 1 are found to be

$$
H_{1234}^{(2,0)}\left[G_{12}\right]=-\mathcal{C}\left[\begin{array}{ll}
3 & 0 \\
1 & 0
\end{array}\right], \quad H_{1234}^{(2,0)}\left[G_{13}\right]=2 \mathcal{C}\left[\begin{array}{ll}
3 & 0 \\
1 & 0
\end{array}\right],
$$

as well as

$$
H_{1234}^{(4,0)}\left[G_{12}\right]=-\mathcal{C}\left[\begin{array}{lll}
3 & 1 & 1 \\
0 & 0 & 1
\end{array}\right]-4 \mathcal{C}\left[\begin{array}{ll}
5 & 0 \\
1 & 0
\end{array}\right], \quad H_{1234}^{(4,0)}\left[G_{13}\right]=\mathcal{C}\left[\begin{array}{lll}
2 & 2 & 1 \\
0 & 0 & 1
\end{array}\right]+6 \mathcal{C}\left[\begin{array}{ll}
5 & 0 \\
1 & 0
\end{array}\right],
$$

see (3.10) for the dihedral modular graph forms $\mathcal{C}[\ldots]$. The modular graph forms associated with three-edge graphs can be simplified via holomorphic subgraph reduction (3.24), and the $H_{1234}^{(4,0)}\left[G_{i j}\right]$ can be expressed solely in terms of single lattice sums (just like the $\left.H_{1234}^{(2,0)}\left[G_{i j}\right]\right)$,

$$
H_{1234}^{(4,0)}\left[G_{12}\right]=-\hat{\mathrm{G}}_{2} \mathcal{C}\left[\begin{array}{ll}
3 & 0 \\
1 & 0
\end{array}\right]-\mathrm{G}_{4}, \quad H_{1234}^{(4,0)}\left[G_{13}\right]=2 \hat{\mathrm{G}}_{2} \mathcal{C}\left[\begin{array}{ll}
3 & 0 \\
1 & 0
\end{array}\right]+2 \mathrm{G}_{4}
$$

\subsubsection{Subleading orders $\alpha^{\prime 2}$ and $\alpha^{3}$}

At the order $\alpha^{\prime 2}$, the six inequivalent heterotic graph forms in table 1 evaluate to

$$
\begin{aligned}
& H_{1234}^{(2,0)}\left[G_{12}^{2}\right]=-\mathcal{C}\left[\begin{array}{lll}
2 & 1 & 1 \\
0 & 1 & 1
\end{array}\right] \\
& H_{1234}^{(2,0)}\left[G_{13}^{2}\right]=2 \mathcal{C}\left[\begin{array}{lll}
2 & 1 & 1 \\
0 & 1 & 1
\end{array}\right] \\
& H_{1234}^{(2,0)}\left[G_{13} G_{24}\right]=-2 \mathcal{C}\left[\begin{array}{ll}
4 & 0 \\
2 & 0
\end{array}\right] \\
& H_{1234}^{(2,0)}\left[G_{13} G_{12}\right]=-\mathcal{C}\left[\begin{array}{ll}
4 & 0 \\
2 & 0
\end{array}\right]-\mathcal{C}\left[\begin{array}{lll}
2 & 1 & 1 \\
1 & 1 & 0
\end{array}\right] \\
& H_{1234}^{(2,0)}\left[G_{12} G_{34}\right]=\mathcal{C}\left[\begin{array}{ll}
4 & 0 \\
2 & 0
\end{array}\right] \\
& H_{1234}^{(2,0)}\left[G_{12} G_{23}\right]=\mathcal{C}\left[\begin{array}{ll}
4 & 0 \\
2 & 0
\end{array}\right] \text {, }
\end{aligned}
$$

as well as

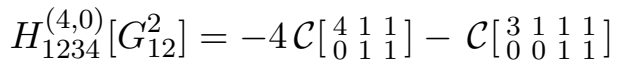

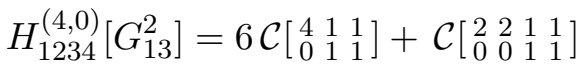

$$
\begin{aligned}
& H_{1234}^{(4,0)}\left[G_{13} G_{24}\right]=-2 \mathcal{C}\left[\begin{array}{cc}
6 & 0 \\
2 & 0
\end{array}\right]-4 \mathcal{C}\left[\begin{array}{lll}
2 & 2 & 2 \\
1 & 1 & 0
\end{array}\right]+8 \mathcal{C}\left[\begin{array}{lll}
3 & 2 & 1 \\
1 & 1 & 0
\end{array}\right]+\mathcal{C}_{\text {tet }} \\
& H_{1234}^{(4,0)}\left[G_{13} G_{12}\right]=-\mathcal{C}\left[\begin{array}{ll}
6 & 0 \\
2 & 0
\end{array}\right]+\mathcal{C}\left[\begin{array}{lll}
3 & 2 & 1 \\
0 & 1 & 1
\end{array}\right]-\mathcal{C}\left[\begin{array}{lll}
4 & 1 & 1 \\
1 & 1 & 0
\end{array}\right]+\mathcal{C}\left[\begin{array}{llll|l}
2 & 1 & 1 & 1 & 1 \\
0 & 1 & 1 & 0 & 0
\end{array}\right]-\left(\mathcal{C}\left[\begin{array}{ll}
3 & 0 \\
1 & 0
\end{array}\right]\right)^{2} \\
& H_{1234}^{(4,0)}\left[G_{12} G_{34}\right]=3 \mathcal{C}\left[\begin{array}{ll}
6 & 0 \\
2 & 0
\end{array}\right]+4 \mathcal{C}\left[\begin{array}{lll}
4 & 1 & 1 \\
1 & 1 & 0
\end{array}\right]-2 \mathcal{C}\left[\begin{array}{lll}
3 & 2 & 1 \\
1 & 0 & 1
\end{array}\right]+\left(\mathcal{C}\left[\begin{array}{ll}
3 & 0 \\
1 & 0
\end{array}\right]\right)^{2}+\mathcal{C}\left[\begin{array}{llll}
1 & 1 & 1 & 1 \\
1 & 0 & 1 & 0 \\
1 & 0
\end{array}\right]
\end{aligned}
$$

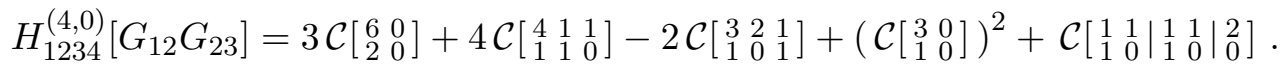

At this order, the contribution of $f_{12}^{(1)} f_{23}^{(1)} f_{34}^{(1)} f_{41}^{(1)}$ to $V_{4}(1,2,3,4)$ introduces trihedral modular graph forms (3.26) as well as the following tetrahedral representative in $H_{1234}^{(4,0)}\left[G_{13} G_{24}\right]$

$$
\mathcal{C}_{\text {tet }}:=\left(\frac{\operatorname{Im} \tau}{\pi}\right)_{\substack{p_{1}, p_{2}, p_{3} \neq 0 \\ p_{1}+p_{3} \neq 0 \\ p_{2}+p_{3} \neq 0 \\ p_{1}+p_{2}+p_{3} \neq 0}}^{2} \frac{1}{\left|p_{1}\right|^{2}\left|p_{2}\right|^{2} p_{3}\left(p_{1}+p_{3}\right)\left(p_{2}+p_{3}\right)\left(p_{1}+p_{2}+p_{3}\right)} .
$$


Similar to (4.20), the complexity of the lattice sums in $H_{1234}^{(w, 0)}\left[G_{i j} G_{k l}\right]$ can be considerably reduced: the three-edge sums at $w=2$ boil down to $\mathcal{C}\left[\begin{array}{ll}4 & 0 \\ 2 & 0\end{array}\right]$ via (3.21), e.g.

$$
H_{1234}^{(2,0)}\left[G_{12}^{2}\right]=-\mathcal{C}\left[\begin{array}{cc}
4 & 0 \\
2 & 0
\end{array}\right], \quad H_{1234}^{(2,0)}\left[G_{13} G_{12}\right]=-\frac{1}{2} \mathcal{C}\left[\begin{array}{cc}
4 & 0 \\
2 & 0
\end{array}\right]
$$

Using the techniques of the appendices A.4 and A.5, the trihedral and tetrahedral topologies at $w=4$ can be expressed in terms of single lattice sums as well,

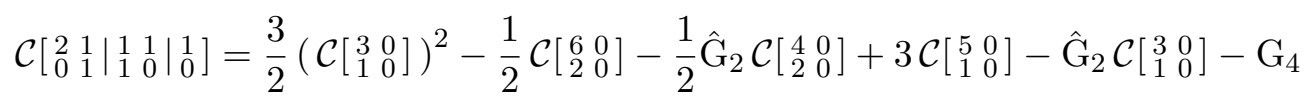

$$
\begin{aligned}
& \mathcal{C}\left[\begin{array}{llll}
1 & 1 & 1 & 1 \\
1 & 0 & 1 & 0 \\
1 & 0
\end{array}\right]=-2\left(\mathcal{C}\left[\begin{array}{ll}
3 & 0 \\
1 & 0
\end{array}\right]\right)^{2}+2 \mathcal{C}\left[\begin{array}{ll}
6 & 0 \\
2 & 0
\end{array}\right]+\hat{\mathrm{G}}_{2} \mathcal{C}\left[\begin{array}{ll}
4 & 0 \\
2 & 0
\end{array}\right]-6 \mathcal{C}\left[\begin{array}{ll}
5 & 0 \\
1 & 0
\end{array}\right]+2 \hat{\mathrm{G}}_{2} \mathcal{C}\left[\begin{array}{ll}
3 & 0 \\
1 & 0
\end{array}\right]+2 \mathrm{G}_{4} \\
& \mathcal{C}_{\text {tet }}=4\left(\mathcal{C}\left[\begin{array}{ll}
3 & 0 \\
1 & 0
\end{array}\right]\right)^{2}-2 \mathcal{C}\left[\begin{array}{ll}
6 & 0 \\
2 & 0
\end{array}\right]-2 \hat{\mathrm{G}}_{2} \mathcal{C}\left[\begin{array}{ll}
4 & 0 \\
2 & 0
\end{array}\right]+12 \mathcal{C}\left[\begin{array}{ll}
5 & 0 \\
1 & 0
\end{array}\right]-4 \hat{\mathrm{G}}_{2} \mathcal{C}\left[\begin{array}{ll}
3 & 0 \\
1 & 0
\end{array}\right]-4 \mathrm{G}_{4} \text {. }
\end{aligned}
$$

Furthermore, the identities among $\mathcal{C}\left[\begin{array}{lll}a & b & c \\ 1 & 1 & 0\end{array}\right]$ in appendix A.1 and holomorphic subgraph reduction as in (3.25) reduce all the dihedral modular graph forms in $H_{1234}^{(4,0)}\left[G_{i j} G_{k l}\right]$ to the simplest class $\mathcal{C}\left[\begin{array}{ll}a & 0 \\ b & 0\end{array}\right]$ of lattice sums. For instance,

$$
\begin{aligned}
H_{1234}^{(4,0)}\left[G_{12}^{2}\right] & =\mathrm{G}_{4} \mathrm{E}_{2}-\hat{\mathrm{G}}_{2} \mathcal{C}\left[\begin{array}{ll}
4 & 0 \\
2 & 0
\end{array}\right]-8 \mathcal{C}\left[\begin{array}{ll}
5 & 0 \\
1 & 0
\end{array}\right]+2 \hat{\mathrm{G}}_{2} \mathcal{C}\left[\begin{array}{ll}
3 & 0 \\
1 & 0
\end{array}\right]+2 \mathrm{G}_{4} \\
H_{1234}^{(4,0)}\left[G_{12} G_{34}\right] & =\hat{\mathrm{G}}_{2} \mathcal{C}\left[\begin{array}{ll}
4 & 0 \\
2 & 0
\end{array}\right]-6 \mathcal{C}\left[\begin{array}{ll}
5 & 0 \\
1 & 0
\end{array}\right]+2 \hat{\mathrm{G}}_{2} \mathcal{C}\left[\begin{array}{ll}
3 & 0 \\
1
\end{array}\right]+2 \mathrm{G}_{4} .
\end{aligned}
$$

Once this simplification is performed for all heterotic graph forms in (4.22), the remaining $H_{1234}^{(4,0)}\left[G_{i j} G_{k l}\right]$ are found to be related to (4.26) and (4.27) via

$$
\begin{aligned}
H_{1234}^{(4,0)}\left[G_{13}^{2}\right] & =3 \mathrm{G}_{4} \mathrm{E}_{2}-2 H_{1234}^{(4,0)}\left[G_{12}^{2}\right] \\
H_{1234}^{(4,0)}\left[G_{13} G_{24}\right] & =-2 H_{1234}^{(4,0)}\left[G_{12} G_{34}\right] \\
H_{1234}^{(4,0)}\left[G_{13} G_{12}\right] & =-\frac{1}{2} H_{1234}^{(4,0)}\left[G_{12} G_{34}\right] \\
H_{1234}^{(4,0)}\left[G_{12} G_{23}\right] & =H_{1234}^{(4,0)}\left[G_{12} G_{34}\right] .
\end{aligned}
$$

Furthermore, the contributions of $\hat{\mathrm{G}}_{2} \mathcal{C}\left[\begin{array}{ll}3 & 0 \\ 1 & 0\end{array}\right]$ and $\mathrm{G}_{4}$ turn out to cancel when assembling the complete $\alpha^{\prime 2}$ order of $\mathcal{I}_{1234}^{(4,0)}$, see (4.33) below.

We have also evaluated the $\alpha^{\prime 3}$-order of the integral $\mathcal{I}_{1234}^{(2,0)}$, see appendix B.1 for the occurring modular graph forms and appendix B.2 for their simplifications. Their specific combination selected by applying momentum conservation to $\mathcal{I}_{1234}^{(2,0)}$ will be given below.

\subsubsection{Checking and assembling the results}

The symmetry properties of the $V_{w}$ functions [25]

$$
\begin{aligned}
& V_{2}(1,2,3,4)+V_{2}(1,3,4,2)+V_{2}(1,4,2,3)=0 \\
& V_{4}(1,2,3,4)+V_{4}(1,3,4,2)+V_{4}(1,4,2,3)=3 \mathrm{G}_{4}
\end{aligned}
$$

impose the following constraints on heterotic graph forms

$$
\begin{aligned}
& H_{1234}^{(2,0)}[\ldots]+H_{1342}^{(2,0)}[\ldots]+H_{1423}^{(2,0)}[\ldots]=0 \\
& H_{1234}^{(4,0)}[\ldots]+H_{1342}^{(4,0)}[\ldots]+H_{1423}^{(4,0)}[\ldots]=3 \mathrm{G}_{4} H_{1234}^{(0,0)}[\ldots],
\end{aligned}
$$


where the ellipses represent arbitrary monomials in $G_{i j}$ (the same ones in each term of the respective equation), and all of our results for $H_{1234}^{(w, 0)}[\ldots]$ have been checked to satisfy these consistency conditions. Examples for the modular graph functions on the right-hand side of (4.30) include

$$
H_{1234}^{(0,0)}[\emptyset]=1, \quad H_{1234}^{(0,0)}\left[G_{i j}\right]=0, \quad H_{1234}^{(0,0)}\left[G_{i j}^{2}\right]=\mathrm{E}_{2} .
$$

With the results for the planar heterotic graph forms $H_{1234}^{(2,0)}[\ldots]$ at orders $\alpha^{\prime \leq 3}$ and $H_{1234}^{(4,0)}[\ldots]$ at orders $\alpha^{\prime \leq 2}$, we arrive at the low-energy expansions (cf. (4.13))

$$
\begin{aligned}
\mathcal{I}_{1234}^{(2,0)}\left(s_{i j}, \tau\right)= & 6 s_{13} \mathcal{C}\left[\begin{array}{ll}
3 & 0 \\
1 & 0
\end{array}\right]+2\left(s_{13}^{2}+2 s_{12} s_{23}\right) \mathcal{C}\left[\begin{array}{ll}
4 & 0 \\
2 & 0
\end{array}\right] \\
& +4 s_{13}\left(s_{13}^{2}-s_{12} s_{23}\right)\left(3 \mathcal{C}\left[\begin{array}{lll}
3 & 1 & 1 \\
1 & 1
\end{array}\right]-4 \mathcal{C}\left[\begin{array}{ll}
5 & 0 \\
3 & 0
\end{array}\right]+3 \mathrm{E}_{2} \mathcal{C}\left[\begin{array}{ll}
3 & 0 \\
1 & 0
\end{array}\right]\right)+\mathcal{O}\left(\alpha^{\prime 4}\right) \\
\mathcal{I}_{1234}^{(4,0)}\left(s_{i j}, \tau\right)= & \mathrm{G}_{4}+6 s_{13}\left(\mathrm{G}_{4}+\hat{\mathrm{G}}_{2} \mathcal{C}\left[\begin{array}{ll}
3 & 0 \\
1 & 0
\end{array}\right]\right)+2\left(s_{13}^{2}-s_{12} s_{23}\right) \mathrm{G}_{4} \mathrm{E}_{2} \\
& +2\left(s_{13}^{2}+2 s_{12} s_{23}\right)\left(\mathcal{C}\left[\begin{array}{ll}
5 & 0 \\
1 & 0
\end{array}\right]+\hat{\mathrm{G}}_{2} \mathcal{C}\left[\begin{array}{ll}
4 & 0 \\
2 & 0
\end{array}\right]\right)+\mathcal{O}\left(\alpha^{\prime 3}\right)
\end{aligned}
$$

after applying the Mandelstam identities (2.41). One can use the equivalent form of $(3.34)^{16}$

$$
\begin{aligned}
& \mathcal{C}\left[\begin{array}{ll}
3 & 0 \\
1 & 0
\end{array}\right]=\frac{\pi \nabla \mathrm{E}_{2}}{2(\operatorname{Im} \tau)^{2}}, \quad \mathcal{C}\left[\begin{array}{ll}
4 & 0 \\
2 & 0
\end{array}\right]=\frac{\pi \nabla \mathrm{E}_{3}}{3(\operatorname{Im} \tau)^{2}}, \quad \mathcal{C}\left[\begin{array}{ll}
5 & 0 \\
1 & 0
\end{array}\right]=\frac{(\pi \nabla)^{2} \mathrm{E}_{3}}{12(\operatorname{Im} \tau)^{4}} \\
& \mathcal{C}\left[\begin{array}{ll}
5 & 0 \\
3 & 0
\end{array}\right]=\frac{\pi \nabla \mathrm{E}_{4}}{4(\operatorname{Im} \tau)^{2}}, \quad \mathcal{C}\left[\begin{array}{lll}
3 & 1 & 1 \\
1 & 1 & 1
\end{array}\right]-\frac{8}{5} \mathcal{C}\left[\begin{array}{ll}
5 & 0 \\
3 & 0
\end{array}\right]=\frac{\pi \nabla \mathrm{E}_{2,2}}{(\operatorname{Im} \tau)^{2}}
\end{aligned}
$$

to express these expansions via Cauchy-Riemann derivatives of modular invariants $\mathrm{E}_{\ldots}$,

$$
\begin{aligned}
(\operatorname{Im} \tau)^{2} \mathcal{I}_{1234}^{(2,0)}\left(s_{i j}, \tau\right)= & 3 s_{13} \pi \nabla \mathrm{E}_{2}+\frac{2}{3}\left(s_{13}^{2}+2 s_{12} s_{23}\right) \pi \nabla \mathrm{E}_{3} \\
& +s_{13}\left(s_{13}^{2}-s_{12} s_{23}\right)\left(\frac{4}{5} \pi \nabla \mathrm{E}_{4}+12 \pi \nabla \mathrm{E}_{2,2}+6 \mathrm{E}_{2} \pi \nabla \mathrm{E}_{2}\right)+\mathcal{O}\left(\alpha^{\prime 4}\right) \\
\mathcal{I}_{1234}^{(4,0)}\left(s_{i j}, \tau\right)= & \mathrm{G}_{4}+3 s_{13}\left(2 \mathrm{G}_{4}+\frac{\hat{\mathrm{G}}_{2} \pi \nabla \mathrm{E}_{2}}{(\operatorname{Im} \tau)^{2}}\right)+2\left(s_{13}^{2}-s_{12} s_{23}\right) \mathrm{G}_{4} \mathrm{E}_{2} \\
& +\left(s_{13}^{2}+2 s_{12} s_{23}\right)\left(\frac{(\pi \nabla)^{2} \mathrm{E}_{3}}{6(\operatorname{Im} \tau)^{4}}+\frac{2 \hat{\mathrm{G}}_{2} \pi \nabla \mathrm{E}_{3}}{3(\operatorname{Im} \tau)^{2}}\right)+\mathcal{O}\left(\alpha^{\prime 3}\right)
\end{aligned}
$$

see (3.36) for the definition of $\mathrm{E}_{2,2}$ and (3.9) for the analogous expansion of $\mathcal{I}^{(0,0)}$.

\subsection{Non-planar heterotic graph forms}

We will now adapt the strategy of the previous section to the low-energy expansion of the Koba-Nielsen integrals $\mathcal{I}_{12 \mid 34}^{(w, 0)}$ in the double-trace sector. The non-planar heterotic graph forms (4.14) in the $\alpha^{\prime}$-expansion take the most compact form when we compute them from the Fourier expansion $(4.9)$ of $V_{2}(i, j)$. However, some of the resulting lattice sums turn out to be conditionally convergent or even divergent. We define a regularization scheme by demanding that (4.9) and the alternative Fourier expansion due to insertion of (4.2) into

$$
V_{2}(i, j)=2 f_{i j}^{(2)}-\left(f_{i j}^{(1)}\right)^{2}
$$

\footnotetext{
${ }^{16}$ We have also employed $\pi \nabla C_{2,1,1}=2(\operatorname{Im} \tau)^{2}\left(\mathcal{C}\left[\begin{array}{lll}3 & 1 & 1 \\ 1 & 1 & 1\end{array}\right]+\mathcal{C}\left[\begin{array}{lll}2 & 2 & 1 \\ 2 & 0 & 1\end{array}\right]\right)$ and $\mathcal{C}\left[\begin{array}{lll}3 & 1 & 1 \\ 1 & 1 & 1\end{array}\right]+2 \mathcal{C}\left[\begin{array}{lll}2 & 2 & 1 \\ 2 & 0 & 1\end{array}\right]-2 \mathcal{C}\left[\begin{array}{ll}5 & 0 \\ 3 & 0\end{array}\right]=0$ in deriving (4.34).
} 


\begin{tabular}{|cc|}
\hline Order & Inequivalent non-planar heterotic graph forms \\
\hline$\alpha^{\prime 0}$ & $H_{12 \mid 34}^{(w, 0)}[\emptyset]$ \\
$\alpha^{\prime 1}$ & $H_{12 \mid 34}^{(w, 0)}\left[G_{12}\right], H_{12 \mid 34}^{(w, 0)}\left[G_{13}\right]$ \\
$\alpha^{\prime 2}$ & $H_{12 \mid 34}^{(w, 0)}\left[G_{12}^{2}\right], H_{12 \mid 34}^{(w, 0)}\left[G_{12} G_{34}\right], H_{12 \mid 34}^{(w, 0)}\left[G_{13} G_{24}\right]$ \\
& $H_{12 \mid 34}^{(w, 0)}\left[G_{13}^{2}\right], H_{12 \mid 34}^{(w, 0)}\left[G_{12} G_{13}\right], H_{12 \mid 34}^{(w, 0)}\left[G_{13} G_{23}\right]$ \\
$\alpha^{\prime 3}$ & $H_{12 \mid 34}^{(w, 0)}\left[G_{12}^{3}\right], H_{12 \mid 34}^{(w, 0)}\left[G_{12}^{2} G_{13}\right], H_{12 \mid 34}^{(w, 0)}\left[G_{12} G_{13} G_{34}\right], H_{12 \mid 34}^{(w, 0)}\left[G_{12} G_{13} G_{24}\right]$ \\
& $H_{12 \mid 34}^{(w, 0)}\left[G_{13}^{3}\right], H_{12 \mid 34}^{(w, 0)}\left[G_{12} G_{13}^{2}\right], H_{12 \mid 34}^{(w, 0)}\left[G_{12} G_{13} G_{14}\right], H_{12 \mid 34}^{(w, 0)}\left[G_{13} G_{14} G_{34}\right]$ \\
& $H_{12 \mid 34}^{(w, 0)}\left[G_{12}^{2} G_{34}\right], H_{12 \mid 34}^{(w, 0)}\left[G_{13}^{2} G_{14}\right], H_{12 \mid 34}^{(w, 0)}\left[G_{13}^{2} G_{24}\right], H_{12 \mid 34}^{(w, 0)}\left[G_{13} G_{14} G_{23}\right]$ \\
\hline
\end{tabular}

Table 2. Inequivalent non-planar heterotic graph forms with respect to the symmetries $H_{12 \mid 34}^{(w, 0)}=H_{21 \mid 34}^{(w, 0)}=H_{34 \mid 12}^{(w, 0)}$.

yield the same expression for each heterotic graph form. Again, we exploit the symmetries

$$
\mathcal{I}_{12 \mid 34}^{(w, 0)}=\mathcal{I}_{21 \mid 34}^{(w, 0)}=\mathcal{I}_{34 \mid 12}^{(w, 0)}
$$

of the integrals at each order in $\alpha^{\prime}$ to reduce the number of heterotic graph forms that need to be calculated independently, see table 2 .

\subsubsection{Leading orders $\alpha^{\prime 0}$ and $\alpha^{\prime 1}$}

In the absence of Green functions in the integrand, only the zero mode $\hat{\mathrm{G}}_{2}$ of the Fourier expansion (4.9) of $V_{2}(i, j)$ contributes,

$$
H_{12 \mid 34}^{(2,0)}[\emptyset]=2 \hat{\mathrm{G}}_{2}, \quad H_{12 \mid 34}^{(4,0)}[\emptyset]=\hat{\mathrm{G}}_{2}^{2} .
$$

At first order in $\alpha^{\prime}$, the representation $(4.9)$ of $V_{2}(i, j)$ yields conditionally convergent lattice sums

$$
H_{12 \mid 34}^{(2,0)}\left[G_{12}\right]=-\mathcal{C}_{\text {reg }}\left[\begin{array}{ll}
2 & 0 \\
0 & 0
\end{array}\right], \quad H_{12 \mid 34}^{(4,0)}\left[G_{12}\right]=-\hat{\mathrm{G}}_{2} \mathcal{C}_{\mathrm{reg}}\left[\begin{array}{ll}
2 & 0 \\
0 & 0
\end{array}\right]
$$

The regularized value of $\mathcal{C}_{\text {reg }}\left[\begin{array}{ll}2 & 0 \\ 0 & 0\end{array}\right]$ and similar lattice sums will be determined by repeating the above calculations with the representation $(4.37)$ of $V_{2}(i, j)$ in terms of $f^{(w)}$ functions,

$$
H_{12 \mid 34}^{(2,0)}[\emptyset]=2 \mathcal{C}_{\text {reg }}\left[\begin{array}{ll}
2 & 0 \\
0 & 0
\end{array}\right], \quad H_{12 \mid 34}^{(2,0)}\left[G_{12}\right]=-2 \mathcal{C}\left[\begin{array}{ll}
3 & 0 \\
1 & 0
\end{array}\right]-\mathcal{C}_{\text {reg }}\left[\begin{array}{lll}
1 & 1 & 1 \\
0 & 0 & 1
\end{array}\right]
$$

By imposing these results to match (4.39) and (4.40), we can determine both $\mathcal{C}_{\text {reg }}\left[\begin{array}{ll}2 & 0 \\ 0 & 0\end{array}\right]$ and another regularized value $\mathcal{C}_{\text {reg }}\left[\begin{array}{lll}1 & 1 & 1 \\ 0 & 0 & 1\end{array}\right]$ relevant to a later step, ${ }^{17}$

$$
\mathcal{C}_{\text {reg }}\left[\begin{array}{ll}
2 & 0 \\
0 & 0
\end{array}\right]=\hat{\mathrm{G}}_{2}, \quad \mathcal{C}_{\mathrm{reg}}\left[\begin{array}{lll}
1 & 1 & 1 \\
0 & 0 & 1
\end{array}\right]=\hat{\mathrm{G}}_{2}-2 \mathcal{C}\left[\begin{array}{ll}
3 & 0 \\
1 & 0
\end{array}\right],
$$

\footnotetext{
${ }^{17}$ Note that the expressions in (4.42) cannot be reproduced from an extension of momentum conservation (say $\left.0=\mathcal{C}_{\text {reg }}\left[\begin{array}{lll}1 & 1 & 1 \\ 0 & 0 & 1\end{array}\right]+2 \mathcal{C}_{\text {reg }}\left[\begin{array}{lll}2 & 1 & 0 \\ 1 & 0 & 0\end{array}\right]\right)$ and (3.20) to regularized values.
} 
see also [23] for the first result. A more general class of identities between regularized values can be generated by integrating the lattice sum $\sum_{p \neq 0} \frac{e^{2 \pi i\left\langle p, z_{i j}\right\rangle}}{p^{a} \bar{p}^{b}}$ against the two representations (4.9) and (4.37) of $V_{2}(i, j),{ }^{18}$

$$
\mathcal{C}_{\text {reg }}\left[\begin{array}{lll}
1 & 1 & a \\
0 & 0 & b
\end{array}\right]=\mathcal{C}_{\text {reg }}\left[\begin{array}{ll}
a+1 & 0 \\
b-1 & 0
\end{array}\right]-2 \mathcal{C}\left[\begin{array}{rr}
a+2 & 0 \\
b & 0
\end{array}\right]
$$

where $\mathcal{C}_{\text {reg }}\left[\begin{array}{l}A \\ B\end{array}\right]:=\mathcal{C}\left[\begin{array}{l}A \\ B\end{array}\right]$ whenever the entries of $A$ and $B$ yield absolutely convergent sums. With the above regularized values, the inequivalent heterotic graph forms at the first order in $\alpha^{\prime}$ read

$$
\begin{array}{ll}
H_{12 \mid 34}^{(2,0)}\left[G_{12}\right]=-\hat{\mathrm{G}}_{2}, & H_{12 \mid 34}^{(2,0)}\left[G_{13}\right]=0 \\
H_{12 \mid 34}^{(4,0)}\left[G_{12}\right]=-\hat{\mathrm{G}}_{2}^{2}, & H_{12 \mid 34}^{(4,0)}\left[G_{13}\right]=0 .
\end{array}
$$

\subsubsection{Subleading orders $\alpha^{\prime 2}, \alpha^{3}$ and beyond}

At the second order in $\alpha^{\prime}$, we exploit the expressions for $\mathcal{C}_{\text {reg }}\left[\begin{array}{ll}2 & 0 \\ 0 & 0\end{array}\right]$ and $\mathcal{C}_{\text {reg }}\left[\begin{array}{lll}1 & 1 & 1 \\ 0 & 0 & 1\end{array}\right]$ in $(4.42)$ to determine the inequivalent heterotic graph forms

$$
\begin{aligned}
H_{12 \mid 34}^{(2,0)}\left[G_{12}^{2}\right] & =2 \hat{\mathrm{G}}_{2} \mathrm{E}_{2}+2 \hat{\mathrm{G}}_{2}-4 \mathcal{C}\left[\begin{array}{ll}
3 & 0 \\
1 & 0
\end{array}\right] \\
H_{12 \mid 34}^{(2,0)}\left[G_{13}^{2}\right] & =2 \hat{\mathrm{G}}_{2} \mathrm{E}_{2} \\
H_{12 \mid 34}^{(2,0)}\left[G_{13} G_{23}\right] & =-\mathcal{C}\left[\begin{array}{ll}
3 & 0 \\
1 & 0
\end{array}\right] \\
H_{12 \mid 34}^{(2,0)}\left[G_{12} G_{13}\right] & =H_{12 \mid 34}^{(2,0)}\left[G_{12} G_{34}\right]=H_{12 \mid 34}^{(2,0)}\left[G_{13} G_{24}\right]=0
\end{aligned}
$$

and

$$
\begin{aligned}
H_{12 \mid 34}^{(4,0)}\left[G_{12}^{2}\right] & =\hat{\mathrm{G}}_{2}^{2} \mathrm{E}_{2}+2 \hat{\mathrm{G}}_{2}^{2}-4 \hat{\mathrm{G}}_{2} \mathcal{C}\left[\begin{array}{ll}
3 & 0 \\
1 & 0
\end{array}\right], & H_{12 \mid 34}^{(4,0)}\left[G_{12} G_{13}\right] & =0 \\
H_{12 \mid 34}^{(4,0)}\left[G_{13} G_{24}\right] & =\mathrm{G}_{4}, & H_{12 \mid 34}^{(4,0)}\left[G_{13}^{2}\right] & =\hat{\mathrm{G}}_{2}^{2} \mathrm{E}_{2} \\
H_{12 \mid 34}^{(4,0)}\left[G_{12} G_{34}\right] & =\hat{\mathrm{G}}_{2}^{2}, & H_{12 \mid 34}^{(4,0)}\left[G_{13} G_{23}\right] & =-\hat{\mathrm{G}}_{2} \mathcal{C}\left[\begin{array}{ll}
3 & 0 \\
1 & 0
\end{array}\right] .
\end{aligned}
$$

On top of (4.43), the third order in $\alpha^{\prime}$ involves the regularized value

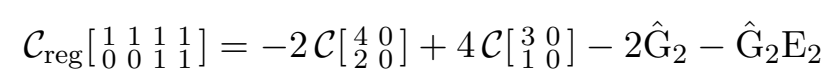

which has been inferred by evaluating $H_{12 \mid 34}^{(2,0)}\left[G_{12}^{2}\right]$ with both representations (4.9) and (4.37) of $V_{2}(i, j)$. The inequivalent heterotic graph forms at order $\alpha^{\prime 3}$ from table 2 are given in appendix $\mathrm{C}$. The four-point gauge amplitude only requires a specific combination of them that simplifies and is given below.

For higher orders in $\alpha^{\prime}$, we speculate that the above method can be used to assign a regularized value to all the conditionally convergent or divergent sums in the nonplanar heterotic graph forms: the idea is to integrate suitably chosen lattice sums (such as $\sum_{p \neq 0} \frac{e^{2 \pi i\left\langle p, z_{i j}\right\rangle}}{p^{a} \bar{p}^{b}}$ in case of $\left.(4.43)\right)$ against $V_{2}(i, j)$ or $V_{2}(i, j) V_{2}(k, l)$ and equate the results that arise from different use of (4.9) and (4.37). In particular, this technique has been checked to yield unique regularized values for the most challenging contributions at order $\alpha^{\prime 4}$.

\footnotetext{
${ }^{18}$ Note that (4.43) is inconsistent with a naive extension of the holomorphic subgraph reduction (3.22) to $a_{+}=a_{-}=1$ and regularized values.
} 


\subsubsection{Assembling the results}

With the results for the non-planar heterotic graph forms at order $\alpha^{\prime \leq 3}$, the low-energy expansions of the integrals $\mathcal{I}_{12 \mid 34}^{(w, 0)}$ are found to be

$$
\begin{aligned}
\mathcal{I}_{12 \mid 34}^{(2,0)}= & 2 \hat{\mathrm{G}}_{2}-2 s_{12} \hat{\mathrm{G}}_{2}+2 s_{12}^{2}\left(\hat{\mathrm{G}}_{2}\left(1+2 \mathrm{E}_{2}\right)-\frac{\pi \nabla \mathrm{E}_{2}}{(\operatorname{Im} \tau)^{2}}\right)-2 s_{13} s_{23}\left(2 \hat{\mathrm{G}}_{2} \mathrm{E}_{2}+\frac{\pi \nabla \mathrm{E}_{2}}{(\operatorname{Im} \tau)^{2}}\right) \\
& +2 s_{12}^{3}\left(\frac{\pi \nabla \mathrm{E}_{2}}{(\operatorname{Im} \tau)^{2}}-\frac{2}{3} \frac{\pi \nabla \mathrm{E}_{3}}{(\operatorname{Im} \tau)^{2}}-\left(1+2 \mathrm{E}_{2}\right) \hat{\mathrm{G}}_{2}\right) \\
& +2 s_{12} s_{13} s_{23}\left(\left(2 \mathrm{E}_{2}+5 \mathrm{E}_{3}+\zeta_{3}\right) \hat{\mathrm{G}}_{2}+\frac{\pi \nabla \mathrm{E}_{2}}{(\operatorname{Im} \tau)^{2}}-\frac{2}{3} \frac{\pi \nabla \mathrm{E}_{3}}{(\operatorname{Im} \tau)^{2}}\right)+\mathcal{O}\left(\alpha^{\prime 4}\right) \\
\mathcal{I}_{12 \mid 34}^{(4,0)}= & \hat{\mathrm{G}}_{2}^{2}-2 s_{12} \hat{\mathrm{G}}_{2}^{2} \\
& +s_{12}^{2}\left(\mathrm{G}_{4}+\hat{\mathrm{G}}_{2}^{2}\left(3+2 \mathrm{E}_{2}\right)-\frac{2 \hat{\mathrm{G}}_{2} \pi \nabla \mathrm{E}_{2}}{(\operatorname{Im} \tau)^{2}}\right)-2 s_{13} s_{23}\left(\mathrm{G}_{4}+\hat{\mathrm{G}}_{2}^{2} \mathrm{E}_{2}+\frac{\hat{\mathrm{G}}_{2} \pi \nabla \mathrm{E}_{2}}{(\operatorname{Im} \tau)^{2}}\right) \\
& +s_{12} s_{13} s_{23}\left(\left(4 \mathrm{E}_{2}+5 \mathrm{E}_{3}+\zeta_{3}\right) \hat{\mathrm{G}}_{2}^{2}-2 \mathrm{G}_{4}+\frac{(\pi \nabla)^{2} \mathrm{E}_{3}}{(\operatorname{Im} \tau)^{4}}+\frac{\hat{\mathrm{G}}_{2} \pi \nabla \mathrm{E}_{2}}{(\operatorname{Im} \tau)^{2}}-\frac{4}{3} \frac{\hat{\mathrm{G}}_{2} \pi \nabla \mathrm{E}_{3}}{(\operatorname{Im} \tau)^{2}}\right) \\
& +s_{12}^{3}\left(-2 \mathrm{G}_{4}-4 \hat{\mathrm{G}}_{2}^{2}\left(1+\mathrm{E}_{2}\right)+\frac{(\pi \nabla)^{2} \mathrm{E}_{3}}{3(\operatorname{Im} \tau)^{4}}+\frac{4 \hat{\mathrm{G}}_{2} \pi \nabla \mathrm{E}_{2}}{(\operatorname{Im} \tau)^{2}}-\frac{4}{3} \frac{\hat{\mathrm{G}}_{2} \pi \nabla \mathrm{E}_{3}}{(\operatorname{Im} \tau)^{2}}\right)+\mathcal{O}\left(\alpha^{\prime 4}\right)
\end{aligned}
$$

after applying momentum conservation. Similar to the representations (4.35) and (4.36) of the planar integrals, we have used the substitutions (4.34). Together with the planar results and the expression (3.9) for $\mathcal{I}^{(0,0)},(4.48)$ and (4.49) complete the ingredients for the $\tau$ integrand (2.39) of the heterotic-string amplitude.

Note that (4.48) and (4.49) have been reproduced from the alternative method in appendix D.2, where the $\alpha^{\prime}$-expansion of $\mathcal{I}_{12 \mid 34}^{(w, 0)}$ is performed without any conditionally convergent or divergent sums in intermediate steps. This adds further validation for the assignment of regularized values described above.

\subsection{Uniform transcendentality and $\alpha^{\prime}$-expansions}

As mentioned in the introduction, one of the remarkable features of type-II amplitudes is that they exhibit so-called uniform transcendentality at each order in $\alpha^{\prime}$. In this section, we will study the transcendentality properties of the heterotic string by restricting to the salient points that require a rewriting of the basis integrals $\mathcal{I}_{\ldots}^{(w, 0)}$; additional details can be found in appendix D.

In analogy with the superstring, we associate transcendental weights to the various objects appearing in the low-energy expansion of the heterotic integrals over the punctures as follows. The Eisenstein series $\mathrm{G}_{k}$ and $\mathrm{E}_{k}$ as well as $\zeta_{k}$ are assigned transcendental weight $k$, i.e. $\pi$ has transcendental weight one, whereas $\tau$ and $\nabla$ have transcendental weight zero. Accordingly, one finds transcendental weight one for both $\pi \nabla$ and $y=\pi \operatorname{Im} \tau$, i.e. weight $k+p$ for $(\pi \nabla)^{p} \mathrm{E}_{k}$, and weight $4+p$ for $(\pi \nabla)^{p} \mathrm{E}_{2,2}$. A more general definition of transcendental weight in terms of iterated integrals is given in appendix $\mathrm{D}$, but the assignment above suffices for the discussion of this section. 
Inspecting the $\alpha^{\prime}$-expansions of the planar single-trace integrals $\mathcal{I}^{(0,0)}$ and $\mathcal{I}_{1234}^{(2,0)}$ of (3.9) and (4.35), one sees that their $k^{\text {th }}$ order consistently involves modular graph forms of weight $k$ and $k+2$, respectively. Thus, these two integrals are referred to as uniformly transcendental.

By contrast, $\mathcal{I}_{1234}^{(4,0)}$ in (4.36) violates uniform transcendentality since the same type of transcendental object appears at different orders in the $\alpha^{\prime}$-expansion. For instance, $\mathrm{G}_{4}$ of transcendentality four appears with $1+6 s_{13}+\ldots$ and thus at different orders in $\alpha^{\prime}$. Similarly, the integrals $\mathcal{I}_{12 \mid 34}^{(w, 0)}$ in (4.48) and (4.49) from the double-trace sector violate uniform transcendentality. This can for instance be seen from the terms $\sim\left(1+2 \mathrm{E}_{2}\right)$ along with $s_{12}^{2}$ in $\mathcal{I}_{12 \mid 34}^{(2,0)}$ and $\sim\left(3+2 \mathrm{E}_{2}\right)$ along with $s_{12}^{2}$ in $\mathcal{I}_{12 \mid 34}^{(4,0)}$, respectively.

This violation of uniform transcendentality can be traced back to the following phenomenon. For the planar integral $\mathcal{I}_{1234}^{(4,0)}$ we see from $(4.13),(4.12)$ and $(4.15)$ that there is a leading contribution with a closed cycle of the form $f_{12}^{(1)} f_{23}^{(1)} f_{34}^{(1)} f_{41}^{(1)}$. This cycle exhibits purely holomorphic modular weight $(4,0)$ and is thus amenable to holomorphic subgraph reduction discussed in section 3.2.2. As is evident from the explicit examples in (3.24), the reduction produces various terms of different transcendentality: $\mathcal{C}\left[\begin{array}{ll}5 & 0 \\ 1 & 0\end{array}\right]$ carries transcendental weight 5 by (4.34) whereas $\mathrm{G}_{4}$ carries weight 4 . Moreover, the formula (3.22) for dihedral holomorphic subgraphs generically produces explicit factors of $\hat{\mathrm{G}}_{2}=\mathrm{G}_{2}-\frac{\pi}{\operatorname{Im} \tau}$ which are clearly not of uniform transcendental weight. We therefore expect that all closed cycles $f_{12}^{(1)} f_{23}^{(1)} \ldots f_{k 1}^{(1)}$ in the $n$-point integrand break uniform transcendentality, including those with $k=n$. This is in marked contrast with the genus-zero situation where only closed subcycles $\left(z_{12} z_{23} \ldots z_{k 1}\right)^{-1}$ in the integrand with $k \leq n-2$ violate uniform transcendentality $[40,71-73] .{ }^{19}$ The subcycles $f_{12}^{(1)} f_{21}^{(1)}$ in the integrands of the non-uniformly transcendental integrals $\mathcal{I}_{12 \mid 34}^{(2,0)}$ and $\mathcal{I}_{12 \mid 34}^{(4,0)}$ in the non-planar sector confirm the general expectation.

At genus zero, any non-uniformly transcendental disk or sphere integral over $n$ punctures can be expanded in a basis of uniformly transcendental integrals, see [47] for a general argument and $[40,73,74]$ for examples. This basis, known as Parke-Taylor basis, consists of $(n-3)$ ! elements $[74,75]$ and spans the twisted cohomology defined by the Koba-Nielsen factor made out of $\left|z_{i j}\right|^{-s_{i j}}[47]$.

At genus one, the classification of integration-by-parts inequivalent half-integrands i.e. chiral halves for torus integrands - is an open problem. While genus-one correlators of the open superstring exclude a variety of worldsheet functions by maximal supersymmetry [55, 56], Kac-Moody correlators such as (2.37) give a more accurate picture of the problem: it remains to find a minimal set of integrals that span all the above $\mathcal{I}_{\ldots}^{(w, 0)}$ (and possibly other torus integrals from different massless one-loop closed-string amplitudes)

\footnotetext{
${ }^{19}$ This point can be illustrated by considering a four-point integral at genus zero over closed subcycles with an integrand of the form $\left(z_{12} z_{21}\right)^{-1}\left(z_{34} z_{43}\right)^{-1}$. Integrating by parts in $z$ leads to the cyclic factor $\left(z_{12} z_{23} z_{34} z_{41}\right)^{-1}$ subtending all four punctures that is called a Parke-Taylor factor (see section 5$)$. The integration by parts also generates the rational factor $\frac{s_{23}}{1+s_{12}}$ in Mandelstam invariants that mixes different orders in $\alpha^{\prime}$. Genus-zero integrals over Parke-Taylor factors subtending all the punctures are known to be uniformly transcendental (which is for instance evident from their representation in terms of the Drinfeld associator [4]). Hence, the original subcycle expression with $\left(z_{12} z_{21}\right)^{-1}\left(z_{34} z_{43}\right)^{-1}$ must violate uniform transcendentality.
} 
via integration by parts. These equivalence classes are again referred to as twisted cohomologies, where the twist is defined by the Koba-Nielsen factor $\exp \left(\sum_{i<j} s_{i j} G_{i j}\right)$ with the Green function in (2.15).

Therefore we shall now re-express the planar and non-planar integrands in a basis of uniformly transcendental integrals, hoping that this will also shed light on the question of a basis for twisted cohomologies at genus one. We present below candidate basis elements $\widehat{\mathcal{I}}^{(w, 0)}$ of conjectured uniform transcendentality that appear suitable for the four-current correlator (2.37). Our explicit expressions at leading orders in $\alpha^{\prime}$ and their different modular weights can be used to exclude relations among the $\widehat{\mathcal{I}}_{\ldots}^{(w, 0)}$. However, it is beyond the scope of this work to arrive at a reliable prediction for the basis dimension of uniformtranscendentality integrals at four points.

In the relation between the new quantities $\widehat{\mathcal{I}}_{\ldots}^{(w, 0)}$ and the genus-one integrals $\mathcal{I}_{1234}^{(4,0)}$, $\mathcal{I}_{12 \mid 34}^{(2,0)}$ and $\mathcal{I}_{12 \mid 34}^{(4,0)}$ all terms that break uniform transcendentality are contained in simple explicit coefficients like $\hat{\mathrm{G}}_{2}$ or $\left(1+s_{12}\right)^{-1}$. The manipulations necessary to arrive at the $\widehat{\mathcal{I}}_{\ldots}^{(w, 0)}$ are given in detail in appendices D.1 and D.2 and driven by integration by parts, resulting again in series in modular graph forms which

- bypass the need for holomorphic subgraph reduction

- avoid the conditionally convergent or divergent lattice sums caused by integration over $V_{2}(i, j)$, see section 4.3 for our regularization scheme.

Aspects of the computational complexity when using the $\mathcal{I}_{\ldots}^{(w, 0)}$ versus the $\widehat{\mathcal{I}}_{\ldots}^{(w, 0)}$ can be found in appendix D.3.

\subsubsection{The planar results in terms of uniform-transcendentality integrals}

As derived in appendix D.1, a decomposition of the single-trace part of the four-point gauge amplitude that exhibits uniform transcendentality is

$$
\left.M_{4}(\tau)\right|_{\operatorname{Tr}\left(t^{a_{1}} t^{a_{2}} t^{\left.a_{3} t^{a_{4}}\right)}\right.}=\mathrm{G}_{4}^{2} \widehat{\mathcal{I}}_{1234}^{(4,0)}+\mathrm{G}_{4}\left(\mathrm{G}_{4} \hat{\mathrm{G}}_{2}-\frac{7}{2} \mathrm{G}_{6}\right) \mathcal{I}_{1234}^{(2,0)}+\left(\frac{49}{6} \mathrm{G}_{6}^{2}-\frac{10}{3} \mathrm{G}_{4}^{3}\right) \mathcal{I}^{(0,0)} .
$$

In this expression we introduced the following combination of modular weight $(4,0)$

$$
\begin{aligned}
\widehat{\mathcal{I}}_{1234}^{(4,0)}\left(s_{i j}, \tau\right) & =\mathcal{I}_{1234}^{(4,0)}\left(s_{i j}, \tau\right)-\mathrm{G}_{4} \mathcal{I}_{1234}^{(0,0)}\left(s_{i j}, \tau\right)-\hat{\mathrm{G}}_{2} \mathcal{I}_{1234}^{(2,0)}\left(s_{i j}, \tau\right) \\
& =6 s_{13} \mathrm{G}_{4}+\left(s_{13}^{2}+2 s_{12} s_{23}\right) \frac{(\pi \nabla)^{2} \mathrm{E}_{3}}{6(\operatorname{Im} \tau)^{4}}+\mathcal{O}\left(\alpha^{\prime 3}\right)
\end{aligned}
$$

that manifestly respects uniform transcendentality to the order given. In (D.13), we provide a closed integral form of $\widehat{\mathcal{I}}_{1234}^{(4,0)}\left(s_{i j}, \tau\right)$ that we conjecture to be uniformly transcendental at every order in $\alpha^{\prime}$, with weight $k+3$ at the order of $\alpha^{\prime k}$. As argued above, $\mathcal{I}_{1234}^{(2,0)}$ and $\mathcal{I}^{(0,0)}$ are uniformly transcendental and all non-uniformly transcendental terms in the above way of writing the planar amplitude are in the coefficients of the basis integrals. The coefficient of $s_{13}$ in (4.51) may also be written as $6 \mathrm{G}_{4}=\frac{(\pi \nabla)^{2} \mathrm{E}_{2}}{(\operatorname{Im} \tau)^{4}}$ to highlight the parallel with the modular graph form $\frac{(\pi \nabla)^{2} \mathrm{E}_{3}}{6(\operatorname{Im} \tau)^{4}}$ at the subleading order $\alpha^{\prime 2}$. 
Note that the coefficient of $\mathcal{I}^{(0,0)}$ in (4.50) can be recognized as

$$
\frac{49}{6} \mathrm{G}_{6}^{2}-\frac{10}{3} \mathrm{G}_{4}^{3}=-\frac{128 \pi^{12}}{2025} \eta^{24} .
$$

At the level of the integrated amplitude (2.38), this cancels the factor of $\eta^{-24}$ due to the partition function. Hence, one can import the techniques of the type-II amplitude [10, 11] to perform the modular integrals $\int_{\mathcal{F}} \frac{\mathrm{d}^{2} \tau}{(\operatorname{Im} \tau)^{2}} \mathcal{I}^{(0,0)}$ arising from the terms $\sim \mathcal{I}^{(0,0)}$ in $(4.50)$ as we shall see in section 4.5 .

Similar to (4.52) the coefficient of $\mathcal{I}_{1234}^{(2,0)}$ in (4.50) exhibits a special relative factor in the combination $\mathrm{G}_{4} \mathrm{G}_{2}-\frac{7}{2} \mathrm{G}_{6}$ that can therefore be written as a $\tau$-derivative ${ }^{20}$

$$
\mathrm{G}_{4} \hat{\mathrm{G}}_{2}-\frac{7}{2} \mathrm{G}_{6}=-\frac{\pi}{\operatorname{Im} \tau} \mathrm{G}_{4}+\pi^{2} q \frac{\mathrm{dG}_{4}}{\mathrm{~d} q} .
$$

Hence, the only contribution $\sim q^{0}$ to (4.53) stems from the non-holomorphic term $-\frac{\pi}{\operatorname{Im} \tau} \mathrm{G}_{4}$.

\subsubsection{The non-planar results in terms of uniform-transcendentality integrals}

Similarly, we can also rewrite the non-planar part of the amplitude in terms of combinations that exhibit uniform transcendentality as follows

$$
\begin{aligned}
& \left.M_{4}(\tau)\right|_{\operatorname{Tr}\left(t^{a_{1}} t^{a_{2}}\right) \operatorname{Tr}\left(t^{a_{3}} t^{a_{4}}\right)}=\frac{\mathrm{G}_{4}^{2}\left[\widehat{\mathcal{I}}_{12 \mid 34}^{(4,0)}+s_{13}^{2}\left(\widehat{\mathcal{I}}_{1243}^{(4,0)}+\hat{\mathrm{G}}_{2} \mathcal{I}_{1243}^{(2,0)}\right)+s_{23}^{2}\left(\widehat{\mathcal{I}}_{1234}^{(4,0)}+\hat{\mathrm{G}}_{2} \mathcal{I}_{1234}^{(2,0)}\right)\right]}{\left(1+s_{12}\right)^{2}} \\
& +\left(\frac{\mathrm{G}_{4}^{2} \hat{\mathrm{G}}_{2}}{\left(1+s_{12}\right)^{2}}-\frac{7 \mathrm{G}_{4} \mathrm{G}_{6}}{2\left(1+s_{12}\right)}\right) \widehat{\mathcal{I}}_{12 \mid 34}^{(2,0)}+\left(\frac{\mathrm{G}_{4}^{2} \hat{\mathrm{G}}_{2}^{2}+\mathrm{G}_{4}^{3}\left(s_{13}^{2}+s_{23}^{2}\right)}{\left(1+s_{12}\right)^{2}}-7 \frac{\mathrm{G}_{4} \mathrm{G}_{6} \hat{\mathrm{G}}_{2}}{1+s_{12}}+\frac{5}{3} \mathrm{G}_{4}^{3}+\frac{49}{6} \mathrm{G}_{6}^{2}\right) \mathcal{I}^{(0,0)} .
\end{aligned}
$$

The details of the derivation of this result are given in appendix D.2. On top of a contribution from the planar integral given in (4.51), (4.54) contains the non-planar integrals of conjectured uniform transcendentality $\widehat{\mathcal{I}}_{12 \mid 34}^{(w, 0)}$ with modular weights $(w, 0)$ and leading orders

$$
\begin{aligned}
& \widehat{\mathcal{I}}_{12 \mid 34}^{(2,0)}=-2\left(s_{12}^{2}+s_{13} s_{23}\right) \frac{\pi \nabla \mathrm{E}_{2}}{(\operatorname{Im} \tau)^{2}}-4\left(s_{12}^{3}+s_{12} s_{13} s_{23}\right) \frac{\pi \nabla \mathrm{E}_{3}}{3(\operatorname{Im} \tau)^{2}}+\mathcal{O}\left(\alpha^{\prime 4}\right) \\
& \widehat{\mathcal{I}}_{12 \mid 34}^{(4,0)}=\left(s_{12}^{3}+3 s_{12} s_{13} s_{23}\right) \frac{(\pi \nabla)^{2} \mathrm{E}_{3}}{3(\operatorname{Im} \tau)^{4}}+\mathcal{O}\left(\alpha^{\prime 4}\right) .
\end{aligned}
$$

For definitions of the $\widehat{\mathcal{I}}_{12 \mid 34}^{(w, 0)}$ to all orders in $\alpha^{\prime}$ via $z$-integrals, see (D.21) and (D.27).

\subsection{The integrated amplitude and the low-energy effective action}

Our main focus of this paper lies on the structure of the $\tau$-integrand $M_{4}(\tau)$ appearing in the four-point gauge amplitude (2.38). However, using the results of [9-11, 23, 26, 76, 77] on the integrals of certain combinations of modular graph forms and Eisenstein series, it is possible to perform the integral over $\tau$ analytically up to second order in $\alpha^{\prime}$ both in the single-trace and the double-trace sector. We present the resulting values, keeping in mind that these have to be considered in our normalization (2.38), see also footnote 7 .

${ }^{20} \mathrm{We}$ are using the Ramanujan identities

$$
q \frac{\mathrm{dG}_{2}}{\mathrm{~d} q}=\frac{\mathrm{G}_{2}^{2}-5 \mathrm{G}_{4}}{4 \pi^{2}}, \quad q \frac{\mathrm{dG}_{4}}{\mathrm{~d} q}=\frac{2 \mathrm{G}_{2} \mathrm{G}_{4}-7 \mathrm{G}_{6}}{2 \pi^{2}}, \quad q \frac{\mathrm{dG}_{6}}{\mathrm{~d} q}=\frac{21 \mathrm{G}_{2} \mathrm{G}_{6}-30 \mathrm{G}_{4}^{2}}{14 \pi^{2}} .
$$




\subsubsection{Planar amplitude up to second order in $\alpha^{\prime}$}

Upon collecting all terms of the same structure in Mandelstam invariants up to quadratic order from (4.50) we have to evaluate the integrals appearing in

$$
\begin{aligned}
& \left.\mathcal{M}_{4}\right|_{\operatorname{Tr}\left(t^{a_{1}} t^{a_{2}} t^{\left.a_{3} t^{a_{4}}\right)}\right.} \sim \int_{\mathcal{F}} \frac{\mathrm{d}^{2} \tau}{(\operatorname{Im} \tau)^{2} \eta^{24}}\left(\frac{49}{6} \mathrm{G}_{6}^{2}-\frac{10}{3} \mathrm{G}_{4}^{3}\right) \\
& +s_{13} \int_{\mathcal{F}} \frac{\mathrm{d}^{2} \tau}{(\operatorname{Im} \tau)^{2} \eta^{24}}\left(6 \mathrm{G}_{4}^{3}+3 \mathrm{G}_{4}^{2} \hat{\mathrm{G}}_{2} \frac{\pi \nabla \mathrm{E}_{2}}{(\operatorname{Im} \tau)^{2}}-\frac{21}{2} \mathrm{G}_{4} \mathrm{G}_{6} \frac{\pi \nabla \mathrm{E}_{2}}{(\operatorname{Im} \tau)^{2}}\right) \\
& +\left(s_{13}^{2}-s_{12} s_{23}\right) \int_{\mathcal{F}} \frac{\mathrm{d}^{2} \tau}{(\operatorname{Im} \tau)^{2} \eta^{24}}\left(\frac{49}{3} \mathrm{G}_{4} \mathrm{G}_{6}^{2} \mathrm{E}_{2}-\frac{20}{3} \mathrm{G}_{4}^{3} \mathrm{E}_{2}\right) \\
& +\left(s_{13}^{2}+2 s_{12} s_{23}\right) \int_{\mathcal{F}} \frac{\mathrm{d}^{2} \tau}{(\operatorname{Im} \tau)^{2} \eta^{24}}\left(\frac{2}{3} \mathrm{G}_{4}^{2} \hat{\mathrm{G}}_{2} \frac{\pi \nabla \mathrm{E}_{3}}{(\operatorname{Im} \tau)^{2}}-\frac{7}{3} \mathrm{G}_{4} \mathrm{G}_{6} \frac{\pi \nabla \mathrm{E}_{3}}{(\operatorname{Im} \tau)^{2}}+\frac{1}{6} \mathrm{G}_{4}^{2} \frac{(\pi \nabla)^{2} \mathrm{E}_{3}}{(\operatorname{Im} \tau)^{4}}\right) \\
& +\mathcal{O}\left(\alpha^{\prime 3}\right) \text {. }
\end{aligned}
$$

These integrals can be done using the following observations. The combination of $\mathrm{G}_{4}^{3}$ and $\mathrm{G}_{6}^{2}$ appearing in the first and third line is that of (4.52) leading to $\eta^{24}$, making the first line proportional to the volume of $\mathcal{F}$ that equals $\frac{\pi}{3}$ while the third line is proportional to the integral of $\mathrm{E}_{2}$ over $\mathcal{F}$ that vanishes [9]. Using furthermore the results of $[23,26]$ for the remaining lines, we end up with the integrated planar amplitude

$$
\begin{aligned}
& \left.\mathcal{M}_{4}\right|_{\operatorname{Tr}\left(t^{a_{1}} t^{a_{2}} t^{\left.a_{3} t^{a_{4}}\right)}\right.} \sim-\frac{256 \pi^{13}}{6075}+s_{13} \frac{32 \pi^{13}}{6075}\left(\frac{25}{6}+\gamma_{\mathrm{E}}+\log \pi-2 \frac{\zeta_{4}^{\prime}}{\zeta_{4}}\right) \\
& -\left(s_{13}^{2}+2 s_{12} s_{23}\right) \frac{32 \pi^{13}}{30375}+\mathcal{O}\left(\alpha^{\prime 3}\right) \text {. }
\end{aligned}
$$

The appearance of terms $\log \pi$ and $d \log \zeta$ is due to the method of cutting off the fundamental domain $\mathcal{F}$ as in [26]. Note that these terms as well as the Euler-Mascheroni constant $\gamma_{\mathrm{E}}$ cancel at the second order in $\alpha^{\prime}$, a feature that we shall discuss in more detail in section 4.5.3.

From the point of view of the low-energy effective action, the terms above correspond to single-trace higher-derivative corrections of the schematic form $\operatorname{Tr}\left(F^{4}\right), \operatorname{Tr}\left(D^{2} F^{4}\right)$ and $\operatorname{Tr}\left(D^{4} F^{4}\right)$, respectively. The lowest-order term in the one-loop scattering amplitude was already analyzed in $[23,52,53,78,79]$. The structure of higher-derivative invariants in super Yang-Mills theory was studied for example in [80-82] and the three operators above are of $1 / 2-, 1 / 4$ - and non-BPS type, respectively. General references on the effective action of heterotic string theories include [24, 26, 27, 83-89].

\subsubsection{Non-planar amplitude up to second order in $\alpha^{\prime}$}

The integrated contribution from the double-trace sector can be determined by similar methods by starting from (4.54). The $\tau$-integral to be performed to quadratic order in 
Mandelstam invariants is

$$
\begin{aligned}
& \left.\mathcal{M}_{4}\right|_{\operatorname{Tr}\left(t^{a_{1}} t^{a_{2}}\right) \operatorname{Tr}\left(t^{a_{3}} t^{a_{4}}\right)} \sim \int_{\mathcal{F}} \frac{\mathrm{d}^{2} \tau}{(\operatorname{Im} \tau)^{2} \eta^{24}}\left(\mathrm{G}_{4}^{2} \hat{\mathrm{G}}_{2}^{2}-7 \mathrm{G}_{4} \mathrm{G}_{6} \hat{\mathrm{G}}_{2}+\frac{5}{3} \mathrm{G}_{4}^{3}+\frac{49}{6} \mathrm{G}_{6}^{2}\right) \\
& +s_{12} \int_{\mathcal{F}} \frac{\mathrm{d}^{2} \tau}{(\operatorname{Im} \tau)^{2} \eta^{24}}\left(7 \mathrm{G}_{4} \mathrm{G}_{6} \hat{\mathrm{G}}_{2}-2 \mathrm{G}_{4}^{2} \hat{\mathrm{G}}_{2}^{2}\right) \\
& +s_{12}^{2} \int_{\mathcal{F}} \frac{\mathrm{d}^{2} \tau}{(\operatorname{Im} \tau)^{2} \eta^{24}}\left(\mathrm{G}_{4}^{3}+\hat{\mathrm{G}}_{2}^{2} \mathrm{G}_{4}^{2}\left(3+2 \mathrm{E}_{2}\right)-\frac{2 \hat{\mathrm{G}}_{2} \mathrm{G}_{4}^{2} \pi \nabla \mathrm{E}_{2}}{(\mathrm{Im} \tau)^{2}}-7 \mathrm{G}_{4} \mathrm{G}_{6} \hat{\mathrm{G}}_{2}\left(1+2 \mathrm{E}_{2}\right)\right. \\
& \left.+7 \mathrm{G}_{4} \mathrm{G}_{6} \frac{\pi \nabla \mathrm{E}_{2}}{(\operatorname{Im} \tau)^{2}}+\frac{10}{3} \mathrm{G}_{4}^{3} \mathrm{E}_{2}+\frac{49}{3} \mathrm{G}_{6}^{2} \mathrm{E}_{2}\right) \\
& +s_{13} s_{23} \int_{\mathcal{F}} \frac{\mathrm{d}^{2} \tau}{(\operatorname{Im} \tau)^{2} \eta^{24}}\left(-2 \mathrm{G}_{4}^{3}-2 \mathrm{G}_{4}^{2} \hat{\mathrm{G}}_{2}^{2} \mathrm{E}_{2}-2 \frac{\mathrm{G}_{4}^{2} \hat{\mathrm{G}}_{2} \pi \nabla \mathrm{E}_{2}}{(\operatorname{Im} \tau)^{2}}+14 \mathrm{G}_{4} \mathrm{G}_{6} \hat{\mathrm{G}}_{2} \mathrm{E}_{2}\right. \\
& \left.+7 \frac{\mathrm{G}_{4} \mathrm{G}_{6} \pi \nabla \mathrm{E}_{2}}{(\mathrm{Im} \tau)^{2}}-\frac{10}{3} \mathrm{G}_{4}^{3} \mathrm{E}_{2}-\frac{49}{3} \mathrm{G}_{6}^{2} \mathrm{E}_{2}\right)+\mathcal{O}\left(\alpha^{\prime 3}\right) .
\end{aligned}
$$

These integrals can again be performed using the results of $[11,23,26]$, and we obtain the integrated double-trace amplitude to second order in $\alpha^{\prime}$ as

$$
\begin{aligned}
\left.\mathcal{M}_{4}\right|_{\operatorname{Tr}\left(t^{a_{1}} t^{a_{2}}\right) \operatorname{Tr}\left(t^{a_{3}} t^{a_{4}}\right)} \sim & -\frac{128 \pi^{13}}{6075} s_{12}+s_{12}^{2} \frac{64 \pi^{13}}{3645}\left(-\frac{91}{30}+\gamma_{\mathrm{E}}+\log \pi-2 \frac{\zeta_{4}^{\prime}}{\zeta_{4}}\right) \\
& -s_{13} s_{23} \frac{256 \pi^{13}}{18225}\left(-\frac{11}{6}+\gamma_{\mathrm{E}}+\log \pi-2 \frac{\zeta_{4}^{\prime}}{\zeta_{4}}\right)+\mathcal{O}\left(\alpha^{\prime 3}\right) .
\end{aligned}
$$

We note that there is no lowest-order term in the non-planar sector. As pointed out in [24], this is in agreement with the duality between the heterotic string and the type-I string [90], where $\left(\operatorname{Tr}\left(F^{2}\right)\right)^{2}$ is absent at tree level. The first non-trivial correction term for doubletrace operators is then $\left(\operatorname{Tr}\left(D F^{2}\right)\right)^{2}$, and the eight-derivative order admits two independent kinematic structures.

\subsubsection{Consistency with tree-level amplitudes}

Given the expressions (4.57) and (4.59) for the integrals over $\tau$, the appearance of $\gamma_{\mathrm{E}}+$ $\log \pi-2 \frac{\zeta_{4}^{\prime}}{\zeta_{4}}$ signals an interplay with the non-analytic momentum dependence of the respective amplitude at the same $\alpha^{\prime}$-order, cf. $[10,11]$. The non-analytic part of the four-point gauge amplitude can be inferred to comprise factors of $\log s_{i j}$

- in the planar sector at the order of $\alpha^{\prime}$ but not at the orders of $\alpha^{\prime 0}$ or $\alpha^{\prime 2}$, see (4.57)

- in the non-planar sector at the order of $\alpha^{2}$ but not at the orders of $\alpha^{\prime 0}$ or $\alpha^{1}$, see $(4.59)$

These patterns in the discontinuity of the one-loop amplitude are consistent with the $\alpha^{\prime}$ expansion of the respective tree amplitudes of the heterotic string [83]

$$
\begin{gathered}
\left.\mathcal{M}_{4}^{\text {tree }}\right|_{\operatorname{Tr}\left(t^{a_{1}} t^{a_{2}} t^{a_{3}} t^{a_{4}}\right)} \sim 1+2 \zeta_{3} s_{12} s_{13} s_{23}+\mathcal{O}\left(\alpha^{\prime{ }^{5}}\right) \\
\left.\mathcal{M}_{4}^{\text {tree }}\right|_{\operatorname{Tr}\left(t^{a_{1}} t^{a_{2}}\right) \operatorname{Tr}\left(t^{a_{3}} t^{a_{4}}\right)} \sim s_{23}-s_{12} s_{23}+s_{12}^{2} s_{23}+\mathcal{O}\left(\alpha^{\prime 4}\right),
\end{gathered}
$$


where a polarization-dependent factor $A_{\mathrm{SYM}}^{\text {tree }}(1,2,3,4)$ has been subsumed in the $\sim$. Unitarity relates the $\left(s_{i j}\right)^{w}$-order of (4.60) to the non-analytic terms in the one-loop amplitudes that are signaled by the $\left(s_{i j}\right)^{w+1}$-order in (4.57) and (4.59). In particular, the absence of a subleading order $\alpha^{\prime 1}$ in the planar sector of $\mathcal{M}_{4}^{\text {tree }}$ ties in with the absence of $\gamma_{\mathrm{E}}+\log \pi-2 \frac{\zeta_{4}^{\prime}}{\zeta_{4}}$ at the $\alpha^{\prime 2}$-order of (4.57). This is analogous to the discontinuity structure of the massless type-II amplitude [10, 11], where unitarity relates the $\alpha^{\prime w+1}$-order beyond the one-loop low-energy limit to the $\alpha^{\prime w}$-order of the tree amplitude beyond its supergravity limit.

\subsection{Modular graph forms in the massless $n$-point function}

Although the main focus of this work is on the four-point amplitude involving gauge bosons, we shall now explain how the above techniques can be extended to higher multiplicity and to external gravitons. As we will see, the integration over the punctures in $n$-point one-loop amplitudes of the heterotic string involving any combination of gauge bosons and gravitons boils down to (heterotic) modular graph forms - at any order in the $\alpha^{\prime}$-expansion.

\subsection{1 $n$ external gauge bosons}

For the $n$-point generalization of the amplitude (2.10) among four gauge bosons, the structure of the correlation functions in the integrand is well known. The supersymmetric chiral halves of the vertex operators (2.12) exclusively contribute (complex conjugates of) $f_{i j}^{(w)}$ and holomorphic Eisenstein series $\mathrm{G}_{k}$ to the $n$-point correlators [6]. This has been manifested in this reference by expressing RNS spin sums ${ }^{21}$ of the worldsheet fermions in terms of the $V_{w}$ functions (2.6) and $\mathrm{G}_{k}$, also see [93] for analogous results with two external gauginos and $[24,94]$ for earlier work on the spin sums. The contributions from the worldsheet bosons $\partial_{\bar{z}} X(z, \bar{z})$ are even simpler, they can be straightforwardly integrated out using Wick contractions that yield $\overline{f_{i j}^{(1)}}$ or $\partial_{\bar{z}_{i}} \overline{f_{i j}^{(1)}}$. Likewise, the Kac-Moody currents of $(2.12)$ exclusively contribute $V_{w}$ functions and $\mathrm{G}_{k}$ to the complementary chiral half of the $n$-point correlators [25].

Given these results on the $n$-point integrands, it is important to note that $\mathrm{G}_{k}$ are modular graph forms and that $f_{i j}^{(w)}$ functions admit the same type of lattice-sum representation (4.2) as the Green function (3.1). Then, the Fourier integrals over the $n$ punctures yield momentum-conserving delta functions similar to (4.10), and one is left with the kinds of nested lattice sums that define modular graph forms [15].

Starting from the five-point function, the singularities $\overline{f_{i j}^{(1)}} \sim \frac{1}{\bar{z}_{i j}}+\mathcal{O}(z, \bar{z})$ in the supersymmetric correlators introduce kinematic poles into the integrals over the punctures. Still, the residues of these kinematic poles reduce to lower-multiplicity results and therefore give modular graph forms by an inductive argument.

On these grounds, one-loop scattering of $n$ gauge bosons in the heterotic string boil down to modular graph forms at each order in the $\alpha^{\prime}$-expansion after integrating over the punctures at fixed $\tau$.

\footnotetext{
${ }^{21}$ Also see [91, 92] for recent examples of $f^{(w)}$ functions in manifestly supersymmetric higher-point amplitudes in the pure-spinor formalism.
} 


\subsubsection{Adjoining external gravitons}

The vertex operators of gravitons and gauge bosons in heterotic string theories have the same supersymmetric chiral half. That is why for mixed $n$-point amplitudes involving external gauge bosons and gravitons, the supersymmetric half of the correlator is identical to that of $n$ gauge bosons. Only the non-supersymmetric chiral half of the correlators is sensitive to the species of massless states in the external legs since the graviton vertex operator involves the worldsheet boson $\partial_{z} X(z, \bar{z})$ in the place of the Kac-Moody current [51].

These additional worldsheet bosons of the gravitons contribute (sums of products of) $f_{i j}^{(1)}$ and $\partial_{z_{i}} f_{i j}^{(1)}$ due to Wick contractions and decouple from the current correlators of the gauge bosons. Moreover, they admit zero-mode contractions $\partial_{z_{i}} X\left(z_{i}, \bar{z}_{i}\right) \partial_{\bar{z}_{j}} X\left(z_{j}, \bar{z}_{j}\right) \rightarrow$ $\frac{\pi}{\operatorname{Im} \tau}$ between left and right movers, known from type-II amplitudes [95-98]. These kinds of cross-contractions are specific to amplitudes involving gravitons, and the resulting factors of $\frac{\pi}{\operatorname{Im} \tau}$ have the same modular weight $(1,1)$ as the contributions $f_{i j}^{(1)} \overline{f_{k l}^{(1)}}$ due to separate Wick contractions of the left and right movers.

Hence, the additional contributions due to $\partial_{z} X(z, \bar{z})$ in the graviton vertex operator boil down to $f_{i j}^{(1)}, \partial_{z_{i}} f_{i j}^{(1)}$ or $\frac{\pi}{\operatorname{Im} \tau}$. All of these factors line up with the above statements on the correlators of the supersymmetric chiral half and the currents: the $n$-point correlators for mixed graviton- and gauge-boson amplitudes in heterotic string theories exclusively depend on the punctures via lattice sums

$$
\sum_{p \neq 0} \frac{e^{2 \pi i\left\langle p, z_{i j}\right\rangle}}{p^{m_{i j}} \bar{p}^{n_{i j}}}, \quad m_{i j}, n_{i j} \geq-1
$$

that may be accompanied by powers of $\frac{\pi}{\operatorname{Im} \tau}$ and yield simple Fourier integrals w.r.t. $z_{2}, \ldots, z_{n}$. Each term in the $\alpha^{\prime}$-expansion of the Koba-Nielsen factor is bound to yield modular graph forms upon integrating over the $z_{j}$. By the arguments given for $n$ gauge bosons, the kinematic poles do not alter this result.

Note that cases with $m_{i j}=n_{i j}=-1$ in (4.61) are due to the spurious factors of $\partial_{z_{i}} f_{i j}^{(1)}$ and $\partial_{\bar{z}_{i}} \overline{f_{i j}^{(1)}}$ in the left- and right-moving contributions to the correlators. One can always remove any appearance of $\partial_{z_{i}} f_{i j}^{(1)}$ and $\partial_{\bar{z}_{i}} f_{i j}^{(1)}$ via integration by parts and thereby improve the bound in (4.61) towards $m_{i j}, n_{i j} \geq 0$.

The $\alpha^{\prime}$-expansion of four-point amplitudes involving gravitons has been studied beyond the leading order in $[26,27]$. Moreover, selected terms in the five- and six-point gauge amplitudes that are relevant to $\operatorname{Tr}\left(F^{5}\right)$ and $\operatorname{Tr}\left(F^{6}\right)$ interactions have been studied in [24]. The comparison of $(n \geq 5)$-point $\alpha^{\prime}$-expansions with open-string results is left for the future [99].

\section{$5 \quad$ Heterotic strings versus open superstrings}

In this section, we point out new relations between open-superstring amplitudes and the integral $\mathcal{I}_{1234}^{(2,0)}$ over the torus punctures in the planar sector of the heterotic-string amplitude, cf. (2.42). The observations of this section can be viewed as generalizing the genus-zero result that closed-string amplitudes are single-valued open-string amplitudes [3, 28-32]. That 
is why we motivate the genus-one discussion by a brief review of the tree-level connection between moduli-space integrals in open- and closed-string amplitudes.

\subsection{Review of the single-valued map in tree amplitudes}

The single-valued map sv : $\zeta_{n_{1}, n_{2}, \ldots, n_{r}} \rightarrow \zeta_{n_{1}, n_{2}, \ldots, n_{r}}^{\mathrm{sv}}$ of MZVs [33, 34],

$$
\begin{aligned}
\zeta_{2 k}^{\mathrm{sv}} & =0, & \zeta_{2 k+1}^{\mathrm{sv}} & =2 \zeta_{2 k+1}, \quad k \in \mathbb{N} \\
\zeta_{3,5}^{\mathrm{sv}} & =-10 \zeta_{3} \zeta_{5}, & \zeta_{3,5,3}^{\mathrm{sv}} & =2 \zeta_{3,5,3}-2 \zeta_{3} \zeta_{3,5}-10 \zeta_{3}^{2} \zeta_{5},
\end{aligned}
$$

relates moduli-space integrals over punctured spheres to single-valued disk integrals at the level of their low-energy expansion. Open-string tree amplitudes boil down to disk integrals that can be characterized by an integration domain for the punctures on the boundary

$$
D(1,2, \ldots, n):=\left\{\left(z_{1}, z_{2}, \ldots, z_{n}\right) \in \mathbb{R}^{n}:-\infty<z_{1}<z_{2}<\ldots<z_{n}<\infty\right\}
$$

and a cyclic Parke-Taylor factor $\left(z_{12} z_{23} \ldots z_{n 1}\right)^{-1}$ in the integrand [40, 41, 74, 75],

$$
Z_{\text {tree }}(\rho(1,2, \ldots, n) \mid 1,2, \ldots, n):=\int_{D(\rho(1,2, \ldots, n))} \frac{\mathrm{d} z_{1} \mathrm{~d} z_{2} \ldots \mathrm{d} z_{n}}{\operatorname{vol~SL} L_{2}(\mathbb{R})} \frac{\prod_{i<j}^{n}\left|z_{i j}\right|^{-s_{i j}}}{z_{12} z_{23} \ldots z_{n-1, n} z_{n 1}} .
$$

The ordering of the punctures on the boundary does not need to coincide with that of the contractions giving the Parke-Taylor factors and this is included in the formula above by the permutation $\rho \in S_{n}$.

The sphere integrals arising for the closed string do not admit a similar notion of cyclic ordering of the punctures but instead involve Parke-Taylor integrands in both the holomorphic and the antiholomorphic variables,

$$
J_{\text {tree }}(\rho(1,2, \ldots, n) \mid 1,2, \ldots, n):=\int_{\mathbb{C}^{n}} \frac{\mathrm{d}^{2} z_{1} \mathrm{~d}^{2} z_{2} \ldots \mathrm{d}^{2} z_{n}}{\pi^{n-3} \operatorname{vol~SL}(\mathbb{C})} \frac{\prod_{i<j}^{n}\left|z_{i j}\right|^{-2 s_{i j}}}{z_{12} z_{23} \ldots z_{n 1} \rho\left(\bar{z}_{12} \bar{z}_{23} \ldots \bar{z}_{n 1}\right)},
$$

where the permutation $\rho \in S_{n}$ is understood to act as $\rho\left(\bar{z}_{12} \ldots\right)=\left(\bar{z}_{\rho(1) \rho(2)} \ldots\right)$. The inverse volumes of $\mathrm{SL}_{2}$ instruct to fix any three punctures to $(0,1, \infty)$ with the standard Jacobian $[100,101]$, and the numerator factors $\left|z_{i j}\right|^{-s_{i j}}$ and $\left|z_{i j}\right|^{-2 s_{i j}}$ stem from the genuszero analogues of the plane-wave correlator (2.14).

For any choice of $\rho$, the low-energy expansions of (5.3) and (5.4) are related by the single-valued map (5.1) [3, 28-32],

$$
J_{\text {tree }}(\rho(1,2, \ldots, n) \mid 1,2, \ldots, n)=\operatorname{sv} Z_{\text {tree }}(\rho(1,2, \ldots, n) \mid 1,2, \ldots, n) .
$$

Hence, the cyclic ordering of the integration domain (5.2) on the disk boundary is passed to the antiholomorphic Parke-Taylor factor $\rho\left(\bar{z}_{12} \bar{z}_{23} \ldots \bar{z}_{n 1}\right)^{-1}$ under the single-valued map, following a Betti-deRham duality ${ }^{22}[36,37]$.

\footnotetext{
${ }^{22}$ In the context of graphical hyperlogarithms in [37], inequalities $z_{i}<z_{j}$ and factors of $\left(\bar{z}_{i}-\bar{z}_{j}\right)^{-1}$ are represented by (directed) Betti edges and deRham edges, respectively. By exchanging the roles of these edges, one converts the Parke-Taylor factor $\left(\bar{z}_{12} \bar{z}_{23} \ldots \bar{z}_{n 1}\right)^{-1}$ into a disk ordering $-\infty<z_{1}<z_{2}<\ldots<$ $z_{n}<\infty$.
} 
The single-valued relation (5.5) at tree level allows to extract the complete closedstring low-energy expansions $J_{\text {tree }}$ from the open-string input $Z_{\text {tree }}$ (see e.g. $[4,102]$ for recent methods for open-string $\alpha^{\prime}$-expansions at $n$ points). In particular, the expressions for single-valued MZVs in (5.1) expose the absence of certain MZVs (including $\zeta_{2 k}, \zeta_{3,5}$, also see [103]) in closed-string amplitudes which is obscured in the Kawai-Lewellen-Tye relations [104]. Obtaining a loop-level analogue of the single-valued map (5.5) would similarly allow extracting the closed-string one-loop amplitudes from open-string calculations.

A first hint for the existence of such loop-level relations has been given in [8], where modular graph functions were identified as special values of infinite sums of single-valued multiple polylogarithms. The infinite sums in the reference are proposed to be single-valued analogues of elliptic multiple polylogarithms. Moreover, an explicit connection between open- and closed-string data at genus one has been found in [22], relating symmetrized integrals over open-string punctures ${ }^{23}$ to the permutation-invariant integral $\mathcal{I}^{(0,0)}$ in $(2.45)$. The proposal for an elliptic single-valued map "esv" [22] and the open-string setup will be reviewed below, and we will identify the closed-string counterpart to more general fourpoint open-string integrals.

\subsection{Open-superstring integrals at genus one}

In the same way as the one-loop four-point amplitude of type-II superstrings boils down to a single integral $\mathcal{I}^{(0,0)}$, the single-trace sector of the corresponding open-string amplitude is governed by [55]

$$
I_{1234}^{\text {open }}\left(s_{i j}, \tau\right):=\int_{0 \leq z_{2} \leq z_{3} \leq z_{4} \leq 1} \mathrm{~d} z_{2} \mathrm{~d} z_{3} \mathrm{~d} z_{4} \exp \left(\sum_{i<j}^{4} s_{i j} G_{i j}^{\text {open }}(\tau)\right) .
$$

The integration domain for the punctures $z_{2}, z_{3}, z_{4}$ corresponds to a particular parametrization of a cylinder worldsheet, where the unit interval $(0,1)$ represents one of the boundaries. We have again fixed $z_{1}=0$ by translation invariance, and although the open-string amplitude only involves $\tau=i t, t \in \mathbb{R}_{+}$, we will study the integral (5.6) for arbitrary $\tau$ in the upper half plane. The open-string counterpart to the closed-string Green function (2.15) can be chosen as

$$
G_{i j}^{\text {open }}(\tau)=-\int_{0}^{z_{i j}} \mathrm{~d} w f^{(1)}(w, \tau),
$$

where all $z_{j}$ and $w$ are taken to be on the real axis, and the regularization of the endpoint divergence is discussed in [6]. Given that the Taylor expansion of (5.6) boils down to iterated integrals over the expansion coefficient $f^{(1)}$ in the Kronecker-Eisenstein series (2.4), each order in $\alpha^{\prime}$ is expressible in terms of elliptic multiple zeta values (eMZVs) [6]. More precisely, the parametrization of the cylinder boundary in (5.6) gives rise to the A-cycle

\footnotetext{
${ }^{23}$ The symmetrized integrals of [22] would correspond to scattering of abelian gauge bosons which do not appear in the spectrum of the type-I superstring with gauge group $\mathrm{SO}(32)$. While the physical interpretation of the symmetrized open-string integrals is an open question, the results of this section relate the esv-image of more general four-point open-string integrals compatible with $\mathrm{SO}(32)$ to closed-string quantities.
} 
eMZVs of Enriquez [5]

$$
\omega\left(n_{1}, n_{2}, \ldots, n_{r} \mid \tau\right):=\int_{0 \leq z_{1} \leq z_{2} \leq \ldots \leq z_{r} \leq 1} f^{\left(n_{1}\right)}\left(z_{1}, \tau\right) \mathrm{d} z_{1} f^{\left(n_{2}\right)}\left(z_{2}, \tau\right) \mathrm{d} z_{2} \ldots f^{\left(n_{r}\right)}\left(z_{r}, \tau\right) \mathrm{d} z_{r},
$$

where the number $r$ of arguments $n_{i} \in \mathbb{N}_{0}$ and the sum $n_{1}+n_{2}+\ldots+n_{r}$ are referred to as length and weight, respectively. The differential equations of eMZVs in $\tau$ and their degeneration at the cusp can be used to infer a Fourier expansion $[5,67]$

$$
\omega\left(n_{1}, n_{2}, \ldots, n_{r} \mid \tau\right)=\sum_{k=0}^{\infty} c_{k}\left(n_{1}, n_{2}, \ldots, n_{r}\right) q^{k}
$$

where the $\tau$-independent coefficients $c_{k}\left(n_{1}, \ldots, n_{r}\right)$ are $\mathbb{Q}\left[(2 \pi i)^{-1}\right]$-linear combinations of MZVs. The dependence of the eMZVs on $\tau$ will usually be suppressed for ease of notation. See [105] for a comprehensive reference on eMZVs.

As exemplified by the leading low-energy behavior of (5.6) [6],

$$
\begin{aligned}
I_{1234}^{\text {open }}\left(s_{i j}, \tau\right)= & \frac{1}{6}-2 s_{13} \omega(0,1,0,0)+2\left(s_{12}^{2}+s_{23}^{2}\right) \omega(0,1,1,0,0) \\
& -2 s_{12} s_{23} \omega(0,1,0,1,0)+s_{13}\left(s_{13}^{2}-s_{12} s_{23}\right) \beta_{5}+s_{12} s_{23} s_{13} \beta_{2,3}+\mathcal{O}\left(\alpha^{\prime 4}\right),
\end{aligned}
$$

the order in $\alpha^{\prime}$ is in one-to-one correspondence with the weight of the accompanying eMZVs, i.e. $I_{1234}^{\text {open }}\left(s_{i j}, \tau\right)$ is said to be uniformly transcendental. We have introduced the following shorthands for the coefficients at the third order in $\alpha^{\prime}$,

$$
\begin{aligned}
\beta_{5} & =\frac{4}{3}\left[\omega(0,0,1,0,0,2)+\omega(0,1,1,0,1,0)-\omega(2,0,1,0,0,0)-\zeta_{2} \omega(0,1,0,0)\right] \\
\beta_{2,3} & =\frac{\zeta_{3}}{12}+\frac{8}{3} \zeta_{2} \omega(0,1,0,0)-\frac{5}{18} \omega(0,3,0,0) .
\end{aligned}
$$

In the same way as MZVs descend from multiple polylogarithms, eMZVs are special values of elliptic multiple polylogarithms [49]. Various representations of elliptic multiple polylogarithms have recently found appearance in the evaluation of Feynman integrals, see e.g. [46, 106-117] and references therein.

\subsubsection{Decomposition into symmetry components}

By the properties of the integration cycle and the open-string Green function in (5.6), the open-string integral exhibits the same dihedral symmetries w.r.t. its labels $1,2,3,4$ as the $V_{w}$ functions at even values of $w$,

$$
I_{1234}^{\mathrm{open}}\left(s_{i j}, \tau\right)=I_{2341}^{\mathrm{open}}\left(s_{i j}, \tau\right), \quad I_{4321}^{\mathrm{open}}\left(s_{i j}, \tau\right)=I_{1234}^{\mathrm{open}}\left(s_{i j}, \tau\right),
$$

cf. (2.7). In order to explore further connections with the $V_{w}$ functions, we decompose the integral $I_{1234}^{\text {open }}=\frac{1}{6} Z^{(0)}+Z_{1234}^{(2)}$ into components with different symmetry properties in $1,2,3,4$,

$$
\begin{aligned}
Z^{(0)}\left(s_{i j}, \tau\right) & :=\sum_{\sigma \in S_{3}} I_{1 \sigma(234)}^{\mathrm{open}}\left(s_{i j}, \tau\right) \\
Z_{1234}^{(2)}\left(s_{i j}, \tau\right) & :=\frac{1}{3}\left[2 I_{1234}^{\mathrm{open}}\left(s_{i j}, \tau\right)-I_{1342}^{\mathrm{open}}\left(s_{i j}, \tau\right)-I_{1423}^{\mathrm{open}}\left(s_{i j}, \tau\right)\right] .
\end{aligned}
$$


While the permutation symmetric component $Z^{(0)}$ of the open-string integral has been studied in [22], we will here investigate the $\alpha^{\prime}$-expansion of the second component $Z_{\ldots}^{(2)}$ subject to

$$
Z_{1234}^{(2)}\left(s_{i j}, \tau\right)+Z_{1342}^{(2)}\left(s_{i j}, \tau\right)+Z_{1423}^{(2)}\left(s_{i j}, \tau\right)=0
$$

The symmetry properties of the $Z^{(0)}$ and $Z_{\ldots}^{(2)}$ tie in with those of $V_{0}(1,2,3,4)=1$ and $V_{2}(1,2,3,4)$, respectively, see (4.29).

By inserting the $\alpha^{\prime}$-expansion (5.10) along with momentum conservation $s_{12}+s_{13}+$ $s_{23}=0$ into (5.14), we arrive at the following representation in terms of eMZVs ${ }^{24}$

$$
\begin{aligned}
Z^{(0)}\left(s_{i j}, \tau\right)= & 1+\left(s_{13}^{2}-s_{12} s_{23}\right)\left(2 \omega(0,0,2)+\frac{5 \zeta_{2}}{3}\right)+6 s_{12} s_{23} s_{13} \beta_{2,3}+\mathcal{O}\left(\alpha^{\prime 4}\right) \\
Z_{1234}^{(2)}\left(s_{i j}, \tau\right)= & -2 s_{13} \omega(0,1,0,0)-\frac{2}{3}\left(s_{13}^{2}+2 s_{12} s_{23}\right)[\omega(0,1,0,1,0)+\omega(0,1,1,0,0)] \\
& +s_{13}\left(s_{13}^{2}-s_{12} s_{23}\right) \beta_{5}+\mathcal{O}\left(\alpha^{\prime 4}\right)
\end{aligned}
$$

Note that the coefficient $\beta_{2,3}$ in (5.10) drops out from the definition of $Z_{1234}^{(2)}$ in (5.14), and we are only left with a specific linear combination of $\omega(0,1,0,1,0)$ and $\omega(0,1,1,0,0)$ at order $\alpha^{\prime 2}$.

\subsubsection{Modular transformation}

A connection between the symmetrized open-string integral $Z^{(0)}$ in (5.14) and closed-string integrals [22] is based on the modular $S$ transformation $\tau \rightarrow-\frac{1}{\tau}$ of the contributing eMZVs. Otherwise, the $q$-series representation (5.9) of the A-cycle eMZVs in (5.16) and (5.17) would not exhibit any open-string analogue of the expansion (3.44) of modular graph forms around the cusp, more specifically of the Laurent polynomials $c_{m, n}^{\Gamma}(y)$ in $y=\pi \operatorname{Im} \tau$.

In order to determine the modular $S$ transformation of the $Z_{\ldots}^{(w)}$ integrals in (5.16) and (5.17), we express the A-cycle eMZVs in terms of iterated Eisenstein integrals $(3.38)[67,118]$

$$
\begin{aligned}
& Z^{(0)}\left(s_{i j}, \tau\right)= 1+\left(s_{12} s_{23}-s_{13}^{2}\right)\left[12 \mathcal{E}_{0}(4,0)-\zeta_{2}\right] \\
&-s_{12} s_{23} s_{13}\left[12 \mathcal{E}_{0}(4,0,0)+300 \mathcal{E}_{0}(6,0,0)-\frac{5 \zeta_{3}}{2}\right]+\mathcal{O}\left(\alpha^{\prime 4}\right) \\
& Z_{1234}^{(2)}\left(s_{i j}, \tau\right)= \frac{3 s_{13}}{2 \pi^{2}}\left[6 \mathcal{E}_{0}(4,0,0)-\zeta_{3}\right]+\frac{s_{13}^{2}+2 s_{12} s_{23}}{2 \pi^{2}}\left[120 \mathcal{E}_{0}(6,0,0,0)-\zeta_{4}\right] \\
&+ \frac{s_{13}\left(s_{13}^{2}-s_{12} s_{23}\right)}{2 \pi^{2}}\left[1296 \mathcal{E}_{0}(4,4,0,0,0)+432 \mathcal{E}_{0}(4,0,4,0,0)+\frac{6}{5} \mathcal{E}_{0}(4,0,0,0,0)\right. \\
&\left.\quad+4032 \mathcal{E}_{0}(8,0,0,0,0)-216 \mathcal{E}_{0}(4,0) \mathcal{E}_{0}(4,0,0)+36 \zeta_{3} \mathcal{E}_{0}(4,0)-5 \zeta_{5}\right]+\mathcal{O}\left(\alpha^{\prime 4}\right)
\end{aligned}
$$

${ }^{24}$ We have used the following relations among eMZVs in simplifying (5.16) [67]

$$
\omega(0,1,1,0,0)=\frac{\zeta_{2}}{12}+\omega(0,0,0,0,2), \quad \omega(0,1,0,1,0)=\frac{\zeta_{2}}{12}+\frac{1}{2} \omega(0,0,2)-4 \omega(0,0,0,0,2) .
$$


The modular properties of the holomorphic Eisenstein series give rise to $S$-transformations such as $[22,69]$

$$
\begin{aligned}
\mathcal{E}_{0}\left(4,0 ;-\frac{1}{\tau}\right)= & \frac{T^{2}}{1080}+\frac{\pi^{2}}{216}-\frac{i \zeta_{3}}{6 T}-\frac{\pi^{4}}{360 T^{2}}+\mathcal{E}_{0}(4,0 ; \tau)+\frac{i}{T} \mathcal{E}_{0}(4,0,0 ; \tau) \\
\mathcal{E}_{0}\left(4,0,0 ;-\frac{1}{\tau}\right)= & \frac{i \pi^{2} T}{540}+\frac{\zeta_{3}}{6}-\frac{i \pi^{4}}{108 T}-\frac{\pi^{2} \zeta_{3}}{6 T^{2}}+\frac{i \pi^{6}}{540 T^{3}}+\frac{\pi^{2}}{T^{2}} \mathcal{E}_{0}(4,0,0 ; \tau) \\
\mathcal{E}_{0}\left(6,0,0 ;-\frac{1}{\tau}\right)= & -\frac{i T^{3}}{226800}+\frac{i \pi^{4}}{21600 T}+\frac{\zeta_{5}}{40 T^{2}}-\frac{i \pi^{6}}{22680 T^{3}} \\
& +\mathcal{E}_{0}(6,0,0 ; \tau)+\frac{3 i}{T} \mathcal{E}_{0}(6,0,0,0 ; \tau)-\frac{3}{T^{2}} \mathcal{E}_{0}(6,0,0,0,0 ; \tau) \\
\mathcal{E}_{0}\left(6,0,0,0 ;-\frac{1}{\tau}\right)= & \frac{\pi^{2} T^{2}}{226800}+\frac{\pi^{4}}{64800}+\frac{\pi^{6}}{21600 T^{2}}-\frac{i \pi^{2} \zeta_{5}}{60 T^{3}}-\frac{\pi^{8}}{45360 T^{4}} \\
& +\frac{\pi^{2}}{T^{2}} \mathcal{E}_{0}(6,0,0,0 ; \tau)+\frac{2 i \pi^{2}}{T^{3}} \mathcal{E}_{0}(6,0,0,0,0 ; \tau)
\end{aligned}
$$

and similar to (3.42), we have absorbed powers of $\pi$ into $^{25}$

$$
T:=\pi \tau \text {. }
$$

The remaining modular transformations relevant to (5.19) are displayed in appendix E.1. These expressions for $\mathcal{E}_{0}\left(k_{1}, \ldots ;-\frac{1}{\tau}\right)$ yield the following modular $\tau \rightarrow-\frac{1}{\tau}$ image of (5.18) [22]

$$
\begin{gathered}
Z^{(0)}\left(s_{i j},-\frac{1}{\tau}\right)=1+\left(s_{13}^{2}-s_{12} s_{23}\right)\left(-\frac{T^{2}}{90}+\frac{\pi^{2}}{9}+\frac{2 i \zeta_{3}}{T}+\frac{\pi^{4}}{30 T^{2}}-12 \mathcal{E}_{0}(4,0)-\frac{12 i}{T} \mathcal{E}_{0}(4,0,0)\right) \\
+s_{12} s_{23} s_{13}\left(\frac{i T^{3}}{756}-\frac{i \pi^{2} T}{45}+\frac{\zeta_{3}}{2}+\frac{7 i \pi^{4}}{72 T}+\frac{2 \pi^{2} \zeta_{3}}{T^{2}}-\frac{15 \zeta_{5}}{2 T^{2}}-\frac{17 i \pi^{6}}{1890 T^{3}}-\frac{12 \pi^{2}}{T^{2}} \mathcal{E}_{0}(4,0,0)\right. \\
\left.-300 \mathcal{E}_{0}(6,0,0)-\frac{900 i}{T} \mathcal{E}_{0}(6,0,0,0)+\frac{900}{T^{2}} \mathcal{E}_{0}(6,0,0,0,0)\right)+\mathcal{O}\left(\alpha^{\prime 4}\right)
\end{gathered}
$$

and the following result for (5.19)

$$
\begin{aligned}
& Z_{1234}^{(2)}\left(s_{i j},-\frac{1}{\tau}\right)=s_{13}\left(\frac{i T}{60}-\frac{3 \zeta_{3}}{2 T^{2}}-\frac{i \pi^{2}}{12 T}+\frac{i \pi^{4}}{60 T^{3}}+\frac{9}{T^{2}} \mathcal{E}_{0}(4,0,0)\right) \\
& \quad+\left(s_{13}^{2}+2 s_{12} s_{23}\right)\left(\frac{T^{2}}{3780}-\frac{i \zeta_{5}}{T^{3}}-\frac{\pi^{2}}{216}+\frac{\pi^{4}}{360 T^{2}}-\frac{\pi^{6}}{756 T^{4}}+\frac{60}{T^{2}} \mathcal{E}_{0}(6,0,0,0)+\frac{120 i}{T^{3}} \mathcal{E}_{0}(6,0,0,0,0)\right) \\
& \quad+s_{13}\left(s_{13}^{2}-s_{12} s_{23}\right) \beta_{5}\left(-\frac{1}{\tau}\right)+\mathcal{O}\left(\alpha^{\prime 4}\right)
\end{aligned}
$$

where the modular $\mathrm{S}$ transformation of $\beta_{5}(\tau)$ is given by

$$
\begin{aligned}
\beta_{5}\left(-\frac{1}{\tau}\right)= & -\frac{i T^{3}}{7560}+\frac{i \pi^{2} T}{540}-\frac{\zeta_{3}}{20}-\frac{i \pi^{4}}{120 T}-\frac{5 \zeta_{5}}{2 T^{2}}+\frac{\pi^{2} \zeta_{3}}{12 T^{2}}+\frac{29 i \pi^{6}}{11340 T^{3}}+\frac{\pi^{4} \zeta_{3}}{60 T^{4}}+\frac{3 \zeta_{7}}{T^{4}}-\frac{i \pi^{8}}{1800 T^{5}} \\
& +\left(-\frac{i T}{5}+\frac{i \pi^{2}}{T}+\frac{18 \zeta_{3}}{T^{2}}-\frac{i \pi^{4}}{5 T^{3}}\right) \mathcal{E}_{0}(4,0)+\left(\frac{3}{10}-\frac{\pi^{2}}{2 T^{2}}-\frac{\pi^{4}}{10 T^{4}}\right) \mathcal{E}_{0}(4,0,0) \\
& -\frac{108}{T^{2}} \mathcal{E}_{0}(4,0) \mathcal{E}_{0}(4,0,0)+\frac{216}{T^{2}}\left(\mathcal{E}_{0}(4,0,4,0,0)+3 \mathcal{E}_{0}(4,4,0,0,0)+\frac{\mathcal{E}_{0}(4,0,0,0,0)}{360}\right) \\
& +\frac{2016}{T^{2}} \mathcal{E}_{0}(8,0,0,0,0)+\frac{10080 i}{T^{3}} \mathcal{E}_{0}(8,0,0,0,0,0)-\frac{15120}{T^{4}} \mathcal{E}_{0}(8,0,0,0,0,0,0) .
\end{aligned}
$$

\footnotetext{
${ }^{25}$ Note that this convention for $T$ agrees with the first arXiv version of [22] but not with later versions of the reference, where another factor of $i$ will be absorbed into the definition of $T$.
} 
The $\mathcal{E}_{0}(\ldots)$ on the right-hand sides of $(5.22)$ to $(5.24)$ are understood to be evaluated at argument $\tau$ rather than $-\frac{1}{\tau}$. Note that from the results of [6], any order in the $\alpha^{\prime}$-expansion of (5.22) and (5.23) is expressible in terms of the B-cycle eMZVs of Enriquez [5]. A general discussion of the asymptotic expansion of B-cycle eMZVs around the cusp can be found in $[5,22,70,119]$.

\subsection{A proposal for a single-valued map at genus one}

After modular transformation, the open-string expressions (5.22) to (5.24) resemble the expansion (3.44) of modular graph forms around the cusp: the Laurent polynomials of (5.22) in $T=\pi \tau$ parallel the Laurent polynomials of modular graph forms in $y=\pi \operatorname{Im} \tau$. For instance, with the representations (3.41) of $\mathrm{E}_{k}$ in terms of iterated Eisenstein integrals, the $\alpha^{\prime}$-expansion $(3.9)$ of the closed-string integral $\mathcal{I}^{(0,0)}$ takes the following form,

$$
\begin{aligned}
\mathcal{I}^{(0,0)}\left(s_{i j}, \tau\right)= & 1+2\left(s_{13}^{2}-s_{12} s_{23}\right)\left(\frac{y^{2}}{45}+\frac{\zeta_{3}}{y}-12 \operatorname{Re}\left[\mathcal{E}_{0}(4,0)\right]-\frac{6}{y} \operatorname{Re}\left[\mathcal{E}_{0}(4,0,0)\right]\right) \\
+ & s_{12} s_{23} s_{13}\left(\frac{2 y^{3}}{189}+\zeta_{3}+\frac{15 \zeta_{5}}{4 y^{2}}-600 \operatorname{Re}\left[\mathcal{E}_{0}(6,0,0)\right]\right. \\
& \left.\quad-\frac{900}{y} \operatorname{Re}\left[\mathcal{E}_{0}(6,0,0,0)\right]-\frac{450}{y^{2}} \operatorname{Re}\left[\mathcal{E}_{0}(6,0,0,0,0)\right]\right)+\mathcal{O}\left(\alpha^{\prime 4}\right) .
\end{aligned}
$$

Moreover, the leading low-energy orders $\mathcal{I}^{(0,0)}=1+\mathcal{O}\left(s_{i j}^{2}\right)$ line up with the symmetry component $Z^{(0)}$ of the open-string integral, cf. (5.16), whereas the expansion of $Z_{1234}^{(2)}$ starts at $\mathcal{O}\left(s_{i j}\right)$. That is why the expression (5.25) was compared with the modular S transformation of the symmetrized open-string integral in (5.22) [22]. In fact, the coefficients of the Mandelstam polynomials $s_{13}^{2}-s_{12} s_{23}$ and $s_{12} s_{23} s_{13}$ on the open- and closed-string side were observed to be related via [22]

$$
\text { esv }: \quad T \rightarrow 2 i y, \quad \mathcal{E}_{0}\left(k_{1}, k_{2}, \ldots, k_{r}\right) \rightarrow 2 \operatorname{Re} \mathcal{E}_{0}\left(k_{1}, k_{2}, \ldots, k_{r}\right), \quad \zeta_{n_{1}, \ldots, n_{r}} \rightarrow \zeta_{n_{1}, \ldots, n_{r}}^{\mathrm{sv}}
$$

with $k_{1} \neq 0$. The single-valued MZVs of depth one can be found in (5.1), and the first part $\tau \rightarrow 2 i \operatorname{Im} \tau$ of the esv map in (5.26) does not apply to the exponents in the series representation (3.40) of iterated Eisenstein integrals in $q=e^{2 \pi i \tau}$. The factors of $2 i$ and 2 in (5.26) ensure that the holomorphic derivatives of $\tau$ and $\mathcal{E}_{0}(\ldots ; \tau)$ are preserved under esv, and they were engineered in [22] to obtain

$$
\begin{aligned}
\operatorname{esv} Z^{(0)}\left(s_{i j},-\frac{1}{\tau}\right)= & 1+2\left(s_{13}^{2}-s_{12} s_{23}\right)\left(\frac{y^{2}}{45}+\frac{\zeta_{3}}{y}-12 \operatorname{Re}\left[\mathcal{E}_{0}(4,0)\right]-\frac{6}{y} \operatorname{Re}\left[\mathcal{E}_{0}(4,0,0)\right]\right) \\
+ & +s_{12} s_{23} s_{13}\left(\frac{2 y^{3}}{189}+\zeta_{3}+\frac{15 \zeta_{5}}{4 y^{2}}-600 \operatorname{Re}\left[\mathcal{E}_{0}(6,0,0)\right]\right. \\
& \left.\quad-\frac{900}{y} \operatorname{Re}\left[\mathcal{E}_{0}(6,0,0,0)\right]-\frac{450}{y^{2}} \operatorname{Re}\left[\mathcal{E}_{0}(6,0,0,0,0)\right]\right)+\mathcal{O}\left(\alpha^{\prime 4}\right)
\end{aligned}
$$

which exactly matches (5.25) to the orders shown,

$$
\mathcal{I}^{(0,0)}\left(s_{i j}, \tau\right)=\operatorname{esv} Z^{(0)}\left(s_{i j},-\frac{1}{\tau}\right) .
$$


In fact, this correspondence has been checked to persist up to and including the order of $\alpha^{\prime 6}$ and is conjectural at higher orders [22].

The relation (5.28) between open- and closed-string $\alpha^{\prime}$-expansions at genus one strongly resembles the tree-level relation (5.5) between disk and sphere integrals. Hence, the rules in (5.26) were proposed [22] to implement an elliptic analogue of the single-valued map (5.1) of MZVs.

However, the formulation of the esv rules in (5.28) is in general ill-defined as it is not compatible with the shuffle multiplication of iterated Eisenstein integrals (3.38) [22]. For instance, applying the esv rules to the right-hand side of

$$
\mathcal{E}_{0}(4,0,0)^{2}=2 \mathcal{E}_{0}(4,0,0,4,0,0)+6 \mathcal{E}_{0}(4,0,4,0,0,0)+12 \mathcal{E}_{0}(4,4,0,0,0,0)
$$

yields a different result than the square of esv $\mathcal{E}_{0}(4,0,0)=\mathcal{E}_{0}(4,0,0)+\overline{\mathcal{E}_{0}(4,0,0)}$. The ambiguity in applying esv to (5.29) is proportional to the cross-term $\mathcal{E}_{0}(4,0,0) \overline{\mathcal{E}_{0}(4,0,0)}$ which is of order $\mathcal{O}(q \bar{q})$ by the $q$-expansion (3.40). More generally, terms of the form $q^{n} \bar{q}^{0}$ and $q^{0} \bar{q}^{n}$ with $n \in \mathbb{N}_{0}$ in the output of the esv rules (5.26) are well-defined, i.e. independent of the order of applying shuffle multiplication and esv. We will later on encounter a similar restriction on the powers of $q$ and $\bar{q}$ in the expansion (3.44) of modular graph forms that can be reliably predicted from open-string input.

For the iterated Eisenstein integrals of depth one in (5.22), the convergent $\mathcal{E}_{0}(k, 0, \ldots, 0)$ with $k \geq 4$ cannot be rewritten via shuffle multiplication without introducing divergent examples $\mathcal{E}_{0}(0, \ldots)$. By restricting the esv rules (5.26) to convergent iterated Eisenstein integrals, the ambiguities due to shuffle multiplication are relegated to depth two. The relation (5.28) has been established to the order of $\alpha^{\prime 6}$ (including $\mathcal{E}_{0}$ of depth three) by picking an ad hoc convention for the use of shuffle-multiplication in the open-string input, see section 4.3 .3 of [22] for details.

Note that a reformulation of the esv action on $\mathcal{E}_{0}(\ldots)$ that bypasses the issues with the shuffle multiplication and should make contact with the equivariant Eisenstein integrals of [39] will be discussed in [120].

\subsection{The closed-string integral over $V_{2}(1,2,3,4)$ versus esv $Z_{1234}^{(2)}$}

Given the above significance of the symmetrized open-string integral $Z^{(0)}$, we will next apply the esv rules (5.26) to the symmetry component $Z_{1234}^{(2)}$ in (5.14). Starting from the low-energy expansion (5.23) and (5.24) of its modular S transformation, one arrives at

$$
\begin{aligned}
\text { esv } & Z_{1234}^{(2)}\left(s_{i j},-\frac{1}{\tau}\right)=s_{13}\left(-\frac{y}{30}+\frac{3 \zeta_{3}}{4 y^{2}}-\frac{9}{2 y^{2}} \operatorname{Re}\left[\mathcal{E}_{0}(4,0,0)\right]\right) \\
+ & \left(s_{13}^{2}+2 s_{12} s_{23}\right)\left(-\frac{y^{2}}{945}+\frac{\zeta_{5}}{4 y^{3}}-\frac{30}{y^{2}} \operatorname{Re}\left[\mathcal{E}_{0}(6,0,0,0)\right]-\frac{30}{y^{3}} \operatorname{Re}\left[\mathcal{E}_{0}(6,0,0,0,0)\right]\right) \\
+ & s_{13}\left(s_{13}^{2}-s_{12} s_{23}\right)\left(-\frac{y^{3}}{945}-\frac{\zeta_{3}}{10}+\frac{5 \zeta_{5}}{4 y^{2}}+\frac{3 \zeta_{7}}{8 y^{4}}+\left[\frac{4 y}{5}-\frac{18 \zeta_{3}}{y^{2}}\right] \operatorname{Re}\left[\mathcal{E}_{0}(4,0)\right]+\frac{3}{5} \operatorname{Re}\left[\mathcal{E}_{0}(4,0,0)\right]\right. \\
& -\frac{108}{y^{2}} \operatorname{Re}\left[\mathcal{E}_{0}(4,0,4,0,0)+3 \mathcal{E}_{0}(4,4,0,0,0)+\frac{1}{360} \mathcal{E}_{0}(4,0,0,0,0)\right] \\
& +\frac{108}{y^{2}} \operatorname{Re}\left[\mathcal{E}_{0}(4,0)\right] \operatorname{Re}\left[\mathcal{E}_{0}(4,0,0)\right]-\frac{1008}{y^{2}} \operatorname{Re}\left[\mathcal{E}_{0}(8,0,0,0,0)\right] \\
& \left.-\frac{2520}{y^{3}} \operatorname{Re}\left[\mathcal{E}_{0}(8,0,0,0,0,0)\right]-\frac{1890}{y^{4}} \operatorname{Re}\left[\mathcal{E}_{0}(8,0,0,0,0,0,0)\right]\right)+\mathcal{O}\left(\alpha^{\prime 4}\right)
\end{aligned}
$$

which will now be related to the $\alpha^{\prime}$-expansion of closed-string integrals. 


\subsubsection{The closed-string expansion in terms of iterated Eisenstein integrals}

Given that the symmetry properties (5.15) of $Z_{1234}^{(2)}$ have been tailored to match those of $V_{2}(1,2,3,4)$, it is natural to compare $(5.30)$ with the integral $\mathcal{I}_{1234}^{(2,0)}$ from the heterotic string. The low-energy expansion (4.35) of $\mathcal{I}_{1234}^{(2,0)}$ is written in terms of Cauchy-Riemann derivatives of modular graph functions and can therefore be expressed in terms of iterated Eisenstein integrals. More precisely, the representations (3.41) and (3.43) of $\mathrm{E}_{k}$ and $\mathrm{E}_{2,2}$ yield [22]

$$
\begin{aligned}
\pi \nabla \mathrm{E}_{2}= & \frac{2 y^{3}}{45}-\zeta_{3}+24 y^{2} \mathcal{E}_{0}(4)+12 y \mathcal{E}_{0}(4,0)+6 \operatorname{Re}\left[\mathcal{E}_{0}(4,0,0)\right] \\
\pi \nabla \mathrm{E}_{3}= & \frac{2 y^{4}}{315}-\frac{3 \zeta_{5}}{2 y}+240 y^{2} \mathcal{E}_{0}(6,0)+360 y \mathcal{E}_{0}(6,0,0)+180 \mathcal{E}_{0}(6,0,0,0) \\
& +180 \operatorname{Re}\left[\mathcal{E}_{0}(6,0,0,0)\right]+\frac{180 \operatorname{Re}\left[\mathcal{E}_{0}(6,0,0,0,0)\right]}{y} \\
\pi \nabla \mathrm{E}_{4}= & \frac{4 y^{5}}{4725}-\frac{15 \zeta_{7}}{8 y^{2}}+3360 y^{2} \mathcal{E}_{0}\left(8,0^{2}\right)+10080 y \mathcal{E}_{0}\left(8,0^{3}\right)+12600 \mathcal{E}_{0}\left(8,0^{4}\right) \\
& +\frac{6300 \mathcal{E}_{0}\left(8,0^{5}\right)}{y}+5040 \operatorname{Re}\left[\mathcal{E}_{0}\left(8,0^{4}\right)\right]+\frac{12600 \operatorname{Re}\left[\mathcal{E}_{0}\left(8,0^{5}\right)\right]}{y}+\frac{9450 \operatorname{Re}\left[\mathcal{E}_{0}\left(8,0^{6}\right)\right]}{y^{2}} \\
\pi \nabla \mathrm{E}_{2,2}= & -\frac{2 y^{5}}{10125}+\frac{y^{2} \zeta_{3}}{45}-\frac{5 \zeta_{5}}{12}+\frac{\zeta_{3}^{2}}{2 y}+\left(\frac{4 y^{3}}{15}-6 \zeta_{3}\right) \mathcal{E}_{0}(4,0)-\left(\frac{2 y^{2}}{15}+\frac{6 \zeta_{3}}{y}\right) \operatorname{Re}\left[\mathcal{E}_{0}(4,0,0)\right] \\
& +\frac{2 y^{2}}{5} \mathcal{E}_{0}(4,0,0)+36 y \mathcal{E}_{0}(4,0)^{2}+36 \mathcal{E}_{0}(4,0) \operatorname{Re}\left[\mathcal{E}_{0}(4,0,0)\right]+\frac{18 \operatorname{Re}\left[\mathcal{E}_{0}(4,0,0)\right]^{2}}{y} \\
& +144 y^{2} \mathcal{E}_{0}(4,4,0)+72 y\left(\mathcal{E}_{0}(4,4,0,0)+\frac{1}{360} \mathcal{E}_{0}(4,0,0,0)\right) \\
& +36 \operatorname{Re}\left[\mathcal{E}_{0}(4,0,4,0,0)+3 \mathcal{E}_{0}(4,4,0,0,0)+\frac{1}{360} \mathcal{E}_{0}(4,0,0,0,0)\right]
\end{aligned}
$$

which can be used to cast the leading orders of (4.35) into the following form

$$
\begin{aligned}
\mathcal{I}_{1234}^{(2,0)}\left(s_{i j}, \tau\right)= & \pi^{2} s_{13}\left(\frac{2 y}{15}-\frac{3 \zeta_{3}}{y^{2}}+\frac{18}{y^{2}} \operatorname{Re}\left[\mathcal{E}_{0}(4,0,0)\right]+72 \mathcal{E}_{0}(4)+\frac{36}{y} \mathcal{E}_{0}(4,0)\right) \\
+ & \pi^{2}\left(s_{13}^{2}+2 s_{12} s_{23}\right)\left(\frac{4 y^{2}}{945}-\frac{\zeta_{5}}{y^{3}}+\frac{120}{y^{2}} \operatorname{Re}\left[\mathcal{E}_{0}(6,0,0,0)\right]+\frac{120}{y^{3}} \operatorname{Re}\left[\mathcal{E}_{0}(6,0,0,0,0)\right]\right. \\
& \left.+160 \mathcal{E}_{0}(6,0)+\frac{240}{y} \mathcal{E}_{0}(6,0,0)+\frac{120}{y^{2}} \mathcal{E}_{0}(6,0,0,0)\right)+\mathcal{O}\left(\alpha^{\prime 3}\right) .
\end{aligned}
$$

A similar expression for the $\alpha^{\prime 3}$-order is displayed in appendix E.2. There is a notable difference between the terms involving real parts of iterated Eisenstein integrals $\operatorname{Re}\left[\mathcal{E}_{0}\right]$ and the terms without real parts. The real parts $\operatorname{Re}\left[\mathcal{E}_{0}\right]$ and the pure $y$-terms match the esv image of the open-string integral in (5.30) up to a global rescaling of esv $Z_{1234}^{(2)}\left(s_{i j},-\frac{1}{\tau}\right)$. By contrast, the iterated Eisenstein integrals without real part - specifically, the above $72 \mathcal{E}_{0}(4)$ and $\frac{36}{y} \mathcal{E}_{0}(4,0)$ as well as the last line of $(5.32)$ - do not have any open-string counterpart in (5.30). The same mismatch also arises at the third order in $\alpha^{\prime}$, cf. (E.3). 


\subsubsection{The $P_{\text {Re }}$ projection}

We shall now give a more precise description of the commonalities and differences of the expressions (5.30) and (5.32) for esv $Z_{1234}^{(2)}\left(s_{i j},-\frac{1}{\tau}\right)$ and $\mathcal{I}_{1234}^{(2,0)}\left(s_{i j}, \tau\right)$. The contributions to (5.32) which do not have any obvious open-string correspondent will be isolated by defining a formal projection $P_{\mathrm{Re}}$ via

$$
P_{\operatorname{Re}}\left(\overline{\mathcal{E}_{0}\left(k_{1}, \ldots, k_{r}\right)}\right):=2 \operatorname{Re}\left[\mathcal{E}_{0}\left(k_{1}, \ldots, k_{r}\right)\right], \quad P_{\operatorname{Re}}\left(\mathcal{E}_{0}\left(k_{1}, \ldots, k_{r}\right)\right):=0
$$

with $k_{1} \neq 0$ which acts factor-wise on a product. The projection $P_{\text {Re }}$ is designed to only keep the real parts of iterated Eisenstein integrals, i.e. the cases where holomorphic and antiholomorphic terms pair up. Moreover, Laurent polynomials in $y$ and MZVs are taken to be inert

$$
P_{\operatorname{Re}}\left(y^{m} \zeta_{n_{1}, n_{2}, \ldots, n_{r}}\right):=y^{m} \zeta_{n_{1}, n_{2}, \ldots, n_{r}}
$$

Similar to the esv rule (5.26), the action of $P_{\operatorname{Re}}$ on $\overline{\mathcal{E}_{0}\left(k_{1}, \ldots, k_{r}\right)}$ is incompatible with shuffle multiplication and necessitates ad-hoc conventions for the presentation of its input when two or more of the entries $k_{j}$ are non-zero. By the expansions $\mathcal{E}_{0}\left(k_{1}, \ldots\right)=\mathcal{O}(q)$ and $\overline{\mathcal{E}_{0}\left(k_{1}, \ldots\right)}=\mathcal{O}(\bar{q})$ for $k_{1} \neq 0$, the ambiguity in evaluating $P_{\text {Re }}$ has again at least one factor of both $q$ and $\bar{q}$. Based on their representation given in (5.31), the modular graph forms relevant to the expansion (4.35) are mapped to

$$
\begin{aligned}
P_{\operatorname{Re}}\left(\pi \nabla \mathrm{E}_{2}\right)= & \frac{2 y^{3}}{45}-\zeta_{3}+6 \operatorname{Re}\left[\mathcal{E}_{0}(4,0,0)\right] \\
P_{\operatorname{Re}}\left(\pi \nabla \mathrm{E}_{3}\right)= & \frac{2 y^{4}}{315}-\frac{3 \zeta_{5}}{2 y}+180 \operatorname{Re}\left[\mathcal{E}_{0}(6,0,0,0)\right]+\frac{180 \operatorname{Re}\left[\mathcal{E}_{0}(6,0,0,0,0)\right]}{y} \\
P_{\operatorname{Re}}\left(\pi \nabla \mathrm{E}_{4}\right)= & \frac{4 y^{5}}{4725}-\frac{15 \zeta_{7}}{8 y^{2}}+5040 \operatorname{Re}\left[\mathcal{E}_{0}(8,0,0,0,0)\right] \\
& +\frac{12600 \operatorname{Re}\left[\mathcal{E}_{0}(8,0,0,0,0,0)\right]}{y}+\frac{9450 \operatorname{Re}\left[\mathcal{E}_{0}(8,0,0,0,0,0,0)\right]}{y^{2}} \\
P_{\operatorname{Re}}\left(\pi \nabla \mathrm{E}_{2,2}\right)= & -\frac{2 y^{5}}{10125}+\frac{y^{2} \zeta_{3}}{45}-\frac{5 \zeta_{5}}{12}+\frac{\zeta_{3}^{2}}{2 y}-\left(\frac{2 y^{2}}{15}+\frac{6 \zeta_{3}}{y}\right) \operatorname{Re}\left[\mathcal{E}_{0}(4,0,0)\right]+\frac{18 \operatorname{Re}\left[\mathcal{E}_{0}(4,0,0)\right]^{2}}{y} \\
& +36 \operatorname{Re}\left[\mathcal{E}_{0}(4,0,4,0,0)+3 \mathcal{E}_{0}(4,4,0,0,0)+\frac{1}{360} \mathcal{E}_{0}(4,0,0,0,0)\right] .
\end{aligned}
$$

The expression (3.41) for $\mathrm{E}_{2}$ is invariant under the projection (5.33), so it naturally extends to the product

$$
\begin{aligned}
P_{\operatorname{Re}}\left(\mathrm{E}_{2} \pi \nabla \mathrm{E}_{2}\right)= & \frac{2 y^{5}}{2025}+\frac{y^{2} \zeta_{3}}{45}-\frac{\zeta_{3}^{2}}{y}+\left(12 \zeta_{3}-\frac{8 y^{3}}{15}\right) \operatorname{Re}\left[\mathcal{E}_{0}(4,0)\right]+\left(\frac{12 \zeta_{3}}{y}-\frac{2 y^{2}}{15}\right) \operatorname{Re}\left[\mathcal{E}_{0}(4,0,0)\right] \\
& -72 \operatorname{Re}\left[\mathcal{E}_{0}(4,0)\right] \operatorname{Re}\left[\mathcal{E}_{0}(4,0,0)\right]-\frac{36}{y} \operatorname{Re}\left[\mathcal{E}_{0}(4,0,0)\right]^{2}
\end{aligned}
$$




\subsubsection{The relation between $Z_{1234}^{(2)}$ and $\mathcal{I}_{1234}^{(2,0)}$}

When applied to the low-energy expansion (5.32) and (E.3) of $\mathcal{I}^{(2,0)}$, the projection $P_{\mathrm{Re}}$ removes all standalone instances of $\mathcal{E}_{0}$ but preserves the real parts $P_{\operatorname{Re}} \operatorname{Re}\left[\mathcal{E}_{0}\right]=\operatorname{Re}\left[\mathcal{E}_{0}\right]$ :

$$
\begin{aligned}
& P_{\operatorname{Re}}\left(\mathcal{I}_{1234}^{(2,0)}\left(s_{i j}, \tau\right)\right)=\pi^{2} s_{13}\left(\frac{2 y}{15}-\frac{3 \zeta_{3}}{y^{2}}+\frac{18}{y^{2}} \operatorname{Re}\left[\mathcal{E}_{0}(4,0,0)\right]\right) \\
&+\pi^{2}\left(s_{13}^{2}+2 s_{12} s_{23}\right)\left(\frac{4 y^{2}}{945}-\frac{\zeta_{5}}{y^{3}}+\frac{120}{y^{2}} \operatorname{Re}\left[\mathcal{E}_{0}(6,0,0,0)\right]+\frac{120}{y^{3}} \operatorname{Re}\left[\mathcal{E}_{0}(6,0,0,0,0)\right]\right) \\
&+\pi^{2} s_{13}\left(s_{13}^{2}-s_{12} s_{23}\right)\left(\frac{4 y^{3}}{945}+\frac{2 \zeta_{3}}{5}-\frac{5 \zeta_{5}}{y^{2}}-\frac{3 \zeta_{7}}{2 y^{4}}+\left(\frac{72 \zeta_{3}}{y^{2}}-\frac{16 y}{5}\right) \operatorname{Re}\left[\mathcal{E}_{0}(4,0)\right]\right. \\
&-\frac{12}{5} \operatorname{Re}\left[\mathcal{E}_{0}(4,0,0)\right]-\frac{432}{y^{2}} \operatorname{Re}\left[\mathcal{E}_{0}(4,0)\right] \operatorname{Re}\left[\mathcal{E}_{0}(4,0,0)\right] \\
&+\frac{432}{y^{2}} \operatorname{Re}\left[\mathcal{E}_{0}(4,0,4,0,0)+3 \mathcal{E}_{0}(4,4,0,0,0)+\frac{1}{360} \mathcal{E}_{0}(4,0,0,0,0)\right] \\
&+\frac{4032}{y^{2}} \operatorname{Re}\left[\mathcal{E}_{0}(8,0,0,0,0)\right]+\frac{10080}{y^{3}} \operatorname{Re}\left[\mathcal{E}_{0}(8,0,0,0,0,0)\right] \\
&\left.+\frac{7560}{y^{4}} \operatorname{Re}\left[\mathcal{E}_{0}(8,0,0,0,0,0,0)\right]\right)+\mathcal{O}\left(\alpha^{\prime 4}\right)
\end{aligned}
$$

Up to a global prefactor $(2 \pi i)^{2}$, this expression agrees with the esv image (5.30) of the open-string integral $Z_{1234}^{(2)}$. Hence, we have checked to the order of $\alpha^{\prime 3}$ that

$$
P_{\operatorname{Re}}\left(\mathcal{I}_{1234}^{(2,0)}\left(s_{i j}, \tau\right)\right)=(2 \pi i)^{2} \operatorname{esv} Z_{1234}^{(2)}\left(s_{i j},-\frac{1}{\tau}\right),
$$

and conjecture this relation between open- and closed-string integrals to hold at higher orders as well. In the order- $\alpha^{\prime 3}$ contribution (5.24) to $Z_{1234}^{(2)}\left(s_{i j},-\frac{1}{\tau}\right)$, the product in the third line is understood to be mapped to esv $\left(\mathcal{E}_{0}(4,0) \mathcal{E}_{0}(4,0,0)\right)=\left(\operatorname{esv} \mathcal{E}_{0}(4,0)\right)\left(\operatorname{esv} \mathcal{E}_{0}(4,0,0)\right)$, see (5.30), i.e. without shuffle multiplication prior to the application of esv. Similar adhoc convention are expected to be possible at higher orders of $\mathcal{I}_{1234}^{(2,0)}$ and $Z_{1234}^{(2)}$ such as to satisfy (5.38).

Given that the $\alpha^{\prime}$-expansion of $\mathcal{I}_{1234}^{(2,0)}$ is expressible in terms of modular graph forms, its expansion around the cusp is expected to be of the type (3.44),

$$
\mathcal{I}_{1234}^{(2,0)}\left(s_{i j}, \tau\right)=\sum_{m, n=0}^{\infty} j_{m, n}\left(s_{i j}, y\right) q^{m} \bar{q}^{n} .
$$

The coefficients $j_{m, n}\left(s_{i j}, y\right)$ are series in $s_{i j}$ such that each $\alpha^{\prime}$-order comprises Laurent polynomials in $y$. Since the ambiguities in the evaluation of esv and $P_{\text {Re }}$ were pointed out to be $\mathcal{O}(q \bar{q})$, one can turn (5.38) into a well-defined conjecture by dropping terms $\sim q$,

$$
\mathcal{I}_{1234}^{(2,0)}\left(s_{i j}, \tau\right)=(2 \pi i)^{2} \operatorname{esv} Z_{1234}^{(2)}\left(s_{i j},-\frac{1}{\tau}\right)+\mathcal{O}(q)
$$

This form of our conjecture predicts all the coefficients $j_{0, n}\left(s_{i j}, y\right)$ of $q^{0} \bar{q}^{n}$ in (5.39) with $n \in \mathbb{N}_{0}$ including the zero mode $j_{0,0}\left(s_{i j}, y\right)$ from the open-string quantity $Z_{1234}^{(2)}$. The omission of $\mathcal{O}(q)$-contributions in (5.40) bypasses both the need for the $P_{\text {Re }}$ projection in (5.38) and the incompatibility of esv with the shuffle multiplication. 
The modular weight $(2,0)$ of $\mathcal{I}_{1234}^{(2,0)}\left(s_{i j}, \tau\right)$ is not at all evident from the relations $(5.38)$ and (5.40) with open-string integrals. Hence, it should be possible to infer the coefficients $j_{m, n}\left(s_{i j}, y\right)$ in (5.39) with $m \geq 1$ that do not have any known open-string counterpart from $j_{0, n}\left(s_{i j}, y\right)$ via modular properties. This approach is particularly tractable as long as an ansatz of modular graph forms of suitable transcendental weight is available for a given order in $\alpha^{\prime}$ : for instance, suppose the $\alpha^{\prime 3}$ order of $\mathcal{I}_{1234}^{(2,0)}$ is known to involve a $\mathbb{Q}$-linear combination of $\pi \nabla \mathrm{E}_{4}, \mathrm{E}_{2} \pi \nabla \mathrm{E}_{2}$ and $\pi \nabla \mathrm{E}_{2,2}$, cf. (4.35). Then, the coefficients $c_{1}, c_{2}, c_{3} \in \mathbb{Q}$ in an ansatz

$$
\left.\mathcal{I}_{1234}^{(2,0)}\right|_{\alpha^{\prime 3}}=\frac{s_{13}\left(s_{13}^{2}-s_{12} s_{23}\right)}{(\operatorname{Im} \tau)^{2}}\left(c_{1} \pi \nabla \mathrm{E}_{4}+c_{2} \mathrm{E}_{2} \pi \nabla \mathrm{E}_{2}+c_{3} \pi \nabla \mathrm{E}_{2,2}\right)
$$

are uniquely determined to be $\left(c_{1}, c_{2}, c_{3}\right)=\left(\frac{4}{5}, 6,12\right)$ by (5.38) and (5.40). At the $\alpha^{\prime 4}$-order of $\mathcal{I}_{1234}^{(2,0)}$, one could envision a (4+4)-parameter ansatz comprising $\pi \nabla \mathrm{E}_{5}, \mathrm{E}_{2} \pi \nabla \mathrm{E}_{3}, \mathrm{E}_{3} \pi \nabla \mathrm{E}_{2}$ and $\pi \nabla \mathrm{E}_{2,3}$ (see section 4.2 of [22] for the modular graph function $\mathrm{E}_{2,3}$ ) along with both $s_{12} s_{23} s_{13}^{2}$ and $s_{12}^{4}-4 s_{12}^{2} s_{23}^{2}+s_{23}^{4}$. A more systematic single-valued map extending our present approach involving $P_{\operatorname{Re}}$ will be discussed in [120].

\subsubsection{Integration cycles versus elliptic functions}

It is amusing to compare the single-valued relation between genus-zero integrals in (5.5) with our present evidence for an elliptic single-valued correspondence between open and closed strings. At tree level, the single-valued map of MZVs was found to relate integration cycles $(5.2)$ on a disk boundary to Parke-Taylor factors $\left(z_{12} z_{23} \ldots z_{n 1}\right)^{-1}$. At genus one, the two links (5.28) and (5.38) between open- and closed-string $\alpha^{\prime}$-expansions suggest that integration cycles on a cylinder boundary translate into combinations of the elliptic functions $V_{w}$ in (2.6).

It would be interesting to explain the correspondence between symmetrized open-string cycles and $V_{0}(1,2, \ldots, n)=1$ as well as the four-point cycles of $Z_{1234}^{(2)}$ and $V_{2}(1,2,3,4)$ from the viewpoint of Betti-deRham duality [36, 37]. The general dictionary between $V_{w}(1,2, \ldots, n)$ functions in a closed-string integrand and formal sums of integration cycles $\left\{\left(z_{1}, \ldots, z_{n}\right) \in \mathbb{R}^{n}, 0<z_{1}<z_{2}<\ldots<z_{n}<1\right\}$ on the open-string side will be explored in a sequel of this work [99].

One might wonder if the integral $\mathcal{I}_{1234}^{(4,0)}$ over the elliptic function $V_{4}(1,2,3,4)$ also admits an open-string correspondent along the lines of (5.28) and (5.38). However, the independent permutations of $V_{0}(1,2,3,4)=1$ and $V_{2}(1,2,3,4)$ already exhaust the three combinations of four-point cycles that share the invariance under reflection $z_{j} \rightarrow 1-z_{j}$ of the evenweight $V_{2 k}(1,2,3,4)$. Moreover, since $\mathcal{I}_{1234}^{(4,0)}=\mathrm{G}_{4}\left(1+6 s_{13}\right)+\frac{3 s_{13} \hat{\mathrm{G}}_{2} \pi \nabla \mathrm{E}_{2}}{(\operatorname{Im} \tau)^{2}}+\mathcal{O}\left(\alpha^{\prime 2}\right)$ violates uniform transcendentality, it might be hard to identify a suitable open-string integral with the same property. But it might be a more tractable problem to identify open-string counterparts for the conjecturally uniformly transcendental integrals $\widehat{\mathcal{I}}_{1234}^{(4,0)}, \widehat{\mathcal{I}}_{12 \mid 34}^{(2,0)}$ and $\widehat{\mathcal{I}}_{12 \mid 34}^{(4,0)}$ in (D.13), (D.21) and (D.27), respectively. 


\section{Conclusions}

In this work, we have developed techniques to systematically determine low-energy expansions of one-loop heterotic-string amplitudes. At each order in $\alpha^{\prime}$, the integrations over the punctures are performed at the level of the Fourier modes in a lattice-sum representation of the correlation functions. Consequently, the coefficients in the $\alpha^{\prime}$-expansion are identified to be modular graph forms for any combination of external gravitons and gauge bosons. Explicit results are given for the $\tau$-integrand of the four-point amplitude of non-abelian gauge bosons up to the third subleading order in $\alpha^{\prime}$ - both in the planar and in the nonplanar sector. The integral over the modular parameter up to the second order in $\alpha^{\prime}$ have also been carried out in both cases.

We have furthermore pointed out a striking relation between a specific integral in the planar four-gauge-boson amplitude and a corresponding open-string integral over cylinder punctures. In this way, we provide another incarnation of the idea that closed-string integrals follow from open-string quantities via suitable formal operations - single-valued maps of the periods in the respective $\alpha^{\prime}$-expansions. Our results support a recent proposal for an elliptic single-valued map which arose from inspecting the eMZVs in open-string $\alpha^{\prime}$-expansions and modular graph functions in closed-string $\alpha^{\prime}$-expansions at one loop [22]. The proposal in the reference only applies to situations where the open-string punctures are symmetrized, i.e. independently integrated over the entire cylinder boundary. The heterotic-string integral in this work, by contrast, relates to generic (i.e. non-symmetrized) cyclic orderings on the open-string side under the tentative elliptic single-valued map.

These new connections between four-point open- and closed-string integrals point towards the $n$-point systematics of relating Koba-Nielsen integrals over cylinder and torus punctures. Integration cycles on the cylinder boundaries should translate into elliptic functions in closed-string integrands via Betti-deRham duality $[36,37]$. Since we have identified the open-string integration cycles dual to the elliptic $V_{2}(1,2,3,4)$ function (4.15) of modular weight $(2,0)$, one can expect a similar dictionary for various elliptic $V_{w}$ functions at higher multiplicity [99].

Still, the proposal for an elliptic single-valued map [22] raises several open questions: first, the empirical prescription (5.26) to replace iterated Eisenstein integrals by twice their real parts is incompatible with shuffle multiplication and awaits some reformulation to be given in [120]. Second, it remains to make contact with Brown's construction of singlevalued eMZVs via equivariant iterated Eisenstein integrals [38, 39]. Third, the conjectural relation between open- and closed-string integrals in this work only predicts certain subsectors when expanding closed-string data around the cusp, see (5.40) for details. Given that the main results in this work concern modular graph forms of non-zero modular weights, we hope that they harbor new input on the above open questions.

Some of the Koba-Nielsen integrals in the four-gauge-boson amplitude are found to violate uniform transcendentality in their $\alpha^{\prime}$-expansion. These integrals are rewritten using integration-by-parts relations such as to alleviate certain technical challenges in their $\alpha^{\prime}$ expansion - repeated holomorphic subgraph reduction or regularization of non-absolutely convergent lattice sums. Our integration-by-parts manipulations yield combinations of 
integrals which are individually believed to have a uniformly transcendental $\alpha^{\prime}$-expansion beyond the lowest orders where we have checked this explicitly. On these grounds, our rewritings should be instrumental in constructing a uniform-transcendentality basis for the twisted cohomology for four-point Koba-Nielsen integrals at genus one.

It would be particularly exciting to explore higher-genus generalizations of the present results. Recent progress in the low-energy expansion at genus $g \geq 2$ has been initiated by the identification [121] and the computation [122] of the Zhang-Kawazumi invariant in twoloop amplitudes of type-II superstrings at the subleading order in $\alpha^{\prime}$. Moreover, a highergenus definition of modular graph functions has been recently proposed in [123, 124], along with a comprehensive investigation of their degeneration limits. It would be interesting to extend the analysis of the references to higher-genus modular graph forms, to establish their appearance in heterotic-string amplitudes at $g \geq 2$ loops and to connect with the respective open-string $\alpha^{\prime}$-expansions.

\section{Acknowledgments}

We are grateful to the Hausdorff Center for Mathematics Bonn for providing a stimulating atmosphere, support, and hospitality through the Hausdorff Trimester Program "Periods in Number Theory, Algebraic Geometry and Physics" and the workshop "Amplitudes and Periods". Moreover, we would like to thank Guillaume Bossard, Johannes Broedel, Justin Kaidi, Carlos Mafra, Erik Panzer, Oliver Schnetz and Federico Zerbini for combinations of inspiring discussions and collaboration on related topics. We would like to thank Justin Kaidi, Sebastian Mizera and Federico Zerbini for valuable comments on the manuscript. JG thanks the Perimeter Institute for hospitality during the initial stages of this work. Moreover, JG is supported by the International Max Planck Research School for Mathematical and Physical Aspects of Gravitation, Cosmology and Quantum Field Theory. This research was supported in part by Perimeter Institute for Theoretical Physics. Research at Perimeter Institute is supported by the Government of Canada through the Department of Innovation, Science and Economic Development Canada and by the Province of Ontario through the Ministry of Research, Innovation and Science.

\section{A Relations among modular graph forms}

In this appendix, we present the relations among modular graph forms which are necessary to simplify the heterotic graph forms in the sections 4.2 and 4.3 and comment on some general properties of identities between modular graph forms.

Note that up to the first order in $\alpha^{\prime}$, the identities (3.21) and (3.24) given in the main text are sufficient to simplify the planar heterotic graph forms to single lattice sums. These are also sufficient to simplify the order $\alpha^{\prime 2}$ of $\mathcal{I}_{1234}^{(2,0)}$. For the non-planar heterotic graph forms one needs also the identity (3.7) on top of (3.21) and (3.24), but these relations are then sufficient for all simplifications up to third order in $\alpha^{\prime}$. 


\section{A.1 Relations among dihedral graphs at weight $(4,0)$ and order $\alpha^{\prime 2}$}

The following identities are instrumental in evaluating the $\alpha^{\prime 2}$-order of the integral $\mathcal{I}_{1234}^{(4,0)}$ in (2.43) and can be derived by applying the momentum-conservation identities (3.18) along with (3.20),

$$
\begin{aligned}
\mathcal{C}\left[\begin{array}{lll}
4 & 1 & 1 \\
1 & 1 & 0
\end{array}\right]=-\frac{3}{2} \mathcal{C}\left[\begin{array}{ll}
6 & 0 \\
2 & 0
\end{array}\right]+\frac{1}{2}\left(\mathcal{C}\left[\begin{array}{ll}
3 & 0 \\
1 & 0
\end{array}\right]\right)^{2}, & \mathcal{C}\left[\begin{array}{lll}
4 & 1 & 1 \\
0 & 1 & 1
\end{array}\right]=3 \mathcal{C}\left[\begin{array}{ll}
6 & 0 \\
2 & 0
\end{array}\right]-\left(\mathcal{C}\left[\begin{array}{ll}
3 & 0 \\
1 & 0
\end{array}\right]\right)^{2} \\
\mathcal{C}\left[\begin{array}{lll}
2 & 2 & 2 \\
1 & 1 & 0
\end{array}\right]=0, & \mathcal{C}\left[\begin{array}{lll}
3 & 2 & 1 \\
1 & 1 & 0
\end{array}\right]=\frac{1}{2} \mathcal{C}\left[\begin{array}{ll}
6 & 0 \\
2 & 0
\end{array}\right]-\frac{1}{2}\left(\mathcal{C}\left[\begin{array}{ll}
3 & 0 \\
1 & 0
\end{array}\right]\right)^{2} \\
\mathcal{C}\left[\begin{array}{lll}
3 & 2 & 1 \\
1 & 0 & 1
\end{array}\right]=-\frac{1}{2} \mathcal{C}\left[\begin{array}{ll}
6 & 0 \\
2 & 0
\end{array}\right]+\frac{1}{2}\left(\mathcal{C}\left[\begin{array}{ll}
3 & 0 \\
1 & 0
\end{array}\right]\right)^{2}, & \mathcal{C}\left[\begin{array}{lll}
3 & 2 & 1 \\
0 & 1 & 1
\end{array}\right]=0 .
\end{aligned}
$$

\section{A.2 Relations among dihedral graphs at weight $(2,0)$ and order $\alpha^{\prime 3}$}

In order to perform simplifications at the third order in $\alpha^{\prime}$ of $\mathcal{I}_{1234}^{(2,0)}$, one needs the following identities,

$$
\begin{aligned}
& \mathcal{C}\left[\begin{array}{llll}
2 & 1 & 1 & 1 \\
1 & 1 & 1 & 0
\end{array}\right]=-\mathrm{E}_{2} \mathcal{C}\left[\begin{array}{ll}
3 & 0 \\
1 & 0
\end{array}\right]-2 \mathcal{C}\left[\begin{array}{lll}
3 & 1 & 1 \\
1 & 1 & 1
\end{array}\right]+2 \mathcal{C}\left[\begin{array}{ll}
5 & 0 \\
3 & 0
\end{array}\right] \\
& \mathcal{C}\left[\begin{array}{llll}
2 & 1 & 1 & 1 \\
0 & 1 & 1 & 1
\end{array}\right]=3 \mathrm{E}_{2} \mathcal{C}\left[\begin{array}{ll}
3 & 0 \\
1 & 0
\end{array}\right]+6 \mathcal{C}\left[\begin{array}{lll}
3 & 1 & 1 \\
1 & 1 & 1
\end{array}\right]-6 \mathcal{C}\left[\begin{array}{ll}
5 & 0 \\
3 & 0
\end{array}\right] \\
& \mathcal{C}\left[\begin{array}{lll}
2 & 2 & 1 \\
1 & 1 & 1
\end{array}\right]=0 \\
& \mathcal{C}\left[\begin{array}{lll}
2 & 2 & 1 \\
2 & 0 & 1
\end{array}\right]=-\frac{1}{2} \mathcal{C}\left[\begin{array}{lll}
3 & 1 & 1 \\
1 & 1 & 1
\end{array}\right]+\mathcal{C}\left[\begin{array}{ll}
5 & 0 \\
3 & 0
\end{array}\right] \\
& \mathcal{C}\left[\begin{array}{lll}
2 & 2 & 1 \\
2 & 1 & 0
\end{array}\right]=\frac{1}{2} \mathcal{C}\left[\begin{array}{lll}
3 & 1 & 1 \\
1 & 1 & 1
\end{array}\right]-\mathcal{C}\left[\begin{array}{ll}
5 & 0 \\
3 & 0
\end{array}\right] \\
& \mathcal{C}\left[\begin{array}{lll}
3 & 1 & 1 \\
2 & 1 & 0
\end{array}\right]=-\frac{1}{2} \mathcal{C}\left[\begin{array}{lll}
3 & 1 & 1 \\
1 & 1 & 1
\end{array}\right] \text {. }
\end{aligned}
$$

The first one of these can be verified up to a function of $\bar{\tau}$ of modular weight $(0,-2)$ by acting on both sides with the Cauchy-Riemann operator $\nabla$ in (3.30). The vanishing of the constant follows from the corollary $H_{1234}^{(2,0)}\left[G_{12} G_{13} G_{23}\right]=0$ of (4.30) as can be seen by inserting a trihedral identity from (A.14) and one of the other identities of (A.2) into (B.1). The remaining identities of (A.2) can all be derived from the momentum-conservation identities (3.18) and (3.19).

\section{A.3 Eisenstein regularized sums}

The procedure of holomorphic subgraph reduction [15] is based on performing partialfraction decomposition on the summand of the lattice sum using the momentum of the holomorphic edge. This produces sums of the form

$$
\sum_{\substack{p \neq 0 \\ p \neq q}} \frac{1}{p^{n}}, \quad n \geq 1 .
$$

These can be performed explicitly and hence the original graph is simplified. For $n \geq$ 3, the sums (A.3) are absolutely convergent and evaluate to $\mathrm{G}_{n}-\frac{1}{q^{n}}$. For $n=1,2$, however, (A.3) is not absolutely convergent and hence ill-defined. This ambiguity arises because the original, absolutely convergent, sum was illegally distributed over terms which 
are not absolutely convergent. One can make this step well-defined by first committing to a summation prescription for which all the sums (A.3) are convergent and then distributing the sum. A summation prescription which satisfies this criterion is the so-called Eisenstein summation $\sum_{\mathrm{E}}$ which is defined by $[65,125]$

$$
\sum_{\substack{p \neq 0 \\ p \neq q}} f(p):=\lim _{M \rightarrow \infty} \sum_{\substack{m=-M \\ m \neq 0}}^{M}\left(\lim _{N \rightarrow \infty} \sum_{n=-N}^{N} f(m \tau+n)\right)+\lim _{N \rightarrow \infty} \sum_{\substack{n=-N \\ n \neq 0}}^{N} f(n)-f(q) .
$$

Under Eisenstein summation we obtain (cf. (3.16))

$$
\sum_{\substack{p \neq 0 \\ p \neq q}} \frac{1}{p}=-\frac{1}{q}, \quad \sum_{\substack{p \neq 0 \\ p \neq q}} \frac{1}{p^{2}}=-\frac{1}{q^{2}}+\hat{\mathrm{G}}_{2}+\frac{\pi}{\operatorname{Im} \tau} .
$$

Note that the right-hand side for the sum over $p^{-2}$ is not modular covariant since the contributions $\frac{\pi}{\operatorname{Im} \tau}$ and $\hat{\mathrm{G}}_{2}$ have different modular weights. As explained in the following, these cancel from the final expressions for the present dihedral modular graph forms [15] and also for more general cases of holomorphic subgraph reduction [66]. In the partialfraction decomposition of modular graph forms, also shifted sums of the form

$$
\sum_{\substack{p \neq 0 \\ p \neq q}} \frac{1}{(q-p)^{n}}, \quad n \geq 1
$$

appear. For $n \geq 2$, the shift (and sign flip) of the summation variable is irrelevant. For $n=1$, however, we obtain

$$
\sum_{\substack{p \neq 0 \\ p \neq q}} \frac{1}{q-p}=-\frac{1}{q}-\frac{\pi}{\operatorname{Im} \tau}(q-\bar{q}) .
$$

The right-hand side is again not modular covariant due to the $\frac{\pi}{\operatorname{Im} \tau} q$ term. When assembling the partial-fraction decomposition of modular graph forms, all the terms of the wrong modular weight are found to cancel out, and we are left with modular covariant results. The terms in curly brackets in (3.22) are due to the contributions from $n=1,2$.

Note that in deriving the general expression (3.22), it is convenient to use the functions

$$
Q_{1}(q):=-\frac{1}{q}-\frac{\pi}{2 \operatorname{Im} \tau}(q-\bar{q}), \quad Q_{2}(q):=-\frac{1}{q^{2}}+\hat{\mathrm{G}}_{2}+\frac{\pi}{\operatorname{Im} \tau}
$$

and then to replace

$$
\sum_{\substack{p \neq 0 \\ p \neq q}} \frac{1}{p} \rightarrow Q_{1}(q), \quad \sum_{\substack{p \neq 0 \\ p \neq q}} \frac{1}{q-p} \rightarrow Q_{1}(q), \quad \sum_{\substack{p \neq 0 \\ p \neq q}} \frac{1}{p^{2}} \rightarrow Q_{2}(q) .
$$

In the expressions obtained form partial-fraction decomposition of modular graph forms, this is equivalent to performing the Eisenstein summation as outlined above. 


\section{A.4 Relations among trihedral graphs}

Also for trihedral modular graph forms there exist similar simplification identities as for dihedral modular graph forms. The trihedral momentum-conservation identity is very similar to its dihedral analogue (3.18) and given by [15]

$$
\begin{aligned}
& \sum_{i=1}^{R_{1}} \mathcal{C}\left[\begin{array}{c|c|c}
A_{i} & C & E \\
B & D & F
\end{array}\right]-\sum_{j=1}^{R_{2}} \mathcal{C}\left[\begin{array}{c|c|c}
A & C_{j} & E \\
B & D & F
\end{array}\right]=0 \\
& \sum_{i=1}^{R_{1}} \mathcal{C}\left[\begin{array}{c|c|c}
A & C & E \\
B_{i} & D & F
\end{array}\right]-\sum_{j=1}^{R_{2}} \mathcal{C}\left[\begin{array}{c|c|c}
A & C & E \\
B & D_{j} & F
\end{array}\right]=0,
\end{aligned}
$$

where $A=\left(a_{1}, \ldots, a_{R_{1}}\right)$ and similarly for $B, C, D, E$ and $F$ as in figure 6. Moreover, we are using the shorthand $A_{i}=\left(a_{1}, \ldots, a_{i-1}, a_{i}-1, a_{i+1}, \ldots, a_{R_{1}}\right)$ and similarly for $B_{i}, C_{i}$ and $D_{i}$. Since the order of the blocks in the argument of $\mathcal{C}$ is irrelevant, (A.10) straightforwardly generalizes to any pair of blocks, i.e. with $(C, D) \leftrightarrow(E, F)$ or $(A, B) \leftrightarrow(E, F)$ interchanged.

As in the dihedral case (3.19), whenever an edge carries the trivial decoration $(0,0)$, one summation variable can be removed [15]

$$
\mathcal{C}\left[\begin{array}{ll|l}
A & 0 & C \\
B & 0 & D
\end{array} \mid \underset{F}{E}\right]=(-1)^{|C|+|D|} \mathcal{C}\left[\begin{array}{ll}
C & E \\
D & F
\end{array}\right] \prod_{i=1}^{R_{1}} \mathcal{C}\left[\begin{array}{cc}
a_{i} & 0 \\
b_{i} & 0
\end{array}\right]-\mathcal{C}\left[\begin{array}{l}
A \\
B
\end{array}|\underset{D}{C}| \begin{array}{l}
E \\
F
\end{array}\right] .
$$

For trihedral graphs of the form $\mathcal{C}\left[\begin{array}{l|l|l}0 & C \\ 0 & D & E\end{array}\right]$ one can use (A.11) with the empty product set to one and the replacement

$$
\mathcal{C}[|\underset{D}{C}| \underset{F}{E}]:=\mathcal{C}\left[\begin{array}{l}
C \\
D
\end{array}\right] \mathcal{C}\left[\begin{array}{l}
E \\
F
\end{array}\right]
$$

If two blocks carry just one edge, the trihedral graph simplifies to a dihedral one ${ }^{26}$

$$
\mathcal{C}\left[\begin{array}{l|l|l}
a & c & E \\
b & d & F
\end{array}\right]=(-1)^{a+b+c+d} \mathcal{C}\left[\begin{array}{cc}
a+c & E \\
b+d & F
\end{array}\right] .
$$

To simplify the third order in $\alpha^{\prime}$ of $H_{1234}^{(2,0)}$, we need the trihedral identities

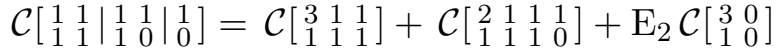

$$
\begin{aligned}
& \mathcal{C}\left[\begin{array}{ll|ll|l}
1 & 1 & 1 & 1 & 1 \\
1 & 0 & 1 & 0 & 1
\end{array}\right]=\frac{1}{2} \mathcal{C}\left[\begin{array}{lll}
3 & 1 & 1 \\
1 & 1 & 1
\end{array}\right]+\frac{1}{2} \mathcal{C}\left[\begin{array}{llll}
2 & 1 & 1 & 1 \\
1 & 1 & 1 & 0
\end{array}\right]+\frac{1}{2} \mathrm{E}_{2} \mathcal{C}\left[\begin{array}{ll}
3 & 0 \\
1 & 0
\end{array}\right],
\end{aligned}
$$

which can be derived from the identities (A.10) and (A.11).

As in the dihedral case, holomorphic subgraph reduction is also possible for trihedral graphs [15]. In this case, the holomorphic subgraph can be either two-valent (i.e. the lattice sum is of the form $\mathcal{C}\left[\begin{array}{ccc|c|c}a_{1} & a_{2} & A & \underset{D}{C} & \underset{D}{0} \\ 0 & 0 & B\end{array}\right]$ ) or three-valent (so that the lattice sum has the form

\footnotetext{
${ }^{26}$ Note that when specializing (A.11) to $\mathcal{C}\left[\begin{array}{cc|c|cc}a & 0 & c & e_{1} & e_{2} \\ b & 0 & d & f_{1} & f_{2}\end{array}\right]$ and using (A.13), there appears to be an overall $(-1)^{c+d}$ difference compared to (7.12) of [15]. This is due to the reversed sign in the $(c, d)$ column used in this section of [15], cf. (7.8) therein.
} 
$\left.\mathcal{C}\left[\begin{array}{cc|cc|cc}a_{1} & A & a_{2} & C & a_{3} & E \\ 0 & B & 0 & D & 0 & F\end{array}\right]\right)$. While the reduction of two-valent subgraphs is a straightforward generalization of dihedral holomorphic subgraph reduction (3.22) and given explicitly in [66], the decomposition of three-valent holomorphic subgraphs yields new sums of the form

$$
\sum_{\substack{p \neq 0 \\ p \neq q_{1}, q_{2}}} \frac{1}{p^{n}}, \quad n \geq 1
$$

generalizing (A.3). As in the dihedral case, one can perform a replacement

$$
\sum_{\substack{p \neq 0 \\ p \neq q_{1}, q_{2}}} \frac{1}{p} \rightarrow Q_{1}\left(q_{1}, q_{2}\right), \quad \sum_{\substack{p \neq 0 \\ p \neq q_{1}, q_{2}}} \frac{1}{q_{1}-p} \rightarrow Q_{1}\left(q_{1}, q_{1}-q_{2}\right), \quad \sum_{\substack{p \neq 0 \\ p \neq q_{1}, q_{2}}} \frac{1}{p^{2}} \rightarrow Q_{2}\left(q_{1}, q_{2}\right)
$$

of the conditionally convergent sums by suitably defined functions [66]

$$
\begin{aligned}
& Q_{1}\left(q_{1}, q_{2}\right):=-\frac{1}{q_{1}}-\frac{1}{q_{2}}-\frac{\pi}{3 \operatorname{Im} \tau}\left(q_{1}+q_{2}-\bar{q}_{1}-\bar{q}_{2}\right) \\
& Q_{2}\left(q_{1}, q_{2}\right):=-\frac{1}{q_{1}^{2}}-\frac{1}{q_{2}^{2}}+\hat{\mathrm{G}}_{2}+\frac{\pi}{\operatorname{Im} \tau} .
\end{aligned}
$$

Once the expressions (A.17) are known, an explicit (though combinatorially involved) formula for trihedral three-valent holomorphic subgraph reduction can be obtained, see [66] for details. Again, the terms of incorrect modular weight due to $Q_{1}$ and $Q_{2}$ in (A.17) cancel out in the final expression.

Using this explicit formula for the trihedral modular graph forms appearing at order $\alpha^{\prime 2}$ in $\mathcal{I}_{1234}^{(4,0)}$, we obtain

$$
\begin{aligned}
\mathcal{C}\left[\begin{array}{ll|l|l}
2 & 1 & 1 & 1 \\
0 & 1 & 1 & 1 \\
0
\end{array}\right]= & -\hat{\mathrm{G}}_{2} \mathcal{C}\left[\begin{array}{ll}
4 & 0 \\
2 & 0
\end{array}\right]-\hat{\mathrm{G}}_{2} \mathcal{C}\left[\begin{array}{lll}
2 & 1 & 1 \\
1 & 1 & 0
\end{array}\right]-\mathcal{C}\left[\begin{array}{ll}
5 & 0 \\
1 & 0
\end{array}\right] \\
& +4 \mathcal{C}\left[\begin{array}{ll}
6 & 0 \\
2 & 0
\end{array}\right]-\mathcal{C}\left[\begin{array}{lll}
3 & 1 & 1 \\
0 & 0 & 1
\end{array}\right]-\mathcal{C}\left[\begin{array}{lll}
3 & 2 & 1 \\
0 & 1 & 1
\end{array}\right]+3 \mathcal{C}\left[\begin{array}{lll}
4 & 1 & 1 \\
1 & 1 & 0
\end{array}\right] \\
\mathcal{C}\left[\begin{array}{ll|l|l}
1 & 1 & 1 & 1 \\
1 & 0 & 1 & 0 \\
0
\end{array}\right]= & 2 \hat{\mathrm{G}}_{2} \mathcal{C}\left[\begin{array}{ll}
4 & 0 \\
2 & 0
\end{array}\right]+2 \hat{\mathrm{G}}_{2} \mathcal{C}\left[\begin{array}{lll}
2 & 1 & 1 \\
1 & 1 & 0
\end{array}\right]+2 \mathcal{C}\left[\begin{array}{ll}
5 & 0 \\
1 & 0
\end{array}\right] \\
& -6 \mathcal{C}\left[\begin{array}{ll}
6 & 0 \\
2 & 0
\end{array}\right]+2 \mathcal{C}\left[\begin{array}{lll}
3 & 1 & 1 \\
0 & 0 & 1
\end{array}\right]+2 \mathcal{C}\left[\begin{array}{lll}
3 & 2 & 1 \\
1 & 0 & 1
\end{array}\right]-6 \mathcal{C}\left[\begin{array}{lll}
4 & 1 & 1 \\
1 & 1 & 0
\end{array}\right] .
\end{aligned}
$$

Simplifying these using the identities from section A.1 as well as the dihedral holomorphic subgraph relation (3.24) yields the trihedral decompositions of (4.25).

\section{A.5 Tetrahedral holomorphic subgraph reduction}

The tetrahedral graph $\mathcal{C}_{\text {tet }}$ defined in (4.23) can be decomposed using tetrahedral holomorphic subgraph reduction. It is convenient to employ the following partial-fraction decomposition of the sum (4.23) w.r.t. $p_{3}$,

$$
\begin{aligned}
\mathcal{C}_{\text {tet }}= & \left(\frac{\operatorname{Im} \tau}{\pi}\right)^{2} \sum_{\substack{p_{1}, p_{2}, p_{3} \neq 0 \\
p_{1}+p_{3} \neq 0 \\
p_{2}+p_{3} \neq 0 \\
p_{1}+p_{2}+p_{3} \neq 0 \\
p_{1} \neq \pm p_{2}}} \frac{1}{\left|p_{1}\right|^{2}\left|p_{2}\right|^{2} p_{3}\left(p_{1}+p_{3}\right)\left(p_{2}+p_{3}\right)\left(p_{1}+p_{2}+p_{3}\right)}+\mathcal{C}_{\mathrm{tet}}^{p_{1}=p_{2}}+\mathcal{C}_{\mathrm{tet}}^{p_{1}=-p_{2}} \\
= & \left(\frac{\operatorname{Im} \tau}{\pi}\right)_{\substack{p_{1}, p_{2}, p_{3} \neq 0 \\
p_{1}+p_{3} \neq 0 \\
p_{2}+p_{3} \neq 0 \\
p_{1}+p_{2}+p_{3} \neq 0 \\
p_{1} \neq \pm p_{2}}}^{2} \frac{1}{\left|p_{1}\right|^{2}\left|p_{2}\right|^{2}}\left[\frac{1}{p_{1}\left(p_{1}-p_{2}\right) p_{2}\left(p_{1}+p_{3}\right)}-\frac{1}{p_{1}\left(p_{1}-p_{2}\right) p_{2}\left(p_{2}+p_{3}\right)}\right.
\end{aligned}
$$


where $\mathcal{C}_{\text {tet }}^{p_{1}= \pm p_{2}}$ is the sum (4.23) with $p_{1}$ set to $\pm p_{2}$. The terms $\mathcal{C}_{\text {tet }}^{p_{1}= \pm p_{2}}$ involving fewer momenta are of lower complexity and can be simplified using the techniques of section A.4. The $p_{3}$-sum in the remaining contributions to (A.19) can be performed by means of the replacements

$$
\sum_{\substack{p \neq 0 \\ p \neq q_{1}, q_{2}, q_{3}}} \frac{1}{p} \rightarrow Q_{1}\left(q_{1}, q_{2}, q_{3}\right), \quad \sum_{\substack{p \neq 0 \\ p \neq-q_{1}, q_{2}, q_{3}}} \frac{1}{p+q_{1}} \rightarrow Q_{1}\left(q_{1}, q_{1}+q_{2}, q_{1}+q_{3}\right),
$$

where $[66]$

$$
Q_{1}\left(q_{1}, q_{2}, q_{3}\right):=-\frac{1}{q_{1}}-\frac{1}{q_{2}}-\frac{1}{q_{3}}-\frac{\pi}{4 \operatorname{Im} \tau}\left(q_{1}+q_{2}+q_{3}-\bar{q}_{1}-\bar{q}_{2}-\bar{q}_{3}\right) .
$$

Putting everything together, (A.19) can be written in terms of dihedral modular graph forms,

$$
\mathcal{C}_{\text {tet }}=-2 \hat{\mathrm{G}}_{2} \mathcal{C}\left[\begin{array}{ll}
4 & 0 \\
2 & 0
\end{array}\right]+2 \mathcal{C}\left[\begin{array}{ll}
6 & 0 \\
2 & 0
\end{array}\right]+4 \mathcal{C}\left[\begin{array}{lll}
2 & 1 & 2 \\
0 & 0 & 1
\end{array}\right]+4 \mathcal{C}\left[\begin{array}{lll}
2 & 2 & 2 \\
1 & 1 & 0
\end{array}\right]-8 \mathcal{C}\left[\begin{array}{lll}
3 & 2 & 1 \\
1 & 1 & 0
\end{array}\right]
$$

After using (3.24) and the identities from section A.1, this simplifies to the last line in (4.25).

\section{B The $V_{2}(1,2,3,4)$ integral at the third order in $\alpha^{\prime}$}

In this appendix, we list the heterotic graph forms contributing to $\mathcal{I}_{1234}^{(2,0)}$ at order $\alpha^{\prime 3}$.

\section{B.1 The twelve inequivalent heterotic graph forms}

Table 1 lists the twelve inequivalent heterotic graph forms at order $\alpha^{\prime 3}$ in the planar sector. The contributions of modular weight $(2,0)$ are explicitly given by

$$
\begin{aligned}
& H_{1234}^{(2,0)}\left[G_{12}^{3}\right]=-\mathcal{C}\left[\begin{array}{llll}
2 & 1 & 1 & 1 \\
0 & 1 & 1 & 1
\end{array}\right] \\
& H_{1234}^{(2,0)}\left[G_{13}^{3}\right]=2 \mathcal{C}\left[\begin{array}{llll}
2 & 1 & 1 & 1 \\
0 & 1 & 1 & 1
\end{array}\right]
\end{aligned}
$$

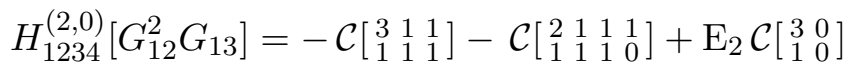

$$
\begin{aligned}
& H_{1234}^{(2,0)}\left[G_{12}^{2} G_{14}\right]=\mathcal{C}\left[\begin{array}{lll}
3 & 1 & 1 \\
1 & 1 & 1
\end{array}\right]-\mathrm{E}_{2} \mathcal{C}\left[\begin{array}{ll}
3 & 0 \\
1 & 0
\end{array}\right] \\
& H_{1234}^{(2,0)}\left[G_{12}^{2} G_{34}\right]=\mathcal{C}\left[\begin{array}{lll}
3 & 1 & 1 \\
1 & 1 & 1
\end{array}\right]-\mathrm{E}_{2} \mathcal{C}\left[\begin{array}{ll}
3 & 0 \\
1 & 0
\end{array}\right] \\
& H_{1234}^{(2,0)}\left[G_{13}^{2} G_{12}\right]=-\mathcal{C}\left[\begin{array}{lll}
3 & 1 & 1 \\
1 & 1 & 1
\end{array}\right]+\mathcal{C}\left[\begin{array}{ll|l|l}
1 & 1 & 1 & 1 \\
1 & 1 & 1 & 0 \\
1 & 0
\end{array}\right]-\mathrm{E}_{2} \mathcal{C}\left[\begin{array}{lll}
3 & 0 \\
1 & 0
\end{array}\right] \\
& H_{1234}^{(2,0)}\left[G_{13}^{2} G_{24}\right]=-2 \mathcal{C}\left[\begin{array}{lll}
3 & 1 & 1 \\
1 & 1 & 1
\end{array}\right]+2 \mathrm{E}_{2} \mathcal{C}\left[\begin{array}{ll}
3 & 0 \\
1 & 0
\end{array}\right] \\
& H_{1234}^{(2,0)}\left[G_{12} G_{13} G_{14}\right]=-\mathcal{C}\left[\begin{array}{lll}
2 & 2 & 1 \\
1 & 1 & 1
\end{array}\right] \\
& H_{1234}^{(2,0)}\left[G_{12} G_{13} G_{23}\right]=-\mathcal{C}\left[\begin{array}{lll}
2 & 2 & 1 \\
2 & 0 & 1
\end{array}\right]+\mathcal{C}\left[\begin{array}{lllll}
1 & 1 & 1 & 1 & 1 \\
1 & 0 & 1 & 1 \\
1 & 0 & 1
\end{array}\right] \\
& H_{1234}^{(2,0)}\left[G_{12} G_{13} G_{24}\right]=-\mathcal{C}\left[\begin{array}{ll}
5 & 0 \\
3 & 0
\end{array}\right]+\mathcal{C}\left[\begin{array}{lll}
2 & 2 & 1 \\
1 & 1 & 1
\end{array}\right]-2 \mathcal{C}\left[\begin{array}{lll}
2 & 2 & 1 \\
2 & 1 & 0
\end{array}\right]+\mathcal{C}\left[\begin{array}{lll}
3 & 1 & 1 \\
2 & 1 & 0
\end{array}\right] \\
& H_{1234}^{(2,0)}\left[G_{12} G_{13} G_{34}\right]=-\mathcal{C}\left[\begin{array}{lll}
2 & 2 & 1 \\
1 & 1 & 1
\end{array}\right] \\
& H_{1234}^{(2,0)}\left[G_{12} G_{14} G_{23}\right]=-\mathcal{C}\left[\begin{array}{ll}
5 & 0 \\
3 & 0
\end{array}\right]-3 \mathcal{C}\left[\begin{array}{lll}
3 & 1 & 1 \\
2 & 1 & 0
\end{array}\right] \text {. }
\end{aligned}
$$




\section{B.2 Identities among modular graph forms at order $\alpha^{\prime 3}$}

Using the relations from appendix A, the heterotic graph forms (B.1) can be simplified. We obtain e.g.

$$
\begin{aligned}
H_{1234}^{(2,0)}\left[G_{12}^{3}\right] & =-6 \mathcal{C}\left[\begin{array}{lll}
3 & 1 & 1 \\
1 & 1 & 1
\end{array}\right]+6 \mathcal{C}\left[\begin{array}{ll}
5 & 0 \\
3 & 0
\end{array}\right]-3 \mathrm{E}_{2} \mathcal{C}\left[\begin{array}{ll}
3 & 0 \\
1 & 0
\end{array}\right] \\
H_{1234}^{(2,0)}\left[G_{12}^{2} G_{13}\right] & =\mathcal{C}\left[\begin{array}{lll}
3 & 1 & 1 \\
1 & 1 & 1
\end{array}\right]-2 \mathcal{C}\left[\begin{array}{ll}
5 & 0 \\
3 & 0
\end{array}\right]+2 \mathrm{E}_{2} \mathcal{C}\left[\begin{array}{ll}
3 & 0 \\
1 & 0
\end{array}\right] \\
H_{1234}^{(2,0)}\left[G_{12}^{2} G_{14}\right] & =\mathcal{C}\left[\begin{array}{lll}
3 & 1 & 1 \\
1 & 1 & 1
\end{array}\right]-\mathrm{E}_{2} \mathcal{C}\left[\begin{array}{ll}
3 & 0 \\
1 & 0
\end{array}\right] \\
H_{1234}^{(2,0)}\left[G_{12} G_{14} G_{23}\right] & =\frac{3}{2} \mathcal{C}\left[\begin{array}{lll}
3 & 1 & 1 \\
1 & 1 & 1
\end{array}\right]-\mathcal{C}\left[\begin{array}{ll}
5 & 0 \\
3 & 0
\end{array}\right] .
\end{aligned}
$$

Once this simplification is performed for all heterotic graph forms in (B.1), it is clear that the remaining contributions in (B.1) are either related to those in (B.2) or zero,

$$
\begin{aligned}
H_{1234}^{(2,0)}\left[G_{13}^{3}\right] & =-2 H_{1234}^{(2,0)}\left[G_{12}^{3}\right] \\
H_{1234}^{(2,0)}\left[G_{12}^{2} G_{34}\right] & =H_{1234}^{(2,0)}\left[G_{12}^{2} G_{14}\right] \\
H_{1234}^{(2,0)}\left[G_{13}^{2} G_{12}\right] & =-H_{1234}^{(2,0)}\left[G_{12}^{2} G_{13}\right]-H_{1234}^{(2,0)}\left[G_{12}^{2} G_{14}\right] \\
H_{1234}^{(2,0)}\left[G_{13}^{2} G_{24}\right] & =-2 H_{1234}^{(2,0)}\left[G_{12}^{2} G_{14}\right] \\
H_{1234}^{(2,0)}\left[G_{12} G_{13} G_{24}\right] & =-H_{1234}^{(2,0)}\left[G_{12} G_{14} G_{23}\right] \\
H_{1234}^{(2,0)}\left[G_{12} G_{13} G_{14}\right] & =H_{1234}^{(2,0)}\left[G_{12} G_{13} G_{23}\right]=H_{1234}^{(2,0)}\left[G_{12} G_{13} G_{34}\right]=0 .
\end{aligned}
$$

Note that the expressions (B.3) are compatible with the symmetries (4.30).

\section{The $V_{2}(i, j)$ integrals at the third order in $\alpha^{\prime}$}

In this appendix, we list the heterotic graph forms contributing to the double-trace sector at order $\alpha^{\prime 3}$.

\section{C.1 The inequivalent heterotic graph forms of weight $(2,0)$}

Table 2 lists the twelve inequivalent heterotic graph forms at order $\alpha^{\prime 3}$ in the non-planar sector. Using the regularized values given in (4.43) and (4.47), the weight- $(2,0)$ contributions are given by

$$
\begin{aligned}
H_{12 \mid 34}^{(2,0)}\left[G_{12}^{3}\right] & =\hat{\mathrm{G}}_{2}\left(2\left(\mathrm{E}_{3}+\zeta_{3}-3\right)-3 \mathrm{E}_{2}\right)+12 \mathcal{C}\left[\begin{array}{ll}
3 & 0 \\
1 & 0
\end{array}\right]-6 \mathcal{C}\left[\begin{array}{ll}
4 & 0 \\
2 & 0
\end{array}\right] \\
H_{12 \mid 34}^{(2,0)}\left[G_{13}^{3}\right] & =2 \hat{\mathrm{G}}_{2}\left(\mathrm{E}_{3}+\zeta_{3}\right) \\
H_{12 \mid 34}^{(2,0)}\left[G_{12}^{2} G_{34}\right] & =-\mathrm{E}_{2} \hat{\mathrm{G}}_{2} \\
H_{12 \mid 34}^{(2,0)}\left[G_{12} G_{13}^{2}\right] & =-\mathrm{E}_{2} \hat{\mathrm{G}}_{2} \\
H_{12 \mid 34}^{(2,0)}\left[G_{13}^{2} G_{14}\right] & =-\mathcal{C}\left[\begin{array}{ll}
4 & 0 \\
2 & 0
\end{array}\right] \\
H_{12 \mid 34}^{(2,0)}\left[G_{12} G_{13} G_{24}\right] & =-\mathcal{C}\left[\begin{array}{ll}
4 & 0 \\
2 & 0
\end{array}\right]
\end{aligned}
$$




$$
\begin{aligned}
H_{12 \mid 34}^{(2,0)}\left[G_{13} G_{14} G_{34}\right] & =2 \mathrm{E}_{3} \hat{\mathrm{G}}_{2}+\mathcal{C}\left[\begin{array}{ll}
3 & 0 \\
1 & 0
\end{array}\right]-\frac{5}{2} \mathcal{C}\left[\begin{array}{cc}
4 & 0 \\
2 & 0
\end{array}\right] \\
H_{12 \mid 34}^{(2,0)}\left[G_{12}^{2} G_{13}\right] & =H_{12 \mid 34}^{(2,0)}\left[G_{12} G_{13} G_{34}\right]=H_{12 \mid 34}^{(2,0)}\left[G_{12} G_{13} G_{14}\right] \\
& =H_{12 \mid 34}^{(2,0)}\left[G_{13}^{2} G_{24}\right]=H_{12 \mid 34}^{(2,0)}\left[G_{13} G_{14} G_{23}\right]=0 .
\end{aligned}
$$

Note that the appearance of $\zeta_{3}$ is due to the modular graph function $C_{1,1,1}$ in intermediate steps, see (3.7).

\section{C.2 The inequivalent heterotic graph forms of weight $(4,0)$}

Similarly to section C.1, the regularized values of the weight- $(4,0)$ contributions are given by

$$
\begin{aligned}
& H_{12 \mid 34}^{(4,0)}\left[G_{12}^{3}\right]=\hat{\mathrm{G}}_{2}^{2}\left(\mathrm{E}_{3}-3 \mathrm{E}_{2}+\zeta_{3}-6\right)+12 \hat{\mathrm{G}}_{2} \mathcal{C}\left[\begin{array}{ll}
3 & 0 \\
1 & 0
\end{array}\right]-6 \hat{\mathrm{G}}_{2} \mathcal{C}\left[\begin{array}{ll}
4 & 0 \\
2 & 0
\end{array}\right] \\
& H_{12 \mid 34}^{(4,0)}\left[G_{13}^{3}\right]=\hat{\mathrm{G}}_{2}^{2}\left(\mathrm{E}_{3}+\zeta_{3}\right) \\
& H_{12 \mid 34}^{(4,0)}\left[G_{12}^{2} G_{34}\right]=-\left(2+\mathrm{E}_{2}\right) \hat{\mathrm{G}}_{2}^{2}+4 \hat{\mathrm{G}}_{2} \mathcal{C}\left[\begin{array}{ll}
3 & 0 \\
1 & 0
\end{array}\right] \\
& H_{12 \mid 34}^{(4,0)}\left[G_{12} G_{13}^{2}\right]=-\mathrm{E}_{2} \hat{\mathrm{G}}_{2}^{2} \\
& H_{12 \mid 34}^{(4,0)}\left[G_{13}^{2} G_{14}\right]=-\hat{\mathrm{G}}_{2} \mathcal{C}\left[\begin{array}{cc}
4 & 0 \\
2 & 0
\end{array}\right] \\
& H_{12 \mid 34}^{(4,0)}\left[G_{12} G_{13} G_{14}\right]=\hat{\mathrm{G}}_{2} \mathcal{C}\left[\begin{array}{ll}
3 & 0 \\
1 & 0
\end{array}\right] \\
& H_{12 \mid 34}^{(4,0)}\left[G_{13}^{2} G_{24}\right]=-2 \mathrm{G}_{4}-2 \hat{\mathrm{G}}_{2} \mathcal{C}\left[\begin{array}{ll}
3 & 0 \\
1 & 0
\end{array}\right]+8 \mathcal{C}\left[\begin{array}{ll}
5 & 0 \\
1 & 0
\end{array}\right] \\
& H_{12 \mid 34}^{(4,0)}\left[G_{12} G_{13} G_{24}\right]=-2 \mathrm{G}_{4}-\hat{\mathrm{G}}_{2} \mathcal{C}\left[\begin{array}{ll}
3 & 0 \\
1 & 0
\end{array}\right]-\hat{\mathrm{G}}_{2} \mathcal{C}\left[\begin{array}{ll}
4 & 0 \\
2 & 0
\end{array}\right]+6 \mathcal{C}\left[\begin{array}{ll}
5 & 0 \\
1 & 0
\end{array}\right] \\
& H_{12 \mid 34}^{(4,0)}\left[G_{13} G_{14} G_{34}\right]=\mathrm{E}_{3} \hat{\mathrm{G}}_{2}^{2}+\hat{\mathrm{G}}_{2} \mathcal{C}\left[\begin{array}{ll}
3 & 0 \\
1 & 0
\end{array}\right]-\frac{5}{2} \hat{\mathrm{G}}_{2} \mathcal{C}\left[\begin{array}{ll}
4 & 0 \\
2 & 0
\end{array}\right] \\
& H_{12 \mid 34}^{(4,0)}\left[G_{13} G_{14} G_{23}\right]=2 \mathrm{G}_{4}+2 \hat{\mathrm{G}}_{2} \mathcal{C}\left[\begin{array}{ll}
3 & 0 \\
1 & 0
\end{array}\right]-6 \mathcal{C}\left[\begin{array}{ll}
5 & 0 \\
1 & 0
\end{array}\right] \\
& H_{12 \mid 34}^{(4,0)}\left[G_{12}^{2} G_{13}\right]=H_{12 \mid 34}^{(4,0)}\left[G_{12}^{2} G_{13}\right]=0 .
\end{aligned}
$$

\section{Decomposition into uniform-transcendentality integrals}

In this appendix, we give more details on the rearrangements of the integrals in section 4.4 and in particular derive the expressions for the conjecturally uniformly transcendental integrals $\widehat{\mathcal{I}}_{\ldots}^{(w, 0)}$ via integrations by parts.

The notion of transcendental weight for modular graph forms can be derived from the terminology for eMZVs $(5.8)$, where $\omega\left(n_{1}, n_{2}, \ldots, n_{r}\right)$ is said to have weight $n_{1}+n_{2}+\ldots+n_{r}$. This convention implies weight one for $\pi$, weight $n_{1}+n_{2}+\ldots+n_{r}$ for $\zeta_{n_{1}, n_{2}, \ldots, n_{r}}$ and weight $r$ for iterated Eisenstein integrals $\mathcal{E}_{0}\left(k_{1}, k_{2}, \ldots, k_{r}\right)$ in (3.38). This means that both types of Eisenstein series $\mathrm{G}_{k}$ and $\mathrm{E}_{k}$ have weight $k$ and that $\pi \nabla$ as well as $y=\pi \operatorname{Im} \tau$ have weight one. Similarly, $(\pi \nabla)^{p} \mathrm{E}_{k}$ and $(\pi \nabla)^{p} \mathrm{E}_{2,2}$ are found to carry weight $k+p$ and $4+p$, respectively.

\section{D.1 Planar integrals}

In this section, we provide a decomposition of $\mathcal{I}_{1234}^{(4,0)}$ defined in (2.43) into the integrals of uniform transcendentality. In particular, we derive (4.50). 


\section{D.1.1 Integration-by-parts manipulations}

The idea is to exploit the fact that total derivatives $\partial_{i}=\frac{\partial}{\partial z_{i}}$ of the Koba-Nielsen factor (with $\partial_{i} G_{i j}=-f_{i j}^{(1)}$ )

$$
\mathcal{J}_{n}:=\prod_{i<j}^{n} \exp \left(s_{i j} G_{i j}(\tau)\right), \quad \partial_{i} \mathcal{J}_{n}=-\mathcal{J}_{n} \sum_{j \neq i}^{n} s_{i j} f_{i j}^{(1)}
$$

integrate to zero. In order to relate this to the constituents $f^{(w)}$ of the integrand $V_{4}(1,2,3,4)$ of $\mathcal{I}_{1234}^{(4,0)}$, cf. $(4.15)$, the total $z_{i}$-derivatives have to furthermore act on suitably chosen functions of modular weight three. As we will see, the identities of interest involve the combination $^{27}$

$$
\begin{aligned}
X_{1234}^{(3)}= & f_{12}^{(1)} f_{23}^{(1)} f_{34}^{(1)}+\frac{1}{6}\left(f_{12}^{(3)}+f_{34}^{(3)}\right)+\frac{2}{3} f_{23}^{(3)}+\frac{1}{3} f_{23}^{(1)}\left(f_{12}^{(2)}+f_{34}^{(2)}\right) \\
& +\frac{2}{3} f_{23}^{(2)}\left(f_{12}^{(1)}+f_{34}^{(1)}\right)+\frac{1}{2}\left(f_{12}^{(1)} f_{34}^{(2)}+f_{12}^{(2)} f_{34}^{(1)}\right)
\end{aligned}
$$

whose derivatives in $z_{1}, \ldots, z_{4}$ can be evaluated using

$$
\begin{aligned}
& \partial_{z} f^{(1)}(z)=2 f^{(2)}(z)-\left(f^{(1)}(z)\right)^{2}-\hat{\mathrm{G}}_{2} \\
& \partial_{z} f^{(2)}(z)=3 f^{(3)}(z)-f^{(1)}(z) f^{(2)}(z)-\hat{\mathrm{G}}_{2} f^{(1)}(z) \\
& \partial_{z} f^{(3)}(z)=4 f^{(4)}(z)-f^{(1)}(z) f^{(3)}(z)-\hat{\mathrm{G}}_{2} f^{(2)}(z)-\mathrm{G}_{4} .
\end{aligned}
$$

The virtue of the combination (D.2) is that it allows generating $V_{4}(1,2,3,4)$ and simpler elliptic functions by means of total derivatives in the punctures as detailed below. Indeed, by the symmetries $X_{1234}^{(3)}=-X_{4321}^{(3)}$ and $X_{1234}^{(3)}+X_{2134}^{(3)}+X_{2314}^{(3)}+X_{2341}^{(3)}=0$ due to Fay identities [6, 49], one can show that

$$
\partial_{4} X_{1234}^{(3)}+\partial_{2} X_{1432}^{(3)}-\partial_{3}\left(X_{1423}^{(3)}+X_{1243}^{(3)}\right)=\mathrm{G}_{4}+\hat{\mathrm{G}}_{2} V_{2}(1,2,3,4)-V_{4}(1,2,3,4) .
$$

In order to relate this to the Koba-Nielsen integral $\mathcal{I}_{1234}^{(4,0)}$, we extend (D.4) to the following total derivative via (D.1) and $V_{4}(1,2,3,4)+\operatorname{cyc}(2,3,4)=3 \mathrm{G}_{4}$,

$$
\begin{aligned}
& \partial_{4}\left(X_{1234}^{(3)} \mathcal{J}_{4}\right)+\partial_{2}\left(X_{1432}^{(3)} \mathcal{J}_{4}\right)-\partial_{3}\left(X_{1243}^{(3)} \mathcal{J}_{4}\right)-\partial_{3}\left(X_{1423}^{(3)} \mathcal{J}_{4}\right) \\
& =\mathcal{J}_{4}\left[\hat{\mathrm{G}}_{2} V_{2}(1,2,3,4)-\left(1+s_{1234}\right) V_{4}(1,2,3,4)+\mathrm{G}_{4}+3\left(s_{13}+s_{24}\right) \mathrm{G}_{4}+\widehat{V}_{4}(1,2,3,4)\right],
\end{aligned}
$$

using the shorthands $s_{1234}:=s_{12}+s_{13}+s_{14}+s_{23}+s_{24}+s_{34}$ and

$$
\begin{aligned}
\widehat{V}_{4}(1,2,3,4)= & s_{12} R_{2|34| 1}+s_{23} R_{3|41| 2}+s_{34} R_{4|12| 3}+s_{14} R_{1|23| 4} \\
& -s_{13}\left(R_{1|24| 3}+R_{1|42| 3}\right)-s_{24}\left(R_{2|13| 4}+R_{2|31| 4}\right)
\end{aligned}
$$

comprising several permutations of

$$
R_{1|23| 4}=f_{14}^{(1)} X_{1234}^{(3)}+V_{4}(1,2,3,4) .
$$

\footnotetext{
${ }^{27}$ OS would like to thank Carlos Mafra for collaboration on related topics, where the meromorphic version of (D.2) with all the $\operatorname{Im} z_{j}$ removed has been studied.
} 
In the remainder of this subsection, we elaborate on some of the intermediate steps in (D.5): when the derivatives act on the Koba-Nielsen factor $\mathcal{J}_{4}$, they generate the terms

$$
\begin{aligned}
& X_{1234}^{(3)}\left(s_{14} f_{14}^{(1)}+s_{24} f_{24}^{(1)}+s_{34} f_{34}^{(1)}\right)+X_{1432}^{(3)}\left(s_{12} f_{12}^{(1)}-s_{23} f_{23}^{(1)}-s_{24} f_{24}^{(1)}\right) \\
& -\left(X_{1243}^{(3)}+X_{1423}^{(3)}\right)\left(s_{13} f_{13}^{(1)}+s_{23} f_{23}^{(1)}-s_{34} f_{34}^{(1)}\right) \\
& =\left[s_{14} f_{14}^{(1)} X_{1234}^{(3)}+\operatorname{cyc}(1,2,3,4)\right]-s_{13} f_{13}^{(1)}\left(X_{1243}^{(3)}+X_{1423}^{(3)}\right)-s_{24} f_{24}^{(1)}\left(X_{2134}^{(3)}+X_{2314}^{(3)}\right),
\end{aligned}
$$

where the symmetries $X_{1234}^{(3)}=-X_{4321}^{(3)}$ and $X_{1234}^{(3)}+X_{2134}^{(3)}+X_{2314}^{(3)}+X_{2341}^{(3)}=0$ have been used in passing to the last line. However, we have refrained from using momentum conservation in (D.5) or (D.8) so far. The term $s_{1234} V_{4}(1,2,3,4)$ in the second line of (D.5) has been generated by rewriting each term in (D.8) via permutations of (D.7). The coefficients of $s_{13}$ and $s_{24}$ in (D.8) require the additional intermediate step

$$
\begin{aligned}
-f_{13}^{(1)}\left(X_{1243}^{(3)}+X_{1423}^{(3)}\right) & =V_{4}(1,2,4,3)+V_{4}(1,4,2,3)-R_{1|24| 3}-R_{1|42| 3} \\
& =3 \mathrm{G}_{4}-V_{4}(1,2,3,4)-R_{1|24| 3}-R_{1|42| 3}
\end{aligned}
$$

in reproducing (D.5).

\section{D.1.2 Uniform transcendentality decomposition}

Using the representation (D.2) and (4.15) of its constituents $X_{1234}^{(3)}$ and $V_{4}(1,2,3,4), R_{1|23| 4}$ can be expanded as

$$
\begin{aligned}
R_{1|23| 4}= & f_{23}^{(1)} f_{34}^{(1)} f_{12}^{(2)}+f_{12}^{(1)} f_{34}^{(1)} f_{23}^{(2)}+f_{12}^{(1)} f_{23}^{(1)} f_{34}^{(2)}+f_{12}^{(1)} f_{23}^{(1)} f_{14}^{(2)}+f_{12}^{(1)} f_{34}^{(1)} f_{14}^{(2)}+f_{23}^{(1)} f_{34}^{(1)} f_{14}^{(2)} \\
& -\frac{1}{3} f_{12}^{(1)} f_{14}^{(1)} f_{23}^{(2)}-\frac{1}{3} f_{14}^{(1)} f_{34}^{(1)} f_{23}^{(2)}-\frac{2}{3} f_{14}^{(1)} f_{23}^{(1)} f_{12}^{(2)}-\frac{2}{3} f_{14}^{(1)} f_{23}^{(1)} f_{34}^{(2)}-\frac{1}{2} f_{14}^{(1)} f_{34}^{(1)} f_{12}^{(2)} \\
& -\frac{1}{2} f_{12}^{(1)} f_{14}^{(1)} f_{34}^{(2)}+f_{12}^{(2)} f_{14}^{(2)}+f_{12}^{(2)} f_{23}^{(2)}+f_{14}^{(2)} f_{23}^{(2)}+f_{12}^{(2)} f_{34}^{(2)}+f_{14}^{(2)} f_{34}^{(2)}+f_{23}^{(2)} f_{34}^{(2)} \quad(\mathrm{D} .10) \\
& -\frac{5}{6} f_{14}^{(1)} f_{12}^{(3)}-\frac{5}{6} f_{14}^{(1)} f_{34}^{(3)}-\frac{1}{3} f_{14}^{(1)} f_{23}^{(3)}-f_{12}^{(1)} f_{14}^{(3)}-f_{23}^{(1)} f_{14}^{(3)}-f_{34}^{(1)} f_{14}^{(3)}+f_{23}^{(1)} f_{12}^{(3)} \\
& +f_{23}^{(1)} f_{34}^{(3)}+f_{34}^{(1)} f_{12}^{(3)}+f_{12}^{(1)} f_{34}^{(3)}+f_{12}^{(1)} f_{23}^{(3)}+f_{34}^{(1)} f_{23}^{(3)}+f_{12}^{(4)}+f_{14}^{(4)}+f_{23}^{(4)}+f_{34}^{(4)}
\end{aligned}
$$

Hence, all the terms of $R_{1|23| 4}$ involve at most three factors of $f_{i j}^{(n)}$, and none of them exhibits a subcycle $f_{i j}^{(m)} f_{j i}^{(n)}$ or $f_{i j}^{(m)} f_{j k}^{(n)} f_{k i}^{(p)}$. This means none of the resulting graphs in the order-by-order integration against monomials in $G_{i j}$ contains closed holomorphic subgraphs and hence the need for holomorphic subgraph reduction is removed.

Apart from $R_{1|23| 4}=R_{4|32| 1}$, there are no further relations among the 12 reflectionindependent permutations of $R_{1|23| 4}$ in (D.7). The reflection property is sufficient to show that $\widehat{V}_{4}(1,2,3,4)+\operatorname{cyc}(2,3,4)=0$.

In the momentum phase-space of four particles with $s_{13}=s_{24}$ and $s_{1234}=0$, one can solve (D.5) for

$$
V_{4}(1,2,3,4) \cong \hat{\mathrm{G}}_{2} V_{2}(1,2,3,4)+\mathrm{G}_{4}+6 s_{13} \mathrm{G}_{4}+\widehat{V}_{4}(1,2,3,4),
$$

where the equivalence relation $\cong$ indicates that total derivatives $\partial_{i}\left(\ldots \mathcal{J}_{4}\right)$ have to be discarded in equating $\left(\mathcal{J}_{4}\right.$ times) the two sides of (D.11). At the level of the integrals, this implies

$$
\mathcal{I}_{1234}^{(4,0)}\left(s_{i j}, \tau\right)=\mathrm{G}_{4} \mathcal{I}_{1234}^{(0,0)}\left(s_{i j}, \tau\right)+\hat{\mathrm{G}}_{2} \mathcal{I}_{1234}^{(2,0)}\left(s_{i j}, \tau\right)+\widehat{\mathcal{I}}_{1234}^{(4,0)}\left(s_{i j}, \tau\right),
$$


cf. (4.51), where we have introduced a new Koba-Nielsen integral

$$
\begin{aligned}
& \widehat{\mathcal{I}}_{1234}^{(4,0)}\left(s_{i j}, \tau\right)=\int \mathrm{d} \mu_{4} \exp \left(\sum_{1 \leq i<j}^{4} s_{i j} G_{i j}(\tau)\right)\left\{6 s_{13} \mathrm{G}_{4}+s_{12} R_{2|34| 1}+s_{23} R_{3|41| 2}\right. \\
& \left.\quad+s_{34} R_{4|12| 3}+s_{14} R_{1|23| 4}-s_{13}\left(R_{1|24| 3}+R_{1|42| 3}\right)-s_{24}\left(R_{2|13| 4}+R_{2|31| 4}\right)\right\}
\end{aligned}
$$

Given that the $R_{i|j k| l}$ boil down to the $f^{(w)}$ with a lattice-sum representation (4.2), the coefficients in the $\alpha^{\prime}$-expansion of (D.13) are guaranteed to be modular graph forms by the arguments of section 4.1. By the absence of subcycles in the constituents (D.7), the $\alpha^{\prime}$-expansion of $\widehat{\mathcal{I}}_{1234}^{(4,0)}$ is expected to exhibit uniform transcendentality with weight $k+3$ at the order of $\alpha^{\prime k}$. This is confirmed by the leading orders in $\alpha^{\prime}$,

$$
\widehat{\mathcal{I}}_{1234}^{(4,0)}\left(s_{i j}, \tau\right)=6 s_{13} \mathrm{G}_{4}+2\left(s_{13}^{2}+2 s_{12} s_{23}\right) \mathcal{C}\left[\begin{array}{ll}
5 & 0 \\
1 & 0
\end{array}\right]+\mathcal{O}\left(\alpha^{\prime 3}\right),
$$

as can be seen from the form given in (4.51).

By inserting the decomposition (D.12) of the integral $\mathcal{I}_{1234}^{(4,0)}$ into (2.42), we arrive at the decomposition of the complete planar $\tau$-integrand as stated in (4.50).

\section{D.1.3 Consistency check of the leading contributions to $\widehat{\mathcal{I}}_{1234}^{(4,0)}$}

The leading-order result (D.14) can not only been obtained from (D.12) but can also be checked in an independent calculation based on an $\alpha^{\prime}$-expansion of (D.13) as follows. We use the notation

$$
\mathcal{R}_{a|b c| d}\left[\prod_{i<j} G_{i j}^{n_{i j}}\right]=\int \mathrm{d} \mu_{4} R_{a|b c| d} \prod_{i<j} G_{i j}^{n_{i j}}
$$

analogous to (4.12). In the absence of closed subcycles in the expression (D.10) for $R_{1|23| 4}$, the leading order evidently vanishes,

$$
\mathcal{R}_{a|b c| d}[\emptyset]=0 .
$$

At the subleading order in $\alpha^{\prime}$, the same representation of $R_{1|23| 4}$ yields

$$
\begin{array}{lll}
\mathcal{R}_{1|23| 4}\left[G_{12}\right]=-3 \mathcal{C}\left[\begin{array}{ll}
5 & 0 \\
1 & 0
\end{array}\right], & \mathcal{R}_{1|23| 4}\left[G_{13}\right]=\frac{35}{6} \mathcal{C}\left[\begin{array}{ll}
5 & 0 \\
1 & 0
\end{array}\right], & \mathcal{R}_{1|23| 4}\left[G_{14}\right]=-4 \mathcal{C}\left[\begin{array}{ll}
5 & 0 \\
1 & 0
\end{array}\right] \\
\mathcal{R}_{1|23| 4}\left[G_{23}\right]=-3 \mathcal{C}\left[\begin{array}{ll}
5 & 0 \\
1 & 0
\end{array}\right], & \mathcal{R}_{1|23| 4}\left[G_{24}\right]=\frac{35}{6} \mathcal{C}\left[\begin{array}{ll}
5 & 0 \\
1 & 0
\end{array}\right], & \mathcal{R}_{1|23| 4}\left[G_{34}\right]=-3 \mathcal{C}\left[\begin{array}{ll}
5 & 0 \\
1 & 0
\end{array}\right],
\end{array}
$$

and any other $\mathcal{R}_{a|b c| d}\left[G_{i j}\right]$ can be obtained by relabeling. Finally, the contribution of $6 s_{13} \mathrm{G}_{4}$ in (D.13) integrates to $6 s_{13} \mathrm{G}_{4} \mathcal{I}^{(0,0)}$ with $\mathcal{I}^{(0,0)}=1+\mathcal{O}\left(\alpha^{\prime 2}\right)$, completing the verification of (D.14) to the orders shown.

\section{D.2 Non-planar integrals}

The non-planar integrals $\mathcal{I}_{12 \mid 34}^{(2,0)}$ and $\mathcal{I}_{12 \mid 34}^{(4,0)}$ in (2.46) and (2.47) admit integration-by-parts manipulations analogous to (D.11) to be rewritten in terms of (conjecturally) uniformly transcendental integrals $\widehat{\mathcal{I}}_{12 \mid 34}^{(w, 0)}$. In this section we derive these decompositions, given in (4.54) in the main text. 
In the non-planar cases, the total derivatives are simpler and boil down to iterations of

$$
\partial_{2}\left(f_{12}^{(1)} \mathcal{J}_{4}\right)=\mathcal{J}_{4}\left[\hat{\mathrm{G}}_{2}-\left(1+s_{12}\right) V_{2}(1,2)+2 s_{12} f_{12}^{(2)}-f_{12}^{(1)}\left(s_{23} f_{23}^{(1)}+s_{24} f_{24}^{(1)}\right)\right]
$$

which can be used to solve for

$$
\left(1+s_{12}\right) V_{2}(1,2) \cong \hat{\mathrm{G}}_{2}+2 s_{12} f_{12}^{(2)}-f_{12}^{(1)}\left(s_{23} f_{23}^{(1)}+s_{24} f_{24}^{(1)}\right)
$$

Again, $\cong$ indicates that total derivatives $\partial_{i}\left(\ldots \mathcal{J}_{4}\right)$ have been discarded in passing to the right-hand side. A similar identity can be derived by taking a $z_{1}$-derivative in (D.18), so the right-hand side of (D.19) turns out to be symmetric under the simultaneous exchange of $\left(z_{1}, s_{1 j}\right) \leftrightarrow\left(z_{2}, s_{2 j}\right)$, at least up to total derivatives.

\section{D.2.1 Rewriting the integral $\mathcal{I}_{12 \mid 34}^{(2,0)}$}

By applying (D.19) to both summands $V_{2}(1,2)$ and $V_{2}(3,4)$ of $(2.47)$, one arrives at a decomposition

$$
\mathcal{I}_{12 \mid 34}^{(2,0)}\left(s_{i j}, \tau\right)=\frac{2 \hat{\mathrm{G}}_{2} \mathcal{I}^{(0,0)}\left(s_{i j}, \tau\right)+\widehat{\mathcal{I}}_{12 \mid 34}^{(2,0)}\left(s_{i j}, \tau\right)}{1+s_{12}}
$$

involving a new integral that should be uniformly transcendental,

$$
\begin{aligned}
\widehat{\mathcal{I}}_{12 \mid 34}^{(2,0)}\left(s_{i j}, \tau\right)= & \int \mathrm{d} \mu_{4} \exp \left(\sum_{1 \leq i<j}^{4} s_{i j} G_{i j}(\tau)\right) \\
& \times\left[2 s_{12} f_{12}^{(2)}-f_{12}^{(1)}\left(s_{23} f_{23}^{(1)}+s_{24} f_{24}^{(1)}\right)+2 s_{34} f_{34}^{(2)}-f_{34}^{(1)}\left(s_{41} f_{41}^{(1)}+s_{42} f_{42}^{(1)}\right)\right] \\
= & 2 \int \mathrm{d} \mu_{4} \exp \left(\sum_{1 \leq i<j}^{4} s_{i j} G_{i j}(\tau)\right)\left[2 s_{12} f_{12}^{(2)}-f_{12}^{(1)}\left(s_{23} f_{23}^{(1)}+s_{24} f_{24}^{(1)}\right)\right]
\end{aligned}
$$

In passing to the last line, we have used Mandelstam identities and the symmetries of the Koba-Nielsen factor to obtain identities such as

$$
\int \mathrm{d} \mu_{4} \exp \left(\sum_{1 \leq i<j}^{4} s_{i j} G_{i j}(\tau)\right) s_{23} f_{12}^{(1)} f_{23}^{(1)}=\int \mathrm{d} \mu_{4} \exp \left(\sum_{1 \leq i<j}^{4} s_{i j} G_{i j}(\tau)\right) s_{14} f_{34}^{(1)} f_{41}^{(1)}
$$

The expansion of $\widehat{\mathcal{I}}_{12 \mid 34}^{(2,0)}$ up to the third order in $\alpha^{\prime}$ is given in (4.55) and verified to be uniformly transcendental. 


\section{D.2.2 Rewriting the integral $\mathcal{I}_{12 \mid 34}^{(4,0)}$}

Repeated application of (D.18) leads to a total-derivative relation for the integrand of $\mathcal{I}_{12 \mid 34}^{(4,0)}$,

$$
\begin{aligned}
(1+ & \left.s_{12}\right)\left(1+s_{34}\right) V_{2}(1,2) V_{2}(3,4) \cong \hat{\mathrm{G}}_{2}^{2}+s_{13}^{2} f_{12}^{(1)} f_{24}^{(1)} f_{43}^{(1)} f_{31}^{(1)}+s_{14}^{2} f_{12}^{(1)} f_{23}^{(1)} f_{34}^{(1)} f_{41}^{(1)} \\
& +\hat{\mathrm{G}}_{2}\left[2 s_{12} f_{12}^{(2)}+2 s_{34} f_{34}^{(2)}+\frac{1}{2} f_{12}^{(1)}\left(s_{13} f_{13}^{(1)}+s_{14} f_{14}^{(1)}-s_{23} f_{23}^{(1)}-s_{24} f_{24}^{(1)}\right)\right. \\
& \left.\quad+\frac{1}{2} f_{34}^{(1)}\left(s_{24} f_{24}^{(1)}+s_{14} f_{14}^{(1)}-s_{23} f_{23}^{(1)}-s_{13} f_{13}^{(1)}\right)\right]+4 s_{12} s_{34} f_{12}^{(2)} f_{34}^{(2)} \\
& \quad+s_{34} f_{34}^{(2)} f_{12}^{(1)}\left(s_{13} f_{13}^{(1)}+s_{14} f_{14}^{(1)}-s_{23} f_{23}^{(1)}-s_{24} f_{24}^{(1)}\right) \\
& +s_{12} f_{12}^{(2)} f_{34}^{(1)}\left(s_{24} f_{24}^{(1)}+s_{14} f_{14}^{(1)}-s_{23} f_{23}^{(1)}-s_{13} f_{13}^{(1)}\right)
\end{aligned}
$$

and therefore to a similar decomposition for the integral as in (D.20):

$$
\mathcal{I}_{12 \mid 34}^{(4,0)}\left(s_{i j}, \tau\right)=\frac{\hat{\mathrm{G}}_{2}^{2} \mathcal{I}^{(0,0)}\left(s_{i j}, \tau\right)+\hat{\mathrm{G}}_{2} \widehat{\mathcal{I}}_{12 \mid 34}^{(2,0)}\left(s_{i j}, \tau\right)+\breve{\mathcal{I}}_{12 \mid 34}^{(4,0)}\left(s_{i j}, \tau\right)}{\left(1+s_{12}\right)^{2}} .
$$

Here, we have introduced the integral

$$
\begin{array}{r}
\check{\mathcal{I}}_{12 \mid 34}^{(4,0)}\left(s_{i j}, \tau\right)=\int \mathrm{d} \mu_{4} \exp \left(\sum_{1 \leq i<j}^{4} s_{i j} G_{i j}(\tau)\right)\left[4 s_{12} s_{34} f_{12}^{(2)} f_{34}^{(2)}+4 s_{34} f_{34}^{(2)} f_{12}^{(1)}\left(s_{13} f_{13}^{(1)}+s_{14} f_{14}^{(1)}\right)\right. \\
\left.+s_{13}^{2} f_{12}^{(1)} f_{24}^{(1)} f_{43}^{(1)} f_{31}^{(1)}+s_{14}^{2} f_{12}^{(1)} f_{23}^{(1)} f_{34}^{(1)} f_{41}^{(1)}\right]
\end{array}
$$

over the terms without $\hat{\mathrm{G}}_{2}$ on the right-hand side of (D.23), where relabeling identities of the form (D.22) were used to simplify the result. Again, we have independently verified (D.20) and (D.24) to the order of $\alpha^{\prime 3}$ by expanding the integrals in (D.21) and (D.25) along the lines of section D.1.3. Upon insertion into (2.42), we arrive at an alternative representation of the non-planar sector of the four-point amplitude,

$$
\begin{aligned}
\left.M_{4}(\tau)\right|_{\operatorname{Tr}\left(t^{a_{1}} t^{a_{2}}\right) \operatorname{Tr}\left(t^{a_{3}} t^{a_{4}}\right)}= & \frac{\mathrm{G}_{4}^{2} \breve{\mathcal{I}}_{12 \mid 34}^{(4,0)}}{\left(1+s_{12}\right)^{2}}+\left(\frac{\mathrm{G}_{4}^{2} \hat{\mathrm{G}}_{2}}{\left(1+s_{12}\right)^{2}}-\frac{7}{2} \frac{\mathrm{G}_{4} \mathrm{G}_{6}}{1+s_{12}}\right) \widehat{\mathcal{I}}_{12 \mid 34}^{(2,0)} \\
& +\left(\frac{\mathrm{G}_{4}^{2} \hat{\mathrm{G}}_{2}^{2}}{\left(1+s_{12}\right)^{2}}-7 \frac{\mathrm{G}_{4} \mathrm{G}_{6} \hat{\mathrm{G}}_{2}}{1+s_{12}}+\frac{5}{3} \mathrm{G}_{4}^{3}+\frac{49}{6} \mathrm{G}_{6}^{2}\right) \mathcal{I}^{(0,0)}
\end{aligned}
$$

\section{D.2.3 Towards a uniform-transcendentality basis}

However, this is not yet the desired uniform-transcendentality decomposition since the last line in the integrand (D.25) of $\breve{\mathcal{I}}_{12 \mid 34}^{(4,0)}$ exhibits closed subcycles $f_{i j}^{(1)} f_{j k}^{(1)} f_{k l}^{(1)} f_{l i}^{(1)}$. One can isolate a piece of uniform transcendentality from (D.25) by subtracting these subcycles via $V_{4}(i, j, k, l)$, i.e. the integral

$$
\begin{gathered}
\widehat{\mathcal{I}}_{12 \mid 34}^{(4,0)}\left(s_{i j}, \tau\right)=\int \mathrm{d} \mu_{4} \exp \left(\sum_{1 \leq i<j}^{4} s_{i j} G_{i j}(\tau)\right)\left[4 s_{12} s_{34} f_{12}^{(2)} f_{34}^{(2)}+4 s_{34} f_{34}^{(2)} f_{12}^{(1)}\left(s_{13} f_{13}^{(1)}+s_{14} f_{14}^{(1)}\right)\right. \\
\left.+s_{13}^{2}\left(f_{12}^{(1)} f_{24}^{(1)} f_{43}^{(1)} f_{31}^{(1)}-V_{4}(1,2,4,3)\right)+s_{14}^{2}\left(f_{12}^{(1)} f_{23}^{(1)} f_{34}^{(1)} f_{41}^{(1)}-V_{4}(1,2,3,4)\right)\right]
\end{gathered}
$$


is claimed to be uniformly transcendental. Then, by the decomposition (D.12) of integrals over $V_{4}(i, j, k, l)$, we can relate this to (D.25) via

$$
\check{\mathcal{I}}_{12 \mid 34}^{(4,0)}=\widehat{\mathcal{I}}_{12 \mid 34}^{(4,0)}+\left(s_{13}^{2}+s_{23}^{2}\right) \mathrm{G}_{4} \mathcal{I}^{(0,0)}+s_{13}^{2}\left(\widehat{\mathcal{I}}_{1243}^{(4,0)}+\hat{\mathrm{G}}_{2} \mathcal{I}_{1243}^{(2,0)}\right)+s_{23}^{2}\left(\widehat{\mathcal{I}}_{1234}^{(4,0)}+\hat{\mathrm{G}}_{2} \mathcal{I}_{1234}^{(2,0)}\right) .
$$

Upon insertion into (D.26), we obtain admixtures of the planar integrals $\widehat{\mathcal{I}}_{1234}^{(4,0)}$ and $\mathcal{I}_{1234}^{(2,0)}$ defined by (D.13) and (2.44), respectively, and arrive at (4.54). Similar to section D.1.3, the results for $\mathcal{I}_{12 \mid 34}^{(w, 0)}$ in (4.48) and (4.49) have been confirmed by performing an independent $\alpha^{\prime}$ expansion of (D.21) and (D.27) and inserting into the above integration-by-parts relations.

\section{D.3 Efficiency of the new representations for higher-order expansions}

Given the Mandelstam invariants in the integrands (D.13), (D.21) and (D.27) of $\widehat{\mathcal{I}}_{1234}^{(4,0)}$, $\widehat{\mathcal{I}}_{12 \mid 34}^{(2,0)}$ and $\widehat{\mathcal{I}}_{12 \mid 34}^{(4,0)}$, the $k^{\text {th }}$ order in their $\alpha^{\prime}$-expansion can be computed from less than $k$ factors of $G_{i j}$ from the Koba-Nielsen factor. However, the variety of $f_{i j}^{(w)}$ along with the different $s_{k l}$ in the integrands increases the number of independent calculations w.r.t. relabeling the punctures and momenta at a fixed order of the Koba-Nielsen expansion. Hence, the combinatorial efficiency of the new representations (4.50) and (4.54) of $M_{4}(\tau)$ for higher-order $\alpha^{\prime}$-expansion should be comparable to the old one in (2.42).

Instead, the main advantages of the integrals $\widehat{\mathcal{I}}_{1234}^{(4,0)}, \widehat{\mathcal{I}}_{12 \mid 34}^{(2,0)}$ and $\widehat{\mathcal{I}}_{12 \mid 34}^{(4,0)}$ are the following:

- The integrand of $\widehat{\mathcal{I}}_{1234}^{(4,0)}$ in (D.13) does not share the term $f_{12}^{(1)} f_{23}^{(1)} f_{34}^{(1)} f_{41}^{(1)}$ of the $V_{4}(1,2,3,4)$ function. Like this, expansion of $\widehat{\mathcal{I}}_{1234}^{(4,0)}$ bypasses numerous holomorphic subgraph reductions that introduced a spurious complexity into the calculations of section 4.2 .

- The $\alpha^{\prime}$-expansions of $\mathcal{I}_{12 \mid 34}^{(w, 0)}$ in section 4.3 were plagued by conditionally convergent or divergent lattice sums. Lack of absolute convergence is caused by the terms $\left(f_{i j}^{(1)}\right)^{2}$ or $\sum_{p \neq 0} \frac{\bar{p}}{p} e^{2 \pi i\left\langle p, z_{i j}\right\rangle}$ in the representations (4.37) or (4.9) of $V_{2}(i, j)$. Both of them are manifestly absent in the integrands (D.21) and (D.27) of $\widehat{\mathcal{I}}_{12 \mid 34}^{(2,0)}$ and $\widehat{\mathcal{I}}_{12 \mid 34}^{(4,0)}$.

\section{E The elliptic single-valued map at the third order in $\alpha^{\prime}$}

In this appendix, we give some further explicit expressions necessary to relate the $\left(\alpha^{\prime}\right)^{3}$ order of open- and closed-string integrals through the tentative elliptic single-valued map in section 5 .

\section{E.1 Modular transformation of iterated Eisenstein integrals}

Modular transformations of the iterated Eisenstein integrals at the orders $\alpha^{\prime}$ and $\alpha^{\prime 2}$ of (5.19) were given in (5.20). The order $\alpha^{\prime 3}$ also necessitates the modular transforma- 
tions $[22,69]$

$$
\begin{aligned}
& \mathcal{E}_{0}\left(8,0,0,0,0 ;-\frac{1}{\tau}\right)=-\frac{i \pi^{2} T^{3}}{95256000}+\frac{i \pi^{6}}{13608000 T}+\frac{i \pi^{8}}{2857680 T^{3}}+\frac{\pi^{2} \zeta_{7}}{672 T^{4}}-\frac{i \pi^{10}}{4536000 T^{5}} \\
& +\frac{\pi^{2}}{T^{2}} \mathcal{E}_{0}(8,0,0,0,0)+\frac{5 i \pi^{2}}{T^{3}} \mathcal{E}_{0}(8,0,0,0,0,0)-\frac{15 \pi^{2}}{2 T^{4}} \mathcal{E}_{0}(8,0,0,0,0,0,0) \\
& \mathcal{E}_{0}\left(4,0,4,0,0 ;-\frac{1}{\tau}\right)+3 \mathcal{E}_{0}\left(4,4,0,0,0 ;-\frac{1}{\tau}\right)+\frac{1}{360} \mathcal{E}_{0}\left(4,0,0,0,0 ;-\frac{1}{\tau}\right)=\frac{i \pi^{2} T^{3}}{2916000}+\frac{i \pi^{4} T}{116640} \\
& \quad+\frac{5 \zeta_{5}}{432}-\frac{\pi^{2} \zeta_{3}}{6480}-\frac{91 i \pi^{6}}{1458000 T}-\frac{5 \pi^{2} \zeta_{5}}{432 T^{2}}-\frac{\pi^{4} \zeta_{3}}{1296 T^{2}}+\frac{i \pi^{8}}{38880 T^{3}}+\frac{i \pi^{2} \zeta_{3}^{2}}{72 T^{3}} \\
& \quad+\frac{\pi^{6} \zeta_{3}}{2160 T^{4}}-\frac{i \pi^{10}}{324000 T^{5}}+\left(\frac{\pi^{2}}{1080}+\frac{\pi^{4}}{216 T^{2}}-\frac{i \pi^{2} \zeta_{3}}{6 T^{3}}-\frac{\pi^{6}}{360 T^{4}}\right) \mathcal{E}_{0}(4,0,0) \\
& \quad+\frac{i \pi^{2}}{2 T^{3}} \mathcal{E}_{0}(4,0,0)^{2}+\frac{\pi^{2}}{T^{2}}\left(\mathcal{E}_{0}(4,0,4,0,0)+3 \mathcal{E}_{0}(4,4,0,0,0)+\frac{\mathcal{E}_{0}(4,0,0,0,0)}{360}\right) .
\end{aligned}
$$

\section{E.2 The third $\alpha^{\prime}$-order of $\mathcal{I}_{1234}^{(2,0)}$ and iterated Eisenstein integrals}

The first and second order of $\mathcal{I}_{1234}^{(2,0)}$ in $\alpha^{\prime}$ were written in terms of iterated Eisenstein integrals in (5.32). Similarly, the third order of the expansion in (4.35) can be written as

$$
\begin{aligned}
& \left.\mathcal{I}_{1234}^{(2,0)}\right|_{\alpha^{\prime 3}}=\pi^{2} s_{13}\left(s_{12}^{2}+s_{12} s_{23}+s_{23}^{2}\right)\left(\frac{4 y^{3}}{945}+\frac{2 \zeta_{3}}{5}-\frac{5 \zeta_{5}}{y^{2}}-\frac{3 \zeta_{7}}{2 y^{4}}+\left(\frac{72 \zeta_{3}}{y^{2}}-\frac{16 y}{5}\right) \operatorname{Re}\left[\mathcal{E}_{0}(4,0)\right]\right. \\
& -\frac{12}{5} \operatorname{Re}\left[\mathcal{E}_{0}(4,0,0)\right]+\frac{432}{y^{2}} \operatorname{Re}\left[\mathcal{E}_{0}(4,0,4,0,0)+3 \mathcal{E}_{0}(4,4,0,0,0)+\frac{1}{360} \mathcal{E}_{0}(4,0,0,0,0)\right] \\
& +\frac{4032}{y^{2}} \operatorname{Re}\left[\mathcal{E}_{0}(8,0,0,0,0)\right]+\frac{10080}{y^{3}} \operatorname{Re}\left[\mathcal{E}_{0}(8,0,0,0,0,0)\right]+\frac{7560}{y^{4}} \operatorname{Re}\left[\mathcal{E}_{0}(8,0,0,0,0,0,0)\right] \\
& -\frac{432}{y^{2}} \operatorname{Re}\left[\mathcal{E}_{0}(4,0)\right] \operatorname{Re}\left[\mathcal{E}_{0}(4,0,0)\right]+\left(\frac{16 y^{2}}{5}+\frac{144 \zeta_{3}}{y}\right) \mathcal{E}_{0}(4)+\frac{24}{5} y \mathcal{E}_{0}(4,0)+\frac{24}{5} \mathcal{E}_{0}(4,0,0) \\
& +\frac{432}{y} \mathcal{E}_{0}(4,0)^{2}-1728 \mathcal{E}_{0}(4) \operatorname{Re}\left[\mathcal{E}_{0}(4,0)\right]-\frac{864}{y} \mathcal{E}_{0}(4,0) \operatorname{Re}\left[\mathcal{E}_{0}(4,0)\right]-\frac{864}{y} \mathcal{E}_{0}(4) \operatorname{Re}\left[\mathcal{E}_{0}(4,0,0)\right] \\
& +1728 \mathcal{E}_{0}(4,4,0)+\frac{864}{y}\left[\mathcal{E}_{0}(4,4,0,0)+\frac{1}{360} \mathcal{E}_{0}(4,0,0,0)\right]+2688 \mathcal{E}_{0}(8,0,0) \\
& \left.+\frac{8064}{y} \mathcal{E}_{0}(8,0,0,0)+\frac{10080}{y^{2}} \mathcal{E}_{0}(8,0,0,0,0)+\frac{5040}{y^{3}} \mathcal{E}_{0}(8,0,0,0,0,0)\right) .
\end{aligned}
$$

As in (5.32), not all of these terms are reproduced by $\left.(2 \pi i)^{2} \operatorname{esv} Z_{1234}^{(2)}\left(s_{i j},-\frac{1}{\tau}\right)\right|_{\alpha^{\prime}}$, but only the ones which are left invariant under the projection $P_{\mathrm{Re}}$ defined in (5.33). Specifically, $P_{\operatorname{Re}}$ maps the terms $\left(\frac{16 y^{2}}{5}+\frac{144 \zeta_{3}}{y}\right) \mathcal{E}_{0}(4)+\frac{24}{5} y \mathcal{E}_{0}(4,0)+\frac{24}{5} \mathcal{E}_{0}(4,0,0)$ in the fourth line and all terms in the following lines to zero.

Open Access. This article is distributed under the terms of the Creative Commons Attribution License (CC-BY 4.0), which permits any use, distribution and reproduction in any medium, provided the original author(s) and source are credited.

\section{References}

[1] T. Terasoma, Selberg integrals and multiple zeta values, Compos. Math. 133 (2002) 1.

[2] F. Brown, Multiple zeta values and periods of moduli spaces $\mathcal{M}_{0, n}(\mathbb{R})$, Annales Sci. Ecole Norm. Sup. 42 (2009) 371 [math/0606419]. 
[3] O. Schlotterer and S. Stieberger, Motivic Multiple Zeta Values and Superstring Amplitudes, J. Phys. A 46 (2013) 475401 [arXiv:1205.1516] [InSPIRE].

[4] J. Broedel, O. Schlotterer, S. Stieberger and T. Terasoma, All order $\alpha^{\prime}$-expansion of superstring trees from the Drinfeld associator, Phys. Rev. D 89 (2014) 066014 [arXiv:1304.7304] [INSPIRE].

[5] B. Enriquez, Analogues elliptiques des nombres multizétas, Bull. Soc. Math. Fr. 144 (2016) 395 [arXiv: 1301.3042].

[6] J. Broedel, C.R. Mafra, N. Matthes and O. Schlotterer, Elliptic multiple zeta values and one-loop superstring amplitudes, JHEP 07 (2015) 112 [arXiv:1412.5535] [INSPIRE].

[7] J. Broedel, N. Matthes, G. Richter and O. Schlotterer, Twisted elliptic multiple zeta values and non-planar one-loop open-string amplitudes, J. Phys. A 51 (2018) 285401 [arXiv: 1704.03449] [INSPIRE].

[8] E. D'Hoker, M.B. Green, Ö. Gürdoğan and P. Vanhove, Modular Graph Functions, Commun. Num. Theor. Phys. 11 (2017) 165 [arXiv:1512.06779] [INSPIRE].

[9] M.B. Green and P. Vanhove, The low-energy expansion of the one loop type-II superstring amplitude, Phys. Rev. D 61 (2000) 104011 [hep-th/9910056] [INSPIRE].

[10] M.B. Green, J.G. Russo and P. Vanhove, Low energy expansion of the four-particle genus-one amplitude in type-II superstring theory, JHEP 02 (2008) 020 [arXiv:0801.0322] [INSPIRE].

[11] E. D'Hoker, M.B. Green and P. Vanhove, On the modular structure of the genus-one Type II superstring low energy expansion, JHEP 08 (2015) 041 [arXiv: 1502.06698] [INSPIRE].

[12] E. D'Hoker, M.B. Green and P. Vanhove, Proof of a modular relation between 1-, 2- and 3-loop Feynman diagrams on a torus, J. Number Theory 196 (2019) 381 [arXiv:1509.00363] [INSPIRE].

[13] A. Basu, Poisson equation for the Mercedes diagram in string theory at genus one, Class. Quant. Grav. 33 (2016) 055005 [arXiv:1511.07455] [INSPIRE].

[14] F. Zerbini, Single-valued multiple zeta values in genus 1 superstring amplitudes, Commun. Num. Theor. Phys. 10 (2016) 703 [arXiv:1512.05689] [INSPIRE].

[15] E. D'Hoker and M.B. Green, Identities between Modular Graph Forms, J. Number Theor. 189 (2018) 25 [arXiv:1603.00839] [INSPIRE].

[16] A. Basu, Poisson equation for the three loop ladder diagram in string theory at genus one, Int. J. Mod. Phys. A 31 (2016) 1650169 [arXiv:1606.02203] [INSPIRE].

[17] A. Basu, Proving relations between modular graph functions, Class. Quant. Grav. 33 (2016) 235011 [arXiv: 1606.07084] [INSPIRE].

[18] A. Basu, Simplifying the one loop five graviton amplitude in type IIB string theory, Int. J. Mod. Phys. A 32 (2017) 1750074 [arXiv:1608.02056] [InSPIRE].

[19] E. D'Hoker and J. Kaidi, Hierarchy of Modular Graph Identities, JHEP 11 (2016) 051 [arXiv: 1608.04393] [INSPIRE].

[20] A. Kleinschmidt and V. Verschinin, Tetrahedral modular graph functions, JHEP 09 (2017) 155 [arXiv: 1706.01889 ] [INSPIRE].

[21] E. D'Hoker and W. Duke, Fourier series of modular graph functions, arXiv:1708.07998 [INSPIRE]. 
[22] J. Broedel, O. Schlotterer and F. Zerbini, From elliptic multiple zeta values to modular graph functions: open and closed strings at one loop, arXiv:1803.00527 [INSPIRE].

[23] W. Lerche, B.E.W. Nilsson, A.N. Schellekens and N.P. Warner, Anomaly Cancelling Terms From the Elliptic Genus, Nucl. Phys. B 299 (1988) 91 [inSPIRE].

[24] S. Stieberger and T.R. Taylor, NonAbelian Born-Infeld action and type I-heterotic duality (II): Nonrenormalization theorems, Nucl. Phys. B 648 (2003) 3 [hep-th/0209064] [INSPIRE].

[25] L. Dolan and P. Goddard, Current Algebra on the Torus, Commun. Math. Phys. 285 (2009) 219 [arXiv: 0710.3743] [INSPIRE].

[26] A. Basu, Low momentum expansion of one loop amplitudes in heterotic string theory, JHEP 11 (2017) 139 [arXiv: 1708.08409] [INSPIRE].

[27] A. Basu, A simplifying feature of the heterotic one loop four graviton amplitude, Phys. Lett. B 776 (2018) 182 [arXiv:1710.01993] [INSPIRE].

[28] S. Stieberger, Closed superstring amplitudes, single-valued multiple zeta values and the Deligne associator, J. Phys. A 47 (2014) 155401 [arXiv:1310.3259] [INSPIRE].

[29] S. Stieberger and T.R. Taylor, Closed String Amplitudes as Single-Valued Open String Amplitudes, Nucl. Phys. B 881 (2014) 269 [arXiv:1401.1218] [INSPIRE].

[30] F. Brown and C. Dupont, Superstring amplitudes in genus 0 and 1, talk given by F. Brown in String Math, Sendai, Japan, June 18, 2018.

[31] O. Schlotterer and O. Schnetz, Closed strings as single-valued open strings: A genus-zero derivation, arXiv:1808.00713 [INSPIRE].

[32] F. Brown and C. Dupont, Single-valued integration and superstring amplitudes in genus zero, arXiv: 1810.07682 [INSPIRE].

[33] O. Schnetz, Graphical functions and single-valued multiple polylogarithms, Commun. Num. Theor. Phys. 08 (2014) 589 [arXiv: 1302.6445] [INSPIRE].

[34] F. Brown, Single-valued Motivic Periods and Multiple Zeta Values, SIGMA 2 (2014) e25 [arXiv: 1309.5309] [INSPIRE].

[35] F. Brown, Notes on motivic periods, Commun. Num. Theor. Phys. 11 (2015) 557 [arXiv:1512.06410].

[36] A. Beilinson, A. Varchenko, A. Goncharov and V. Shekhtman, Projective Geometry and K-theory, Leningrad Math. J. 3 (1991) 523.

[37] O. Schnetz, Graphical hyperlogarithms, talk within the trimester Periods in Number Theory, Algebraic Geometry and Physics, HIM, Bonn, Germany, Feb. 27, 2018.

[38] F. Brown, A class of non-holomorphic modular forms I, 2017, Res. Math. Sci. 5 (2018) [arXiv: 1707.01230] [INSPIRE].

[39] F. Brown, A class of non-holomorphic modular forms II: equivariant iterated Eisenstein integrals, arXiv: 1708.03354.

[40] Y.-t. Huang, O. Schlotterer and C. Wen, Universality in string interactions, JHEP 09 (2016) 155 [arXiv: 1602.01674] [inSPIRE].

[41] T. Azevedo, M. Chiodaroli, H. Johansson and O. Schlotterer, Heterotic and bosonic string amplitudes via field theory, JHEP 10 (2018) 012 [arXiv: 1803.05452] [INSPIRE]. 
[42] A.V. Kotikov, Differential equations method: New technique for massive Feynman diagrams calculation, Phys. Lett. B 254 (1991) 158 [INSPIRE].

[43] N. Arkani-Hamed, J.L. Bourjaily, F. Cachazo and J. Trnka, Local Integrals for Planar Scattering Amplitudes, JHEP 06 (2012) 125 [arXiv:1012.6032] [INSPIRE].

[44] J.M. Henn, Multiloop integrals in dimensional regularization made simple, Phys. Rev. Lett. 110 (2013) 251601 [arXiv: 1304.1806] [INSPIRE].

[45] L. Adams and S. Weinzierl, The $\varepsilon$-form of the differential equations for Feynman integrals in the elliptic case, Phys. Lett. B 781 (2018) 270 [arXiv:1802.05020] [InSPIRE].

[46] J. Broedel, C. Duhr, F. Dulat, B. Penante and L. Tancredi, Elliptic Feynman integrals and pure functions, arXiv: 1809.10698 [INSPIRE].

[47] S. Mizera, Combinatorics and Topology of Kawai-Lewellen-Tye Relations, JHEP 08 (2017) 097 [arXiv: 1706 . 08527] [INSPIRE].

[48] L. Kronecker, Zur Theorie der elliptischen Funktionen, Mathematische Werke IV (1881) 313.

[49] F. Brown and A. Levin, Multiple elliptic polylogarithms, arXiv:1110.6917.

[50] D. Zagier, Periods of modular forms and Jacobi theta functions, Invent. Math. 104 (1991) 449.

[51] D.J. Gross, J.A. Harvey, E.J. Martinec and R. Rohm, Heterotic String Theory. 2. The Interacting Heterotic String, Nucl. Phys. B 267 (1986) 75 [INSPIRE].

[52] N. Sakai and Y. Tanii, One Loop Amplitudes and Effective Action in Superstring Theories, Nucl. Phys. B 287 (1987) 457 [INSPIRE].

[53] J.R. Ellis, P. Jetzer and L. Mizrachi, One Loop String Corrections to the Effective Field Theory, Nucl. Phys. B 303 (1988) 1 [inSPIRE].

[54] M. Abe, H. Kubota and N. Sakai, Loop Corrections to the $E_{8} \times E_{8}$ Heterotic String Effective Lagrangian, Nucl. Phys. B 306 (1988) 405 [INSPIRE].

[55] M.B. Green, J.H. Schwarz and L. Brink, $N=4$ Yang-Mills and $N=8$ Supergravity as Limits of String Theories, Nucl. Phys. B 198 (1982) 474 [inSPIRE].

[56] N. Berkovits, Multiloop amplitudes and vanishing theorems using the pure spinor formalism for the superstring, JHEP 09 (2004) 047 [hep-th/0406055] [INSPIRE].

[57] E. D'Hoker and D.H. Phong, The Geometry of String Perturbation Theory, Rev. Mod. Phys. 60 (1988) 917 [INSPIRE].

[58] A.G. Tsuchiya, On new theta identities of fermion correlation functions on genus $g$ Riemann surfaces, arXiv:1710.00206 [INSPIRE].

[59] A.G. Tsuchiya, On the pole structures of the disconnected part of hyper elliptic $g$ loop $M$ point super string amplitudes, arXiv:1209.6117 [INSPIRE].

[60] C.M. Hull and P.K. Townsend, Unity of superstring dualities, Nucl. Phys. B 438 (1995) 109 [hep-th/9410167] [INSPIRE].

[61] M.B. Green and M. Gutperle, Effects of D instantons, Nucl. Phys. B 498 (1997) 195 [hep-th/9701093] [INSPIRE].

[62] M.B. Green, H.-h. Kwon and P. Vanhove, Two loops in eleven-dimensions, Phys. Rev. D 61 (2000) 104010 [hep-th/9910055] [INSPIRE]. 
[63] M.B. Green and P. Vanhove, Duality and higher derivative terms in M-theory, JHEP 01 (2006) 093 [hep-th/0510027] [INSPIRE].

[64] M.B. Green, S.D. Miller and P. Vanhove, $\mathrm{SL}(2, \mathbb{Z})$-invariance and D-instanton contributions to the $D^{6} R^{4}$ interaction, Commun. Num. Theor. Phys. 09 (2015) 307 [arXiv:1404.2192] [INSPIRE].

[65] B. Schoeneberg, Elliptic modular functions, Springer-Verlag, (1974).

[66] J.E. Gerken and J. Kaidi, Holomorphic subgraph reduction of higher-valence modular graph forms, arXiv: 1809.05122 [INSPIRE].

[67] J. Broedel, N. Matthes and O. Schlotterer, Relations between elliptic multiple zeta values and a special derivation algebra, J. Phys. A 49 (2016) 155203 [arXiv:1507.02254] [INSPIRE].

[68] D. Zagier and H. Gangl, Classical and elliptic polylogarithms and special values of L-series, in The arithmetic and geometry of algebraic cycles (Banff, AB, 1998), vol. 548 of NATO Sci. Ser. C Math. Phys. Sci., pp. 561-615, Kluwer Acad. Publ., Dordrecht, (2000).

[69] F. Brown, Multiple modular values and the relative completion of the fundamental group of $m_{1,1}$, arXiv: 1407.5167.

[70] F. Zerbini, Elliptic multiple zeta values, modular graph functions and genus 1 superstring scattering amplitudes, Ph.D. thesis, Bonn U., 2017. arXiv:1804.07989 [INSPIRE].

[71] D. Oprisa and S. Stieberger, Six gluon open superstring disk amplitude, multiple hypergeometric series and Euler-Zagier sums, hep-th/0509042 [INSPIRE].

[72] C.R. Mafra, O. Schlotterer and S. Stieberger, Complete N-Point Superstring Disk Amplitude II. Amplitude and Hypergeometric Function Structure, Nucl. Phys. B 873 (2013) 461 [arXiv: 1106.2646] [INSPIRE].

[73] O. Schlotterer, Amplitude relations in heterotic string theory and Einstein-Yang-Mills, JHEP 11 (2016) 074 [arXiv: 1608.00130] [INSPIRE].

[74] C.R. Mafra, O. Schlotterer and S. Stieberger, Complete N-Point Superstring Disk Amplitude I. Pure Spinor Computation, Nucl. Phys. B 873 (2013) 419 [arXiv:1106.2645] [InSPIRE].

[75] J. Broedel, O. Schlotterer and S. Stieberger, Polylogarithms, Multiple Zeta Values and Superstring Amplitudes, Fortsch. Phys. 61 (2013) 812 [arXiv:1304.7267] [INSPIRE].

[76] D. Zagier, The Rankin-Selberg method for automorphic functions which are not of rapid decay, J. Fac. Sci. Univ. Tokyo Sect. IA Math. 28 (1981) 415 (1982).

[77] C. Angelantonj, I. Florakis and B. Pioline, A new look at one-loop integrals in string theory, Commun. Num. Theor. Phys. 6 (2012) 159 [arXiv:1110.5318] [INSPIRE].

[78] W. Lerche, B.E.W. Nilsson and A.N. Schellekens, Heterotic String Loop Calculation of the Anomaly Cancelling Term, Nucl. Phys. B 289 (1987) 609 [INSPIRE].

[79] W. Lerche, Elliptic Index and Superstring Effective Actions, Nucl. Phys. B 308 (1988) 102 [INSPIRE].

[80] J.M. Drummond, P.J. Heslop, P.S. Howe and S.F. Kerstan, Integral invariants in $N=4$ SYM and the effective action for coincident D-branes, JHEP 08 (2003) 016 [hep-th/0305202] [INSPIRE].

[81] P.S. Howe, U. Lindström and L. Wulff, $D=10$ supersymmetric Yang-Mills theory at $\alpha^{\prime 4}$, JHEP 07 (2010) 028 [arXiv: 1004.3466] [INSPIRE]. 
[82] G. Bossard, P.S. Howe, U. Lindström, K.S. Stelle and L. Wulff, Integral invariants in maximally supersymmetric Yang-Mills theories, JHEP 05 (2011) 021 [arXiv:1012.3142] [INSPIRE].

[83] D.J. Gross and J.H. Sloan, The Quartic Effective Action for the Heterotic String, Nucl. Phys. B 291 (1987) 41 [INSPIRE].

[84] Y. Kikuchi and C. Marzban, Low-energy Effective Lagrangian of Heterotic String Theory, Phys. Rev. D 35 (1987) 1400 [inSPIRE].

[85] E.A. Bergshoeff and M. de Roo, The Quartic Effective Action of the Heterotic String and Supersymmetry, Nucl. Phys. B 328 (1989) 439 [INSPIRE].

[86] A.A. Tseytlin, Heterotic type-I superstring duality and low-energy effective actions, Nucl. Phys. B 467 (1996) 383 [hep-th/9512081] [INSPIRE].

[87] S. Stieberger and T.R. Taylor, NonAbelian Born-Infeld action and type-I. Heterotic duality (1): Heterotic F**6 terms at two loops, Nucl. Phys. B 647 (2002) 49 [hep-th/0207026] [INSPIRE].

[88] G. Bossard, C. Cosnier-Horeau and B. Pioline, Protected couplings and BPS dyons in half-maximal supersymmetric string vacua, Phys. Lett. B 765 (2017) 377 [arXiv: 1608.01660] [INSPIRE].

[89] G. Bossard, C. Cosnier-Horeau and B. Pioline, Four-derivative couplings and BPS dyons in heterotic CHL orbifolds, SciPost Phys. 3 (2017) 008 [arXiv:1702.01926] [INSPIRE].

[90] J. Polchinski and E. Witten, Evidence for heterotic - type-I string duality, Nucl. Phys. B 460 (1996) 525 [hep-th/9510169] [INSPIRE].

[91] C.R. Mafra and O. Schlotterer, One-loop superstring six-point amplitudes and anomalies in pure spinor superspace, JHEP 04 (2016) 148 [arXiv: 1603.04790] [INSPIRE].

[92] C.R. Mafra and O. Schlotterer, Double-Copy Structure of One-Loop Open-String Amplitudes, Phys. Rev. Lett. 121 (2018) 011601 [arXiv:1711.09104] [INSPIRE].

[93] S. Lee and O. Schlotterer, Fermionic one-loop amplitudes of the RNS superstring, JHEP 03 (2018) 190 [arXiv: 1710.07353] [InSPIRE].

[94] A. Tsuchiya, More on One Loop Massless Amplitudes of Superstring Theories, Phys. Rev. D 39 (1989) 1626 [INSPIRE].

[95] A. Gregori, E. Kiritsis, C. Kounnas, N.A. Obers, P.M. Petropoulos and B. Pioline, $R^{2}$ corrections and nonperturbative dualities of $N=4$ string ground states, Nucl. Phys. B 510 (1998) 423 [hep-th/9708062] [INSPIRE].

[96] N.E.J. Bjerrum-Bohr and P. Vanhove, Explicit Cancellation of Triangles in One-loop Gravity Amplitudes, JHEP 04 (2008) 065 [arXiv:0802.0868] [INSPIRE].

[97] D.M. Richards, The One-Loop Five-Graviton Amplitude and the Effective Action, JHEP 10 (2008) 042 [arXiv: 0807.2421] [INSPIRE].

[98] M.B. Green, C.R. Mafra and O. Schlotterer, Multiparticle one-loop amplitudes and S-duality in closed superstring theory, JHEP 10 (2013) 188 [arXiv:1307.3534] [INSPIRE].

[99] J.E. Gerken, A. Kleinschmidt, C. Mafra and O. Schlotterer, to appear.

[100] J. Polchinski, String theory. Vol. 1: An introduction to the bosonic string, Cambridge Monographs on Mathematical Physics. Cambridge University Press, (2007). 
[101] R. Blumenhagen, D. Lüst and S. Theisen, Basic concepts of string theory, Theoretical and Mathematical Physics, Springer, Heidelberg, Germany, (2013).

[102] C.R. Mafra and O. Schlotterer, Non-abelian Z-theory: Berends-Giele recursion for the $\alpha^{\prime}$-expansion of disk integrals, JHEP 01 (2017) 031 [arXiv: 1609.07078] [INSPIRE].

[103] S. Stieberger, Constraints on Tree-Level Higher Order Gravitational Couplings in Superstring Theory, Phys. Rev. Lett. 106 (2011) 111601 [arXiv:0910.0180] [INSPIRE].

[104] H. Kawai, D.C. Lewellen and S.H.H. Tye, A Relation Between Tree Amplitudes of Closed and Open Strings, Nucl. Phys. B 269 (1986) 1 [INSPIRE].

[105] N. Matthes, Elliptic multiple zeta values. Ph.D. Thesis, Universität Hamburg, Germany, (2016).

[106] S. Bloch, M. Kerr and P. Vanhove, A Feynman integral via higher normal functions, Compos. Math. 151 (2015) 2329 [arXiv:1406.2664] [INSPIRE].

[107] L. Adams and S. Weinzierl, Feynman integrals and iterated integrals of modular forms, Commun. Num. Theor. Phys. 12 (2018) 193 [arXiv:1704.08895] [INSPIRE].

[108] E. Remiddi and L. Tancredi, An Elliptic Generalization of Multiple Polylogarithms, Nucl. Phys. B 925 (2017) 212 [arXiv:1709.03622] [INSPIRE].

[109] J.L. Bourjaily, A.J. McLeod, M. Spradlin, M. von Hippel and M. Wilhelm, Elliptic Double-Box Integrals: Massless Scattering Amplitudes beyond Polylogarithms, Phys. Rev. Lett. 120 (2018) 121603 [arXiv:1712.02785] [INSPIRE].

[110] J. Broedel, C. Duhr, F. Dulat and L. Tancredi, Elliptic polylogarithms and iterated integrals on elliptic curves. Part I: general formalism, JHEP 05 (2018) 093 [arXiv:1712.07089] [INSPIRE].

[111] J. Broedel, C. Duhr, F. Dulat and L. Tancredi, Elliptic polylogarithms and iterated integrals on elliptic curves II: an application to the sunrise integral, Phys. Rev. D 97 (2018) 116009 [arXiv: 1712.07095] [INSPIRE].

[112] J. Broedel, C. Duhr, F. Dulat, B. Penante and L. Tancredi, Elliptic symbol calculus: from elliptic polylogarithms to iterated integrals of Eisenstein series, JHEP 08 (2018) 014 [arXiv: 1803.10256] [INSPIRE].

[113] L. Adams, E. Chaubey and S. Weinzierl, Planar Double Box Integral for Top Pair Production with a Closed Top Loop to all orders in the Dimensional Regularization Parameter, Phys. Rev. Lett. 121 (2018) 142001 [arXiv: 1804.11144] [INSPIRE].

[114] L. Adams, E. Chaubey and S. Weinzierl, Analytic results for the planar double box integral relevant to top-pair production with a closed top loop, JHEP 10 (2018) 206 [arXiv: 1806. 04981] [INSPIRE].

[115] J. Broedel, C. Duhr, F. Dulat, B. Penante and L. Tancredi, From modular forms to differential equations for Feynman integrals, in KMPB Conference: Elliptic Integrals, Elliptic Functions and Modular Forms in Quantum Field Theory Zeuthen, Germany, October 23-26, 2017, arXiv:1807.00842 [INSPIRE].

[116] J. Blümlein, A. De Freitas, M. Van Hoeij, E. Imamoglu, P. Marquard and C. Schneider, The $\rho$ parameter at three loops and elliptic integrals, PoS (LL2018) 017 (2018) [arXiv: 1807.05287] [INSPIRE]. 
[117] J. Blümlein, Iterative Non-iterative Integrals in Quantum Field Theory, in KMPB Conference: Elliptic Integrals, Elliptic Functions and Modular Forms in Quantum Field Theory Zeuthen, Germany, October 23-26, 2017, arXiv:1808.08128 [INSPIRE].

[118] J. Broedel and O. Schlotterer, One-loop string scattering amplitudes as iterated Eisenstein integrals, in KMPB Conference: Elliptic Integrals, Elliptic Functions and Modular Forms in Quantum Field Theory Zeuthen, Germany, October 23-26, 2017, 2018.

[119] F. Zerbini, Modular and holomorphic graph function from superstring amplitudes, in KMPB Conference: Elliptic Integrals, Elliptic Functions and Modular Forms in Quantum Field Theory Zeuthen, Germany, October 23-26, 2017, arXiv:1807.04506 [INSPIRE].

[120] J. Broedel, E. Panzer, O. Schlotterer and F. Zerbini, to appear.

[121] E. D'Hoker and M.B. Green, Zhang-Kawazumi Invariants and Superstring Amplitudes, J. Number Theory 144 (2014) 111 [arXiv: 1308.4597] [INSPIRE].

[122] E. D'Hoker, M.B. Green, B. Pioline and R. Russo, Matching the $D^{6} R^{4}$ interaction at two-loops, JHEP 01 (2015) 031 [arXiv: 1405.6226] [INSPIRE].

[123] E. D'Hoker, M.B. Green and B. Pioline, Higher genus modular graph functions, string invariants and their exact asymptotics, Commun. Math. Phys. (2018) [arXiv:1712.06135] [INSPIRE].

[124] E. D'Hoker, M.B. Green and B. Pioline, Asymptotics of the $D^{8} \mathcal{R}^{4}$ genus-two string invariant, arXiv:1806.02691 [INSPIRE].

[125] A. Weil, Elliptic functions according to Eisenstein and Kronecker, Classics in Mathematics, Springer-Verlag, Berlin, Germany, (1999). 